\title{
Aviate, Navigate: Functional Visualizations of Asymmetric Flight Envelope Limits
}

Supporting Pilot Decision Making in Failure Scenarios

A. D. T. Rijndorp

April 29, 2016
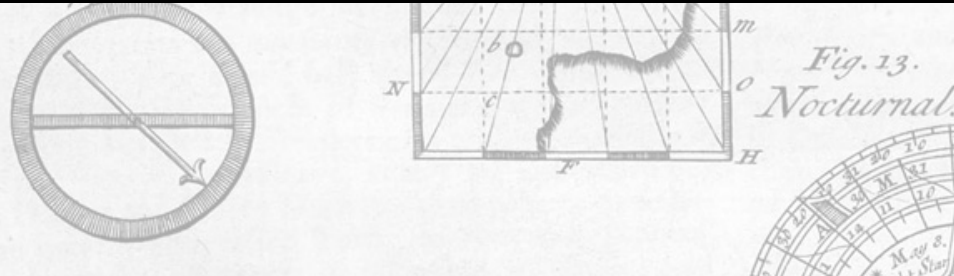



\title{
Aviate, Navigate: Functional Visualizations of Asymmetric Flight Envelope Limits \\ Supporting Pilot Decision Making in Failure Scenarios
}

\author{
Master of Science Thesis
}

For obtaining the degree of Master of Science in Aerospace Engineering at Delft University of Technology

A. D. T. Rijndorp

April 29, 2016 


\section{TUDelft}

Delft University of Technology

Copyright (c) A. D. T. Rijndorp

All rights reserved.

Cover page image: Table of Geography, Hydrography, and Navigation, from the 1728 "Cyclopaedia", Volume 1. 


\section{DelfT University Of TEChNOLOGY \\ DEPARTMENT OF \\ Control AND Simulation}

The undersigned hereby certify that they have read and recommend to the Faculty of Aerospace Engineering for acceptance a thesis entitled "Aviate, Navigate: Functional Visualizations of Asymmetric Flight Envelope Limits" by A. D. T. Rijndorp in partial fulfillment of the requirements for the degree of Master of Science.

Dated: April 29, 2016

Readers:

prof. dr. ir. M. Mulder

dr. ir. C. Borst

dr. ir. C. C. de Visser

ir. O. Stroosma

dr. A. Sharpanskykh 



\section{Preface}

In front of you lies the result of a study that was conducted over the past several months in order to meet the graduation qualifications for the graduate program of the faculty of Aerospace Engineering at Delft University of Technology, the Netherlands.

In the spring of 2015, I found myself wondering in what field I would want to conduct my research. I was already quite certain that I wanted to ask Clark to be my supervisor, as I've alsways had an interest in user experience and human psychology. Clark knows one or two things about that. At first, he suggested me to just drop by whenever I returned from my internship in the US. A day later though, he sent me a project outline for a topic requiring a creative mind, a multidisciplinary perspective, and simulation skills. I now realize that at that point, I didn't really have a choice anymore. I don't know if Clark was predetermined to have me take the assignment, but if he was, his approach worked.

So I took the assignment. Fast forward about a year, and this document is the result. The work revolves around the central question of how we might make the best use of a pilot's probem-solving skills in case of an emergency where the aircraft's motion capabilities are more restricted than usual, for instance due to wing damage. The approach taken here is to provide pilots with new information on the displays they already use. As systems like these require detailed information that we are currently not able to obtain in reality, this work is in a way a look into a not-so-near future where we do expect this information to become available.

I'd like to thank Clark, for always being available for a meeting, helping me in finding and maintaining a clear course of action, and providing excellent feedback on previous versions of this document. A really big thanks also goes out to Olaf for always asking the right question at the right time, and for the countless hours spent on supporting me in getting the experiment I conducted up and running. Coen has been very helpful, especially in making sure that any odd statements I would inadvertently make in my paper wouldn't make it to the final version and in providing valuable input during our meetings. I'd like to thank Max and René for suggesting ways to set up the experiment and for their feedback. Thanks also go to Martijn IJtsma for proof-reading the final version of the paper. Joost Ellerbroek deserves credit for providing the initial interface code. 
Finally, I'd like to thank all the pilots who participated in the experiment. It was a very rewarding experience to see them internalize concepts that a few months earlier only existed in my head, and their feedback has been extremely valuable.

Tom Rijndorp

Delft, the Netherlands

April 29, 2016 


\section{Acronyms}

$\begin{array}{ll}\text { AH } & \text { Abstraction Hierarchy } \\ \text { AMS } & \text { Amsterdam Schiphol Airport } \\ \text { APA } & \text { Automated Path Planning Aid } \\ \text { ATC } & \text { Air Traffic Control } \\ \text { C\&S } & \text { Control \& Simulation } \\ \text { CDU } & \text { Command Display Unit } \\ \text { DFDR } & \text { Digital Flight Data Recorder } \\ \text { DL } & \text { Decision Ladder } \\ \text { DOF } & \text { Degree of Freedom } \\ \text { DUT } & \text { Delft University of Technology } \\ \text { EFP } & \text { Emergency Flight Planner } \\ \text { EHSI } & \text { Electronic Horizontal Situation Indicator } \\ \text { EID } & \text { Ecological Interface Design } \\ \text { ELGS } & \text { Emergency Landing Guidance System } \\ \text { ELP } & \text { Emergency Landing Planner } \\ \text { FCS } & \text { Flight Control System } \\ \text { FD } & \text { Flight Director } \\ \text { FDD } & \text { Fault Detection and Diagnosis } \\ \text { FDI } & \text { Fault Detection and Isolation } \\ \text { FMS } & \text { Flight Management System } \\ \text { FTFC } & \text { Fault Tolerant Flight Control } \\ \text { GARTEUR } & \text { Group for Aeronautical Research and Technology in Europe } \\ \text { GPWS } & \text { Ground Proximity Warning System } \\ \text { ILS } & \text { Instrument Landing System } \\ \text { KBB } & \text { Knowledge-Based Behavior } \\ \text { MCP } & \text { Mode Control Panel } \\ \text { MPC } & \text { Model Predictive Control } \\ \end{array}$


NASA National Aeronautics and Space Administration

NASB Netherlands Aviation Safety Board

ND Navigation Display

NLR Netherlands Aerospace Laboratory

PFD Primary Flight Display

RBB Rule-Based Behavior

RECOVER GARTEUR Reconfigurable Control for Vehicle Emergency Return

SBB Skill-Based Behavior

SRK Skills, Rules, and Knowledge

SRS SIMONA Research Simulator

WDA Work Domain Analysis 


\section{List of Symbols}

\section{Greek Symbols}

$\begin{array}{ll}\alpha & \text { angle of attack } \\ \beta & \text { angle of side slip } \\ \delta_{a} & \text { aileron deflection } \\ \delta_{e} & \text { elevator deflection } \\ \delta_{r} & \text { rudder deflection } \\ \delta_{w} & \text { yoke (wheel) deflection } \\ \theta & \text { angle of pitch } \\ \tau & \text { time constant } \\ \phi & \text { angle of roll } \\ \psi & \text { heading angle }\end{array}$

\section{Roman Symbols}

$\begin{array}{ll}* & \text { trim state } \\ \gamma & \text { flight path angle } \\ g_{0} & \text { gravitational acceleration at Earth's surface } \\ H_{\text {in }}^{\text {out }} & \text { transfer function } \\ h & \text { altitude } \\ K & \text { gain } \\ n & \text { load factor } \\ p, q, r & \text { angular rate components in body frame } \\ \mathbb{T}_{a b} & \text { rotation of frame } a \text { with respect to } b\end{array}$


TAS true airspeed

$u, v, w \quad$ airspeed components in body frame

$V \quad$ airspeed

W weight 


\section{Contents}

Preface vi vi vis

Acronyms vii

$\begin{array}{ll}\text { List of Symbols } & \text { ix }\end{array}$

I Master of Science Thesis Paper 1

$\begin{array}{ll}\text { II Thesis Book of Appendices } & 21\end{array}$

$\begin{array}{ll}\text { A Literature Study } & 23\end{array}$

A-1 El Al flight 1862, a Case Study . . . . . . . . . . . . . . . . . . . . . . . . . . . . . . . . .

A-1-1 Sequence of Events . . . . . . . . . . . . . . . . . . . . 23

A-1-2 Failure Description . . . . . . . . . . . . . . . . . . . . . . . . . . . . . . . . . . . . . . . . . . . .

A-1-3 Decision Making . . . . . . . . . . . . . . . . . . . 25

A-2 Fault Tolerant Flight Control . . . . . . . . . . . . . . . . . . 27

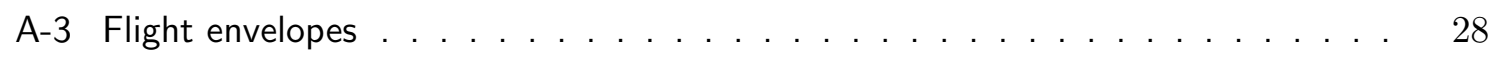

A-4 Ecological Interface Design . . . . . . . . . . . . . . . . . . . . . . 31

A-4-1 The Abstraction Hierarchy . . . . . . . . . . . . . . . . . 32

A-4-2 Skills, Rules, and Knowledge . . . . . . . . . . . . . . . . . 32

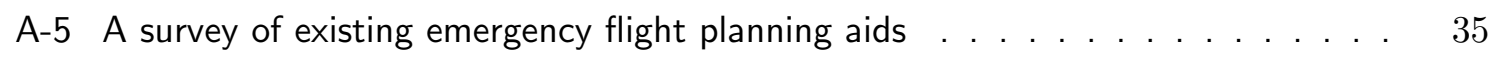

A-5-1 Emergency Flight Planner . . . . . . . . . . . . . . . . . . . . . 35

A-5-2 Emergency Landing Guidance System . . . . . . . . . . . . . . . . . . . . . . . . . . . . 37

A-5-3 Automated Path Planning Aid . . . . . . . . . . . . . . . . . 38

A-5-4 Emergency Landing Planner . . . . . . . . . . . . . . . . . 38

A-5-5 Discussion . . . . . . . . . . . . . . . . . . . . . . . 41 
B Model Properties 43

$\begin{array}{ll}\text { C DUECA Simulation Architecture } & 47\end{array}$

D RECOVER Trim Database Documentation $\quad \mathbf{5 1}$

D-1 Compatibility Notes . . . . . . . . . . . . . . . . . . . . . . . . . . 51

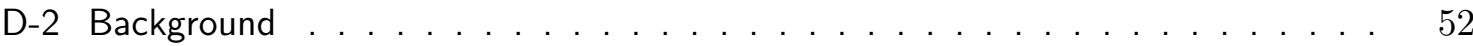

D-3 Non-interactive Automation . . . . . . . . . . . . . . . . . . . . . . 52

D-3-1 Function createTrimDatabase( $\ldots \ldots \ldots \ldots 5$

D-3-2 Class TrimDatabase ()$\ldots \ldots \ldots \ldots . \ldots \ldots$

D-3-3 Class TrimPoint . . . . . . . . . . . . . . . . . . . . 54

D-4 Known Issues with Trimming RECOVER . . . . . . . . . . . . . . . . . . 54

E Justification for a Single Pilot Operated Experiment 57

F Experiment Briefing $\quad 59$

G Questionnaire Results 63

$\begin{array}{ll}\text { H Latin Square Distribution } & 67\end{array}$

I Example Experiment Data Summary $\quad 69$

J Example TLX Sheet $\quad 71$

K Statistical Results $\quad 73$

K-1 Runway Selection . . . . . . . . . . . . . . . . . . . . . . . . . . . . 73

K-2 Intercept Strategy . . . . . . . . . . . . . . . . . . . . . . . . 74

K-3 Analysis of Work Load . . . . . . . . . . . . . . . . . . . . . 75

K-4 Analysis of Safety Metrics . . . . . . . . . . . . . . . . . . . 75

K-5 Analysis of Performance Metrics . . . . . . . . . . . . . . . . . . . . . 79 


\section{Part I}

\section{Master of Science Thesis Paper}





\title{
Aviate, Navigate: Functional Visualizations of Asymmetric Flight Envelope Limits
}

\author{
Tom Rijndorp*, Clark Borst ${ }^{\dagger}$, Coen de Visser ${ }^{\dagger}$, Olaf Stroosma ${ }^{\dagger}$, Max Mulder ${ }^{\dagger}$ and René van Paassen ${ }^{\dagger}$ \\ *Faculty of Aerospace Engineering, Delft University of Technology, Email: tomrijndorp@gmail.com \\ ${ }^{\dagger}$ Faculty of Aerospace Engineering, Delft University of Technology
}

\begin{abstract}
Current aircraft flight deck interfaces do not provide information on how a performance-altering failure constrains the aircraft's flight envelope. As a result, it is difficult for flight crews to plan an emergency landing trajectory. This study presents the results of the conceptual development of novel constraint-based interface symbology that aims to solve this issue.

As no fully functional on-line flight envelope prediction system implementations currently exist, a simplified dynamical model with an asymmetric flight envelope was developed to overcome this limitation. The proposed interface symbology integrates with the existing primary flight display and navigation display.

A human-in-the-loop experiment was conducted to validate the effectiveness of the used symbology. The results lend credibility to the belief that presenting flight envelope constraints in terms of reachable navigation states may improve short-term tactical planning and reduce the pilot's mental workload during emergency situations.
\end{abstract}

\section{INTRODUCTION}

Today, the task of flying an aircraft is predominately done by means of interacting with the on-board automated systems or autopilot. In certain events such as engine failures, control surface failures, or structural damage, pilots will need to reevaluate the conditions under which they are operating. They may need to determine a new mission objective and adjust their control strategy accordingly. The pilot's mantra here is "aviate, navigate, communicate", signifying both the tasks that need to be performed as well as their prioritization. That is, first, make sure that the aircraft regains and maintains stable flight. Then, derive a strategy to reach the (newly formulated) destination state, and only then inform other stakeholders of the issue at hand.

Performance-altering events cause changes in the safe flight envelope, making maneuvers that were safe to execute with a nominal flight envelope, dangerous or even impossible. This complicates a flight crew's ability to both aviate and navigate. A direct result of changes in the safe flight envelope is that the flight crew may become unaware of the limits of the now more constrained flight envelope. This poses challenges in terms of maintaining control of the aircraft, but most definitely also in terms of understanding implications on higher level goals such as replanning and landing.

When control is regained after an initial upset, the issue that remains is finishing the mission in an as safe as possible manner. However, being able to fly an aircraft does not necessarily imply that one is able to successfully navigate an aircraft to a destination state as well. In the presence of performancealtering failures, it is an open question how to solve the process of developing and executing a safe emergency navigation strategy to a suitable landing area.

Existing research contains proposals for fully automated emergency replanning and landing systems [1], planner modules that involve the pilot to select the best candidate from a set of trajectories defined by the automation [2], [3], [4], [5], [6], as well as interactive planners that require the pilot to interact with the planner module by iteratively adjusting and reviewing a landing plan [7].

With many automated systems, it is the case that the automation alleviates a task, but increases the total system complexity [8]. Increased complexity typically reduces the operator's overall understanding of the system [9]. This limits an operator's ability to take action should an unanticipated event occur. This also holds true for emergency landing planning automation: every case is unique to some degree and likely to cause a series of events that may not have been anticipated when designing the automation. Their innate ability to deal with unanticipated events is one of the primary reasons why human pilots are still required to be present in aircraft. The bottom line is that as long as human pilots are ultimately held responsible for the safety of all parties involved, they should have the authority - and access to information - to meet this demand in a meaningful way.

For this reason, it was found that a need exists for a graphical human-machine interface that can convey flight envelope estimation data in a functional manner, i.e., in such a way that it relates to the aviate and navigate functions of air transportation. More specifically, this interface should aid pilots in conceiving an ad-hoc navigation strategy in the presence of a performance-altering failure that resulted in an asymmetric maneuvering envelope. It is hypothesized that a functional representation of this information will result in improved pilot decision making while at the same time reducing the mental workload. This hypothesis was tested in a human-in-the-loop experiment, the results of which are also presented in this paper.

This paper is structured as follows: Section II describes the theoretical motivation for this study. Section III presents the dynamical model that was used along with its design considerations, and Section IV describes the interface development process. Section V comments on the human-in-theloop experiment that was conducted and Section VI describes the experiment's results. Discussion, recommendations, and conclusion sections complete this paper. 


\section{TheORETICAL Motivation}

\section{A. Flight 1862}

This research is motivated and inspired by El Al Flight 1862 (Flight 1862), a Boeing 747-200 freighter flight where the two starboard engines detached from the wing shortly after takeoff. The separated engines also inflicted damage upon the starboard wing's leading edge and hydraulics systems, rendering multiple control surfaces (among which the outboard ailerons) inoperable. A schematic overview of the failure mode is shown in Figure 1.

The original investigation mentioned that at higher angles of attack, the difference in lift between the left and right wing would increase as a result of the right wing leading edge damage. Therefore, increasing the angle of attack would generate a right wing down rolling moment [11].

At the final stage of the flight, the angle of attack was increased, most likely in order to reduce the descent rate. This led to a further increase in drag on top of the higher than nominal drag caused by the sideslip angle and an additional decrease in speed. This probably caused the pilots to increase the engine thrust. This series of events led to a large right wing down rolling moment due to the asymmetric lift distribution, the asymmetric thrust, and reduced right hand inboard aileron efficiency. At this point, control of the aircraft was lost [11].

Inspection of Flight 1862's Digital Flight Data Recorder (DFDR) data indeed revealed that yoke deflections of up to 60 degrees out of 80 degrees were required in order to maintain straight flight [12]. As a result of the aileron deflection limits, roll rate performance suffered.

An independent investigation by the Netherlands Aerospace Laboratory (NLR) and Delft University of Technology (TU Delft) revealed that, in hindsight, control could have been maintained by maintaining a higher airspeed, resulting in more control authority and higher maneuverability [12].

While Flight 1862's failure mode posed a significant challenge for the flight crew in terms of maintaining control, the failure also heavily affected the aircraft's maneuvering and navigation capabilities. The flight crew had no other means for developing an understanding of these capabilities other than observing the aircraft's response to their control inputs. In fact, one of the decisions that stood out was that the crew decided to land on runway 27 with a tail/crosswind approach instead of the runway in use, runway 06 . Runway 06 offered a headwind approach and was likely easier to navigate to.

We may never be able to understand all the factors in the decision making process of the flight crew, and therefore whether or not the best possible decision was made in terms of runway selection and navigation planning. However, it is a fact that the amount of information available to the flight crew was limited, both in terms of diagnostics as well as in terms of functionality. That is, no explicit information was available to the flight crew on how the aviate and navigate functions were affected by the failure. And when the outcome of an action is uncertain, it is difficult to plan ahead. It is therefore imaginable that providing pilots with a means to understand an aircraft's limited capabilities from this functional perspective may improve the tactical planning process.

\section{B. Developments in Enabling Technology}

In order to develop a system that presents information on attainable (navigation) states, knowledge of the flight envelope is required. Unfortunately, estimating the flight envelope of damaged aircraft is far from trivial. While much research is currently being done on Fault Detection and Isolation (FDI) and post-failure flight envelope estimation [13], [14], [15], [16], [17], [18], [19], [20], [21], [22], fully functioning online systems do not yet exist. One reason for this lack of existing implementations are the high dimensionality of the state space and the corresponding computational complexity. A second, more fundamental reason, is that all reachability based envelope prediction methods require an accurate global aerodynamic model, which in general is not available after a failure event. A promising solution to this issue in the form of a database driven envelope prediction method was recently introduced by Zhang et al. [22].

The primary driver for research in flight envelope prediction and estimation is the field of Fault Tolerant Flight Control (FTFC). The main goal of FTFC is to improve the controllability of aircraft after a system fault has occurred or damage has been sustained.

Fault tolerant control systems are, to some extent, a doubleedged sword. As these systems simplify the control task, potentially by seamlessly blending different control effector inputs in such a way that aircraft control is natural and predictable [23], it will be harder for a pilot to observe the amount of remaining control authority and thereby how safe a certain state or maneuver actually is. For this reason, flight envelope prediction is an important field of study as it enables Flight Envelope Protection (FEP) under off-nominal flight conditions, preventing pilots from operating outside the more constrained safe flight envelope.

However, even with a fault-tolerant, flight envelopeprotected control system but without an understanding what the off-nominal flight envelope implies in terms of maneuvering and navigation capabilities, pilots may still not be able to formulate a safe navigation strategy with a damaged aircraft.

\section{MODEL DEVELOPMENT}

For the purpose of performing an experiment, a dynamical aircraft model with an off-nominal flight envelope is required. Additionally, the model's flight envelope should be known. It was mentioned that fully functioning flight envelope prediction systems do not yet exist. Lastly, if the resulting aircraft dynamics are difficult to control, a fault-tolerant control system may have to be added: in a study on emergency landing planning by Meuleau et al., it was found that adding a faulttolerant controller was a prerequisite for effective use of the planner interface, as fault-tolerant controllers can alleviate the control task substantially [6].

In order to cope with this complexity in a way that would allow this study to be achievable both with present-day tech- 


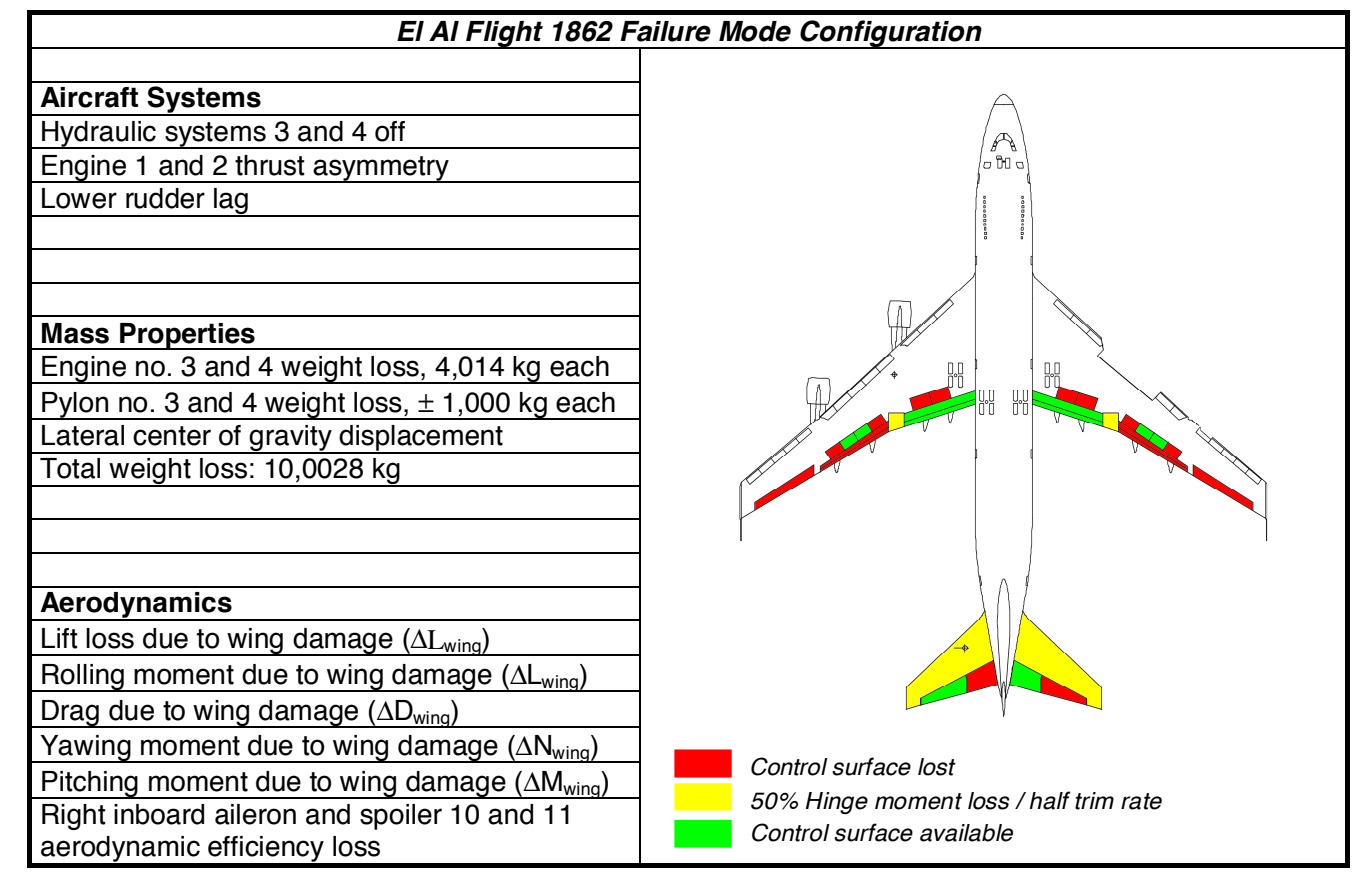

Fig. 1. El Al Flight 1862 failure mode [10]

nology and within the allocated time, a simplified model was developed.

Figure 2 shows the control loop of a hypothesized future aircraft system including a flight envelope estimation module, fault tolerant control system, the pilot, and the (graphical) interface. We can observe that the pilot is interacting with the Flight Control System (FCS), which in this case is assumed to be fault-tolerant. The fault or failure that alters the flight envelope of the aircraft also impairs the pilot's trained ability to estimate reachable aircraft states. To this end, a Fault Detection and Diagnosis (FDD) module coupled with a flight envelope estimation module may assist the pilot in interpreting the new, off-nominal flight envelope when this information is presented on the Electronic Flight Instrument System (EFIS) screens.

As it is generally not possible for a pilot to discriminate between performance limitations imposed by the FCS and performance limitations resulting from the aircraft's dynamics, the approach chosen in this study is to limit model complexity by lumping together the flight envelope estimation, FDD, FCS, and dynamics blocks, resulting in a simplified plant that can output data to the interface and accept pilot commands. This simplified, combined system which accepts a reference input, integrates the aircraft's dynamics and returns flight envelope data is depicted as 'Combined system' in Figure 2.

While vertical navigation planning is relevant to this field of research, as a first step, the focus of this study is on the lateral navigation capabilities and the control strategies pilots develop as a result of the remaining performance. As such, a simplified linear model does not need to consider vertical motion.
To constrain the flight envelope, a consequence of Flight 1862's wing damage was considered. An asymmetric lift distribution requires a compensating aileron deflection in order to maintain equilibrium. This results in a non-zero aileron deflection to maintain straight flight.

A model with these properties was obtained, starting from the GARTEUR RECOVER Boeing 747 model developed by Smaili et al. [25]. First, the model was trimmed for straight and level flight for a given flight condition and aircraft configuration. Next, the model was linearized, resulting in a set of continuous-time state space matrices. Then, new system and input matrices were formed using only the asymmetric states $p, r, \beta$, and $\phi$ and the aileron input $\delta_{a}$ [26]. Assuming the presence of an effective yaw damper and turn coordination system in the FCS, the system can be further simplified to the extent that only the roll subsidence eigenmode is retained, resulting in a simple transfer function that describes the roll behavior of the aircraft [27].

The model structure can be seen in Equation (1). The parameters $K_{p}$ and $\tau_{p}$ depend on the airspeed $V$. Speed-dependent values for these parameters can be derived by performing the procedure outlined here for multiple trim points.

$$
\begin{aligned}
\dot{p}(t) & =-\frac{1}{\tau_{p}(V)} p(t)+\frac{K_{p}(V)}{\tau_{p}(V)} \delta_{a}(t) \\
\dot{\phi}(t) & =p(t) \\
\dot{\psi}(t) & =\frac{g_{0}}{V(t)} \phi(t) \\
\dot{x}^{E}(t) & =V(t) \cos \gamma \cos \psi(t) \\
\dot{y}^{E}(t) & =V(t) \cos \gamma \sin \psi(t)
\end{aligned}
$$

With this result, the severity of the failure can be modeled by selecting an aileron or yoke deflection required to maintain a 


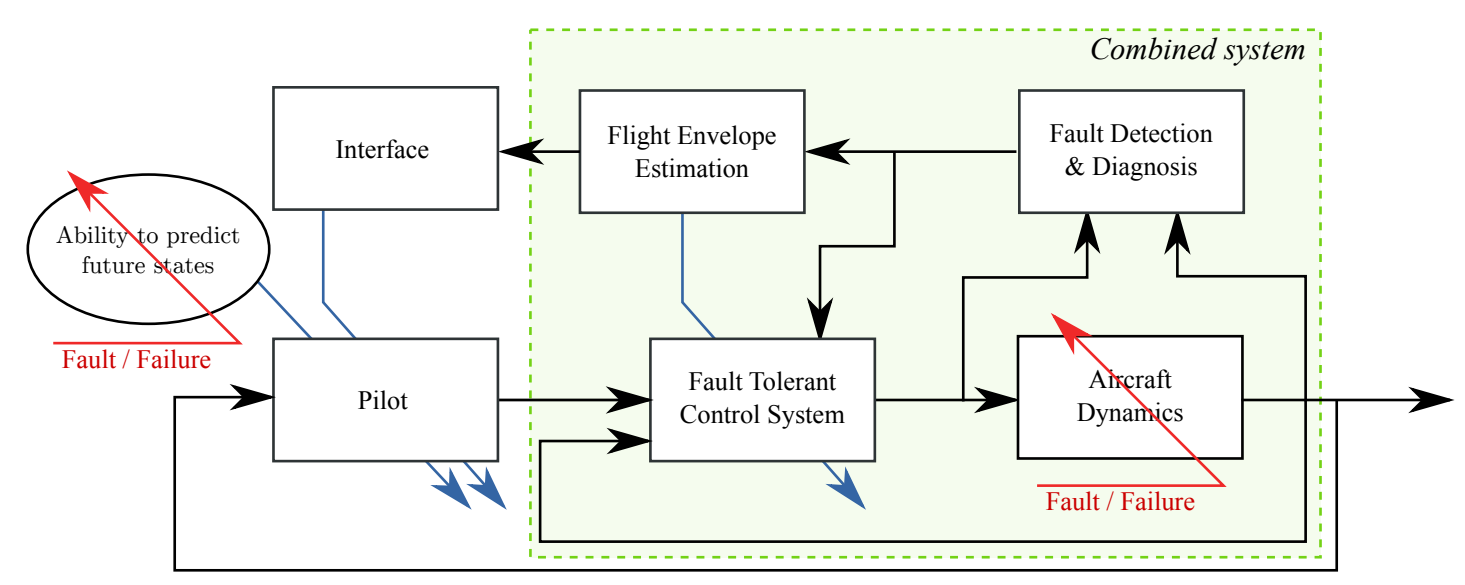

Fig. 2. The control loop including pilot and interface (adapted from [24])

zero roll rate. In order to control the aircraft, this value should be chosen in between the control effector's saturation limits. The larger the magnitude of this value, the more asymmetric the roll behavior of the aircraft will be.

Now, two approaches can be taken to modify the navigation performance resulting from this simplified model:

1) We can increase the airspeed, thereby reducing the steady-state aileron deflection resulting from the lower angle of attack. This would represent the same damage case at a different airspeed.

2) We can keep the airspeed constant but alter the required aileron deflection. This would represent a different damage case at the same airspeed.

Regardless of the approach taken, the result is that the larger the steady-state aileron offset gets, the more asymmetric the aileron authority becomes. This results in lower attainable roll rates in one direction, and higher rates in the other. The resulting lateral maneuvering envelope is shown conceptually in Figure 3. For this study, the shaded area of safe combinations of roll rate and roll angle can be considered the safe flight envelope. This figure displays the set of safe roll rates given the current roll angle. As clockwise (positive) roll rates move away from the left roll angle limit, these rate/angle combinations are safe, as are negative rates at positive angles. In the right top corner (marked (1)), it can be seen that there are combinations of roll rate and roll angle that cause the roll angle limit to be exceeded. These combinations should be avoided. Equivalently, the same holds for the combinations in the opposite corner (marked (2)). However, because these attainable rates are lower and the opposite aileron authority is higher, these rates can be controlled back to zero faster.

Lastly, we may trim the controls by offsetting the stick input with the stick input that yields a zero roll rate, resulting in an interface that effectively commands the roll rate.

The result from Figure 3 mainly explains how wing damage may impair the lateral maneuvering capabilities. However, it does not yet clarify how reduced maneuvering capabilities become relevant to the higher level goal of navigating an aircraft. To this end, Figure 4 is introduced. This figure shows

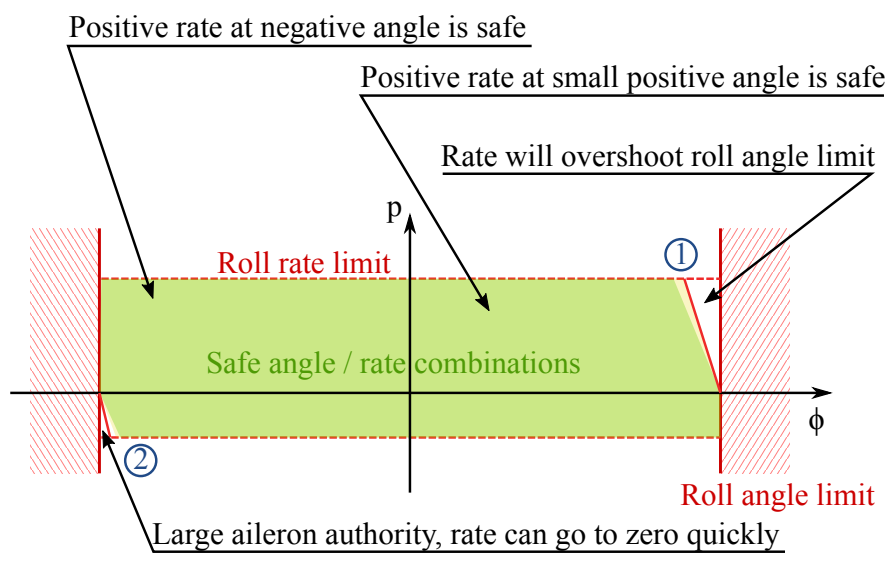

Fig. 3. Relation between safe roll rates and roll angles at a given airspeed for a simplified model with asymmetric aileron authority

the relation between the 'aviate' and 'navigate' tasks from the perspective of the state variables, while also making a connection to the temporal aspects.

The middle part of the figure articulates that the distinction between the two concepts is mainly one of time: we think of maneuvering as something that takes place on shorter time scales (seconds to minutes) and therefore is more dynamic. Navigating on the other hand, is more strategic and involves planning in the order of minutes and hours while mainly flying approximately straight paths.

The bottom part of the figure shows the aircraft states as they relate to one another in a first order approximation, i.e., this representation is most likely similar to a pilot's mental model of lateral aircraft dynamics. This model is also similar to the simplified model that was introduced here. The lower (less integrated) states represent the faster dynamics that relate to maneuvering, whereas the more integrated states such as position and heading relate to navigation, a higher order of abstraction representation of air transportation.

Finally, the top part of the figure tries to explain why system faults and failures like those experienced by Flight 1862 and other flights with wing damage and/or aileron failures are hard 


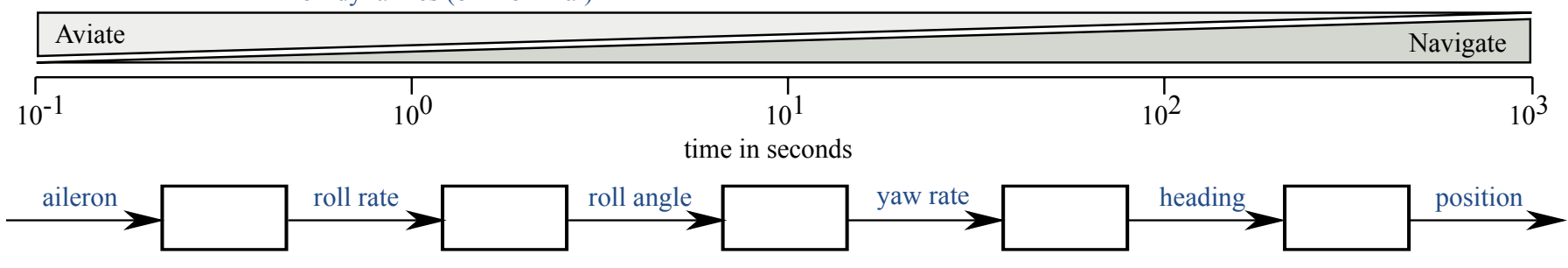

Fig. 4. Relation between maneuvering and navigation (simplified)

to deal with: as the (roll) dynamics become more constrained, the distinction between flying and navigating becomes even more ambiguous and navigating the aircraft to a goal state requires more anticipation than usual.

\section{INTERFACE DEVELOPMENT}

As the goal of the interface symbology presented in this work is to provide pilots with an understanding of the flight envelope, like the flight envelope itself, most of the interface elements provide a constraint-based representation. Interfaces that display a constraint-based representation of the work domain (or ecology) were formalized by Rasmussen and Vicente, under the term Ecological Interface Design (EID) [28]. EID is a design framework that was specifically developed to design interfaces that support operators in complex work domains, where unanticipated events may occur and the ideal control strategy may not be known in advance. While EID originally emerged in the field of nuclear process control, the framework has also been adapted to support pilots [29], [30], [31] and air traffic controllers [32]. The interfaces presented here have been inspired by principles from EID as well.

\section{A. Symbology}

Interface elements were added to both the (Boeing-style) Primary Flight Display (PFD) and Navigation Display (ND). These elements can be seen in Figure 5, labeled (1) through (5).

1) Attainable roll rates: The attainable roll rates (shown asymmetric in the figure due to the presence of the failure) are shown (see (1)). These were visualized as a shaded bar on the bank angle scale, with the vertical edges of the bar indicating the maximum steady-state roll rate presented as a five-second bank angle preview. This preview time was chosen to provide an appropriate scaling factor given the range of values that were to be used in the experiment. In this example, the bar covers an area of 30 degrees to the right, implying that the maximum steady-state roll rate is six degrees per second. The attainable roll rates can be derived from Equation (1) by substituting the aileron deflection limits, as is done in Equation (2).

$$
\begin{aligned}
& p_{\min }=K_{p} \delta_{a_{\max }} \\
& p_{\max }=K_{p} \delta_{a_{\min }}
\end{aligned}
$$

The main motivation for including this feature was that it provides a means to directly perceive the failure mode in terms of the roll dynamics. In the asymmetric case presented here, it is directly visible that performance will indeed be asymmetric and how the two attainable roll rate limits relate to one another, e.g. a ratio of approximately 6 to 1 in the example provided here. It is clear which turn can be initiated faster, which is useful for evasive maneuvers, and it gives a first impression on whether leveling out of a turn will be relatively fast or relatively slow.

2) Current roll rate: The arrow inside the shaded bar presented the current roll rate, again presented as a five-second angle preview (see (2)). In the figure, the arrow covers almost the entire shaded bar, indicating that the aircraft is currently rolling at almost its maximum rate. The difference between the current roll rate and the maximum rate indicates how much more rolling performance is available, which will be attained over time if the maximum control input is delivered.

3) Roll angle preview: The shaded triangles (see (3)) indicate the reachable set of roll angles within the next ten seconds. Again, this time interval was chosen to provide a good resolution given the dynamics of the model. This is done by calculating the largest roll angle that can be obtained by exerting a continuous maximum control input, initiating either a rolling motion either to the left or to the right. The indicators remain stationary if the calculated angle would be larger than the roll angle limit. This is the case in Figure 5 for the right bank limit (shown on the left of the PFD). Alternatively, after ten seconds a roll angle of 10 degrees left can be attained (shown on the right). This symbol was included to provide an alternative representation to the attainable roll rate limits to see whether pilots would have a clear preference for one symbol or the other: the attainable rate limits are stationary on the PFD screen (attached to the attitude scale), whereas the triangles rotate along with the attitude indicator (and the horizon). Additionally with this symbol a positive (right) bank attitude is represented as a negative (left) deviation.

4) Roll-out indicator: The Roll-out indicator (ROI) (see (4)) presented information on the angle covered in order to bring the current roll rate to zero. In the figure, the current bank angle is zero degrees. The ROI deviates approximately twelve degrees from the current bank angle, implying that - even given the maximum opposite stick input - the aircraft would roll out to twelve degrees of bank. Making the connection 

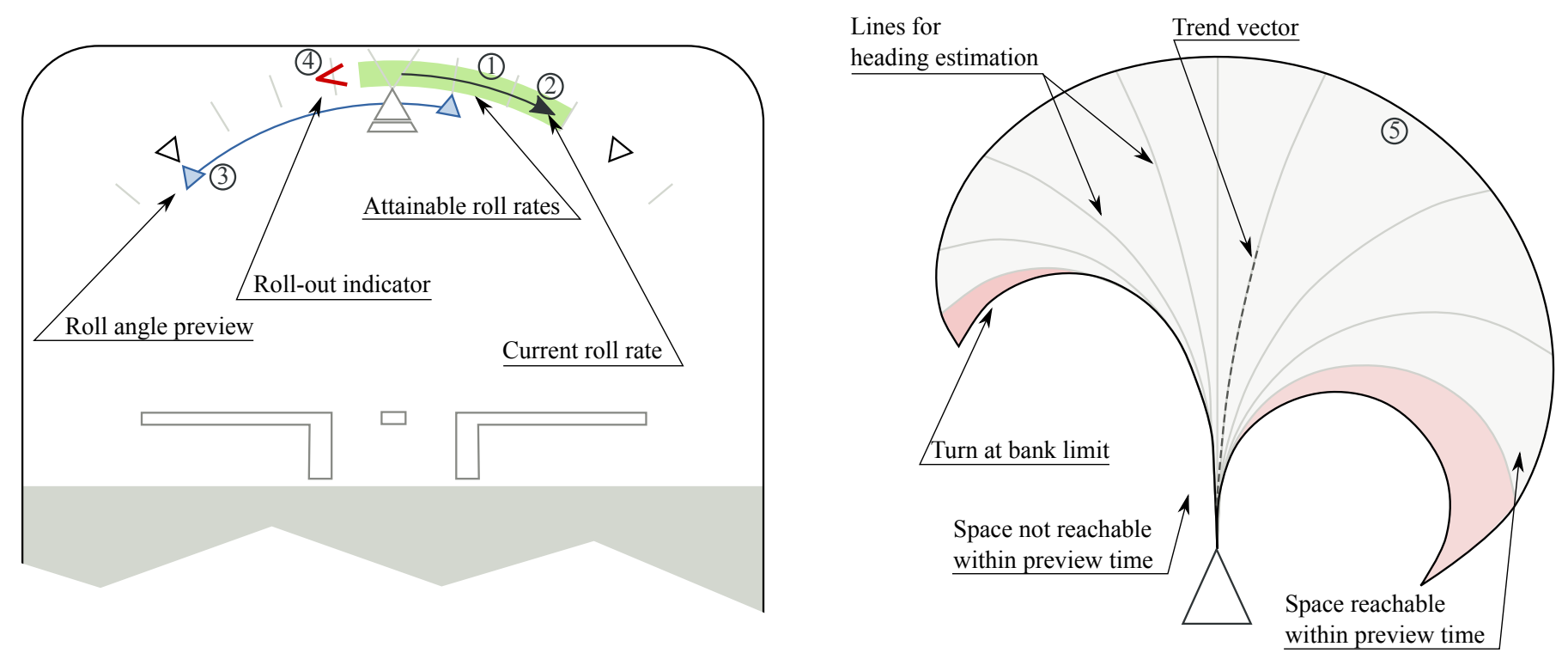

Fig. 5. Interface modifications on the PFD (left) and ND (right)

to Figure 3, we may realize that when the current state of the aircraft is at (1), the ROI would be located at the right roll angle limit (which is located on the left in a Boeing PFD). This symbol was included because it was hypothesized that the asymmetric rate performance would make it harder to not exceed the bank limits as was clarified in Figure 3. In a (potentially autopilot-controlled) flight control system without FEP, this symbol aids the pilot in staying within the maneuvering envelope. In a system with FEP, this symbol would explain why the control system is reducing the roll rate even though the bank limit is not yet attained.

5) Additional ND symbology: The ND was augmented with one additional symbol: the Navigation Envelope (NE) (see (5) in Figure 5). This symbol provided a sixty-second preview of the reachable navigational space, given the current dynamics and limits. The preview time was selected based on a tradeoff between introducing too much clutter (the NE will wrap back onto itself with a large preview time) and the zoom range that was expected to be used in the experiment. The ownship symbol and standard position trend vector are included in the figure to clarify where the NE would appear. Inside the shaded area, curves were included in order to facilitate heading estimation: the pilot can estimate the heading corresponding to any position by mentally interpolating in between the curves.

This is shown in Figure 6: if a pilot would want to intercept the vector shown in the figure, the NE explicates the control actions that can be employed to achieve this goal. In order to define a successful procedure, a pilot would mentally slide the vector down towards the ownship symbol. In this figure, the vector would then first become tangential to curve 1 at the point indicated. This means that a pilot could initiate a turn, i.e., position the trend vector on curve 1 , when the vector would touch curve 1 and intercept the vector at point $\mathrm{A}$. The same is true for curve 2 and point $\mathrm{B}$, or curve 3 and point $\mathrm{C}$, respectively. It should be noted that these trajectories would require an increasingly larger maximum bank angle. In fact, in order to intercept the vector at point $\mathrm{C}$ it can be seen that a turn at the maximum bank angle would be required. If no action would be taken and the vector would end up in the position indicated with 4 , it would be clear that an attempted intercept will cause overshooting the vector. This could also signify the need to pursue a different approach strategy altogether.

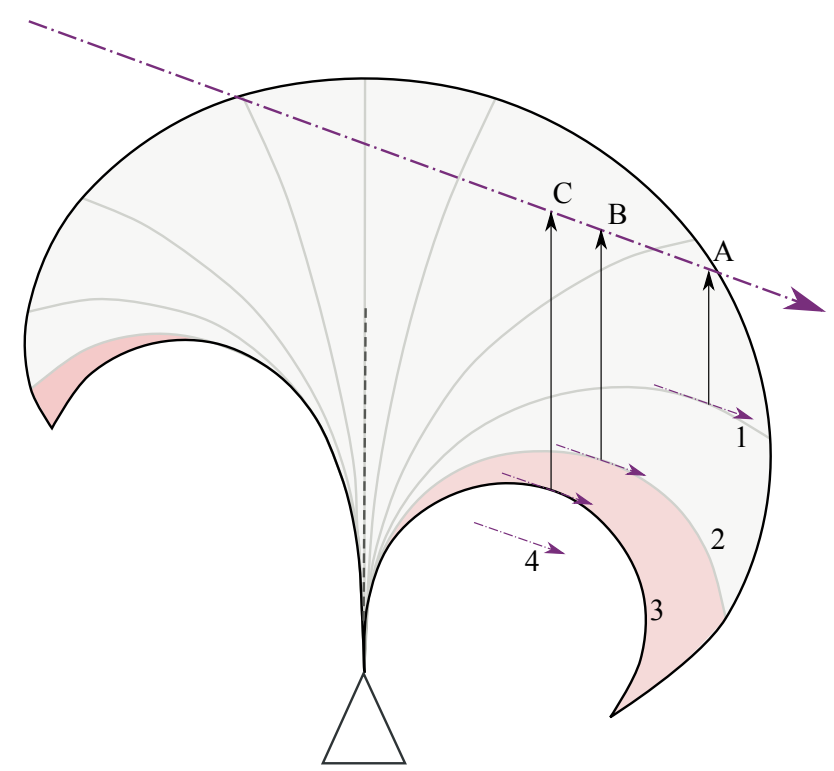

Fig. 6. Estimating different turns that will intercept the given vector

It should be noted that the curves in the figure do not represent turns at a given bank angle; instead, they represent turns at a given target bank angle. That is, using the dynamical model, a control sequence to go from the current bank angle to the target bank angle is calculated using a doublet input 


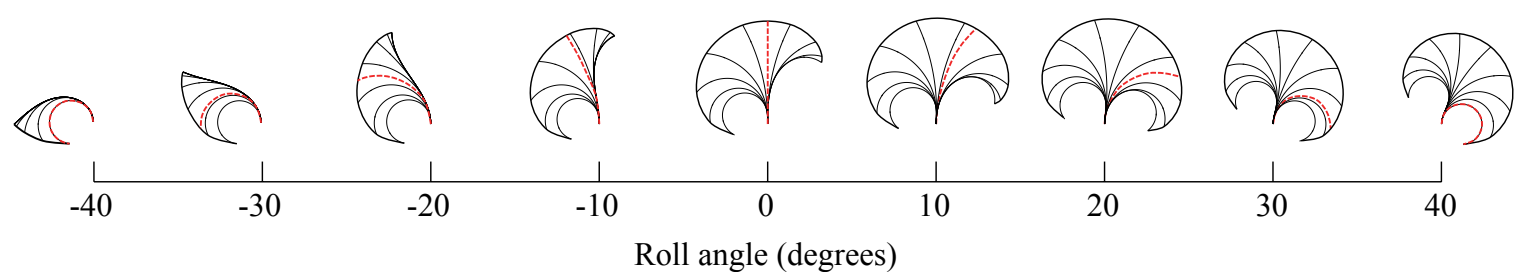

Fig. 7. Different NE shapes indicating how the reachable states are affected by the bank angle (asymmetric roll performance). The red dashed lines indicate the trajectory that will be flown given the current state.

with the maximum aileron authority. If the target bank angle is reached within the preview time, the remaining part of the calculated turn is performed at the target bank angle. Figure 7 shows what the NE may look like given different initial bank angles and an asymmetric aileron failure.

\section{B. Expected Interface Usage}

It was mentioned that the goal of the novel interface symbology is to allow pilots to understand and act upon cues that indicate off-nominal performance in an effective manner. Rasmussen's taxonomy of levels of cognitive control [33] may shed a light on how the interface symbology aims to accommodate this.

Rasmussen states that a distinction can be made between three levels of cognitive control: Skill-Based Behavior (SBB), Rule-Based Behavior (RBB), and Knowledge-Based Behavior (KBB). Operators employ SBB for simple, familiar tasks that require little to no mental effort, e.g., the simple tracking task of keeping an aircraft's wings level while flying in mild turbulence. RBB is employed when familiar solutions exist to familiar problems, e.g., flying a standard rate turn. KBB is triggered when neither SBB nor RBB can solve a problem. For instance, when a standard rate turn can not be properly executed because the desired rate can not be attained, a pilot will start reasoning about what may have happened to the aircraft that is causing this observation: the turn indicator could be broken, or there may be something wrong with the flight controls.

Which levels of behavior are utilized to perform a certain task ultimately depends on a number of factors, such as the operator's training and familiarity with the given situation, as well as the available support for the task, e.g., the interface.

It is expected that both the additional PFD and ND symbology can lower the required cognitive demand by allowing for "shortcuts" in the decision process. This may enable pilots to accelerate the decision making process, sometimes by employing RBB instead of KBB for elements of the navigation task. Figure 8 maps the processes and knowledge states relevant to a localizer intercept task in the presence of a performance-altering failure onto Rasmussen's Decision Ladder template. In the figure, we can observe processes and knowledge states associated with SBB, RBB, and KBB. These are annotated with the tasks and states for the intercept task.

The figure shows how the additional PFD symbology may enable direct perception of the parameters of the failure. This would happen primarily through feature (1) in Figure 5, as this symbol is invariant to changes in the bank angle and has a constant size for a given airspeed. Performing a localizer intercept will still require passing through a knowledge-based evaluation cycle.

$\mathrm{KBB}$ will still be required when using the NE for planning the initial trajectory, as full trajectories leading to the localizer are not visualized. However, with progressively smaller distances to the localizer, the NE will provide increasingly more cues as to which control action to perform, up to the point where there is only the possibility of immediately giving a control input to intercept the localizer. At these last stages, no $\mathrm{KBB}$ is required for factoring the failure information into the tactical planning and mentally integrating future states. The visited elements of the decision ladder for these different cases are shown in the lower part of the figure.

\section{EXPERIMENT}

A pilot's ability to reason with the developed interface symbology should be the definitive means of indicating the feasibility of any flight deck interface. Therefore, a human-inthe-loop experiment was conducted to assess the ability of the interfaces to improve pilot performance and decision making.

\section{A. Participants}

Nine pilots with various degrees of professional experience participated in this experiment. Their qualifications are shown in Table I. All pilots had prior experience with glass-cockpit displays.

TABLE I

PILOT QUALIFICATIONS

\begin{tabular}{ll}
\hline \hline License type & Amount \\
\hline PPL & $1^{\dagger}$ \\
CPL & 1 \\
ATPL & $7^{*}$ \\
\hline
\end{tabular}

$\dagger$ This participant had no prior experience with the ND position trend vector

* One participant was still in training with four months remaining, three participants had experience as research test pilot.

\section{B. Procedure}

A high-level overview of the procedure each participant engaged in is shown in Table II. The elements of the procedure are elaborated upon below. 


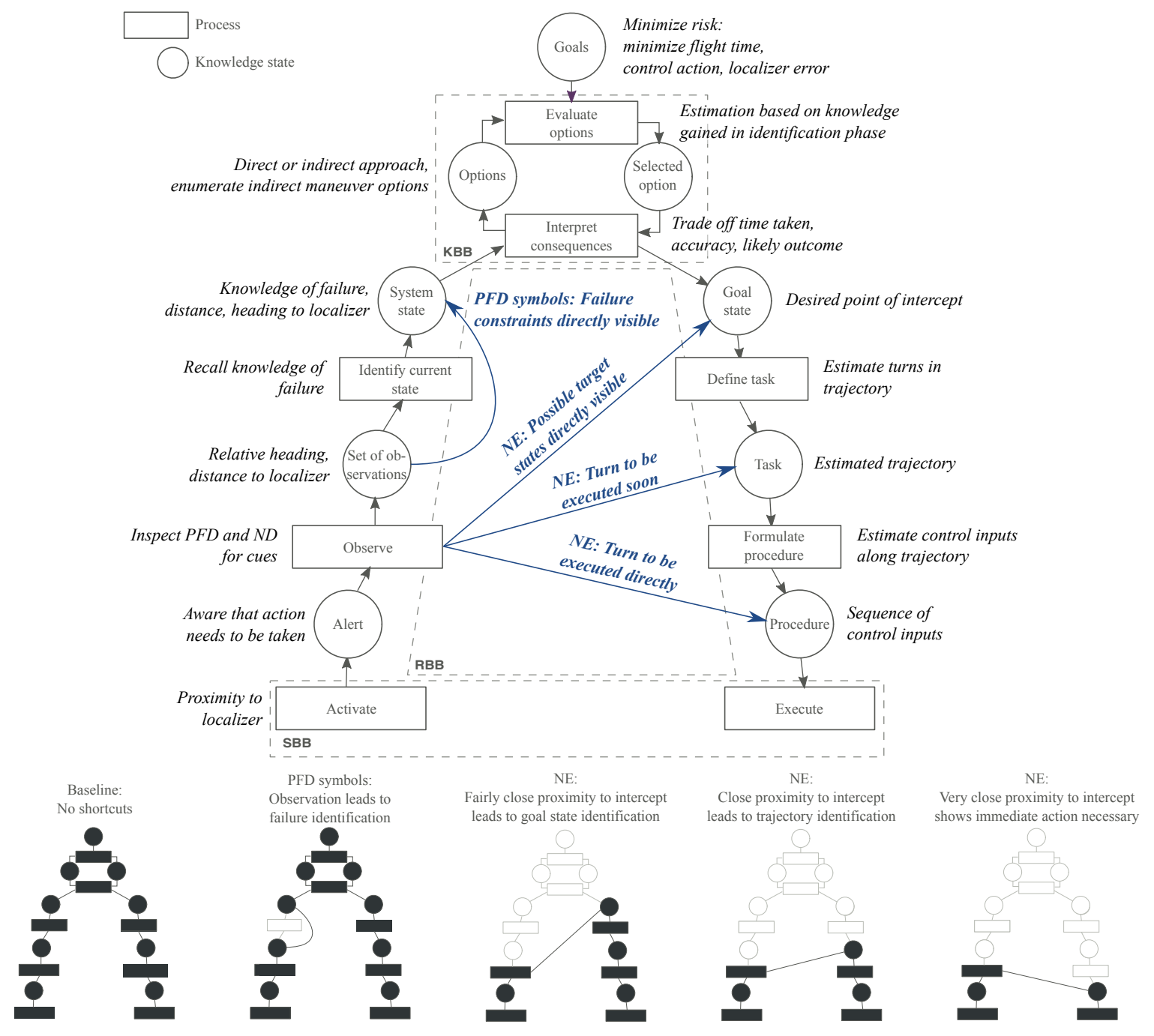

Fig. 8. Decision Ladder for a localizer intercept task. Adapted from [29].

TABLE II

CHRONOLOGICAL OVERVIEW OF PARTICIPANT ACTIVITIES

\begin{tabular}{|c|c|}
\hline $6 \mathrm{x}$ & $\begin{array}{l}\text { Receive briefing } \\
\text { Fly training runs } \\
\text { Identify failure } \\
\text { Select runway } \\
\text { Intercept localizer } \\
\text { Fill in workload assessment } \\
\text { Fill in questionnaire }\end{array}$ \\
\hline
\end{tabular}

1) Briefing: Initially, the pilots were briefed on the tasks they were about to perform and the interfaces that would be used during the experiment. The constraints of the experiment were also explained at this stage.

2) Training: After the briefing, a training phase commenced in which the participants were given the time to familiarize themselves with the different interface combinations and the dynamical model of the aircraft. At this point, no failure was present in the model and the model was linearized with the outboard ailerons as active control surface $\left(K_{p}=0.3984, \tau_{p}=1.130\right)$.

During the training phase, the participants were encouraged to track a reference trajectory consisting of multiple straight segments with increasingly sharper angles between them. This prompted the participants to exert a significant amount of control action such that they could clearly observe the behavior of the different interface symbols. Additional clarifications relating to the new symbology were still being provided at this stage.

During all phases of flight, participants were instructed not to exceed the visualized bank angle limits. In this event, a stall warning would be shown, the simulation would be halted, and the event would be treated as a loss-of-control event. The limits were not exceeded during any of the measurement runs.

3) Measurement runs: After the training phase, six trials were performed under different experimental conditions. The trials were split up in distinct parts, drawing inspiration from the GARTEUR FM-AG(16) benchmark [25]. These parts are:

- Failure identification 
- Runway selection

- Localizer intercept

- Workload assessment

First, the pilot was given the opportunity to identify the failure in flight, while not pursuing any other goals at the same time. The motivation for this was that in a real-life performance-altering event, the first thing a pilot would do would be to get an impression of the altered flight envelope.

Second, the simulation was halted and the aircraft was repositioned to a predetermined position in the vicinity of an airport with two runways and the pilot was asked to indicate which runway would be preferred for performing a landing, given the current aircraft state, the orientation of the runways, and the nature of the failure.

Third, the pilot was asked to perform a "raw data" (i.e. without flight director) localizer intercept. The pilot was instructed to perform the intercept in the best possible way while minimizing the flying time and the localizer error along a defined segment. It was indicated that as the cause of the failure was not known, the failure could potentially get worse. In practice, this meant the pilots were encouraged to only fly an indirect approach when they thought they would not be able to intercept the localizer sufficiently well by performing a direct approach. A direct approach as defined here consists of one turn towards the localizer and one turn to align with the localizer. The participants were instructed to complete the localizer intercept at or before the nearest waypoint shown on their ND if they could do so, and to keep minimizing the error until the second waypoint (representing the glide slope capture) was reached. The simulation was halted when this waypoint was passed.

As the fourth and last step of each measurement run, the participant was asked to complete a workload self-assessment by means of filling in a NASA TLX form [34].

\section{Apparatus}

The experiment was conducted in TU Delft's SIMONA research flight simulator. For this experiment, the 6 degree-offreedom motion base was not used. The participants controlled the simulation exclusively by means of a right-handed (first officer seat) control-loaded side stick. In front of the participant, two screens displayed the ND (left) and PFD (right) displays. An outside visual consisting of flat terrain was added for realism and providing optical flow cues, but no additional cues could be obtained from this visual.

\section{Independent Variables}

Two independent within-subject variables were defined for this repeated-measures experiment: the failure condition ('FAIL') which had two levels and the EFIS display configuration ('DISP'), which had three levels. Hence, in total there were six different experimental conditions in this experiment. A Latin square distribution was used to mitigate order effects.

1) Failure Condition: The two levels of the FAIL independent variable were named 'moderate' and 'severe'. These were different only in the amount of aileron offset required to maintain a constant bank angle, see Figure 9. Note that between the two levels, the aileron deflection was also mirrored. The safe maneuvering constraints as introduced in Figure 3 were calculated for both failure conditions; these are shown in Figure 10. In the direction of impaired roll authority - the most challenging aspect of the asymmetric envelope - the maximum rates were $1.41 \%$ s (left) and $0.70 \%$ s (right) for the moderate and severe failure condition, respectively.

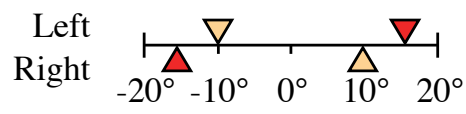

Fig. 9. Inboard aileron deflection for steady flight for moderate (inner triangles) and severe (outer triangles) case

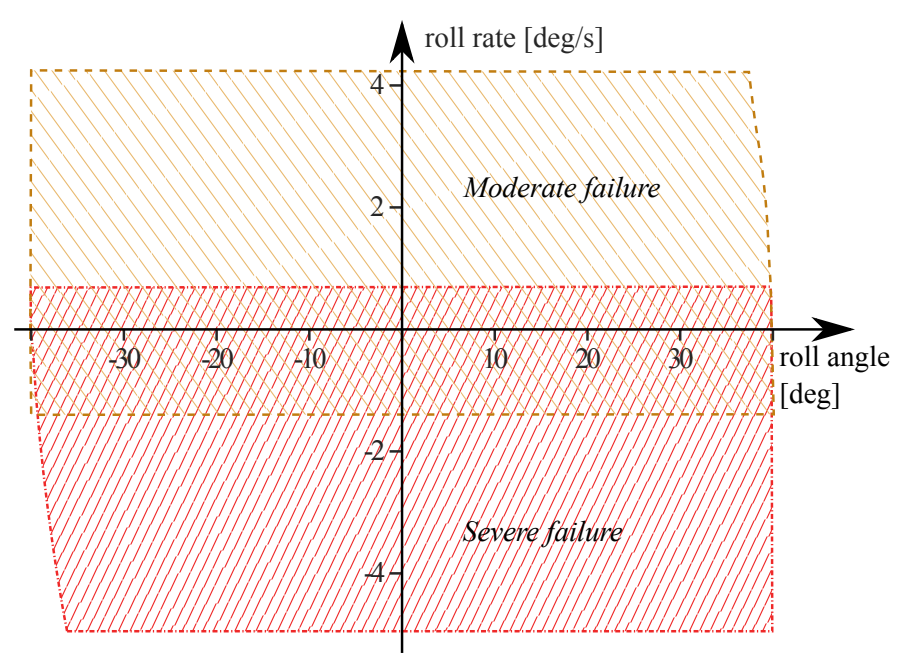

Fig. 10. Lateral maneuvering envelope for the two failure levels

2) Display Configuration: The three levels of the DISP independent variable were named 'Base', 'PFD+', and 'ND+'. The 'Base' or baseline configuration consisted of a standard PFD and ND with the addition of bank angle limits on the PFD in the form of "barber poles", representing the stall angle which was not to be exceeded. The PFD+ level added the PFD symbology that was introduced in Section IV, and the ND+ level included both the PFD and ND symbology. These different configurations are presented in tabular form in Table III. A screen capture of the actual PFD and ND with the additional symbology as it was used in the experiment is shown in Figure 11.

\section{E. Control Variables}

The parameters that were used to linearize the GARTEUR RECOVER model are listed in Table IV. Additionally, the resulting control parameters that were used in the experiment are shown in Table V. Wind was not present in the experiment. Furthermore, altitude was constrained in the experiment, i.e., the "combined system" that was introduced in Section III only allowed the pilot to perform coordinated turns at constant altitude. 

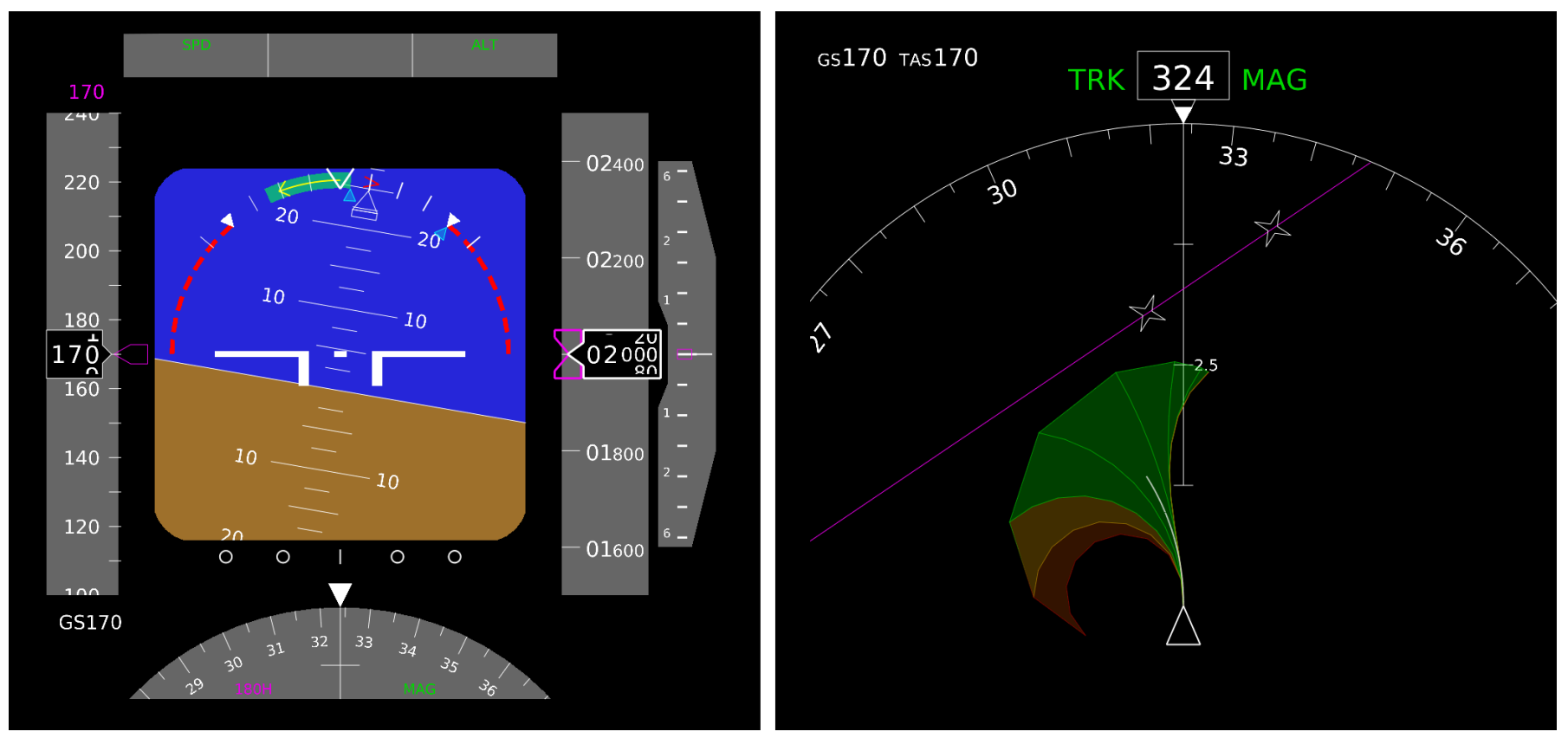

Fig. 11. Screen capture from PFD and ND used in the experiment, here showing the ND+ display condition

TABLE III

INTERFACE ELEMENTS AVAILABLE FOR THE DIFFERENT DISPLAY CONFIGURATIONS

\begin{tabular}{lccc}
\hline \hline & \multicolumn{3}{c}{ Display condition } \\
\hline & Base & PFD+ & ND+ \\
\hline Bank limits & $\checkmark$ & $\checkmark$ & $\checkmark$ \\
Roll rate limits & & $\checkmark$ & $\checkmark$ \\
Current roll rate & & $\checkmark$ & $\checkmark$ \\
Bank preview & $\checkmark$ & $\checkmark$ \\
Roll-out indicator & & $\checkmark$ & $\checkmark$ \\
Standard trend vector & $\checkmark$ & $\checkmark$ & $\checkmark$ \\
Navigation envelope & & & $\checkmark$ \\
\hline \hline
\end{tabular}

TABLE IV

LINEARIZATION PARAMETERS OF GARTEUR RECOVER BOEING 747 MODEL

\begin{tabular}{lc}
\hline \hline Parameter & Value \\
\hline Mass & $200,000 \mathrm{~kg}$ \\
Failure mode & 0 (none) \\
Gear & 0 (down) \\
Flaps & $20^{\circ}$ \\
Altitude & $2000 \mathrm{ft}$ \\
Speed & $170 \mathrm{kts}$ \\
Flight path angle & $0^{\circ}$ \\
\hline \hline
\end{tabular}

\section{F. Scenario}

The scenario is shown in Figure 12, where the aircraft symbol represents the initial position and heading, the diverging semi-shaded lines represent the two localizer centerlines leading to the two runways indicated at the top of the figure. Each localizer is marked with the two waypoints that were
TABLE V

CONFIGURATION OF EXPERIMENT MODEL CONTROL VARIABLES

\begin{tabular}{lc}
\hline \hline Parameter & Value \\
\hline$K_{p}$ & 0.141 \\
$\tau_{p}$ & $1.130 \mathrm{~s}$ \\
Altitude & $2000 \mathrm{ft}$ \\
Speed & $170 \mathrm{kts}$ \\
Flight path angle & $0^{\circ}$ \\
Bank angle limits & $\pm 40^{\circ}$ \\
Inboard aileron deflection limits & $\pm 20^{\circ}$ \\
\hline \hline
\end{tabular}

introduced in Section V-B3; these waypoints are also shown.

For the runway selection task, the participant was asked to indicate a preference for executing the localizer intercept on runway (1) or (2). It was indicated that the runway properties were similar in both dimensions and the available support, and that there was an absence of wind.

For the localizer intercept task, runway (1) was always "randomly selected" for performing the localizer intercept task in order to gather sufficient data points. In $50 \%$ of the trials, both the failure and the runway layout were mirrored along the North axis in order to prevent scenario and failure mode recognition. This is also why the runway layout was designed to be slightly asymmetric with respect to the initial position and heading.

\section{G. Dependent Measures}

The interfaces were evaluated using objective data originating from the logged data, as well as subjective data originating from NASA Task Load Index (TLX) workload assessments [35]. 


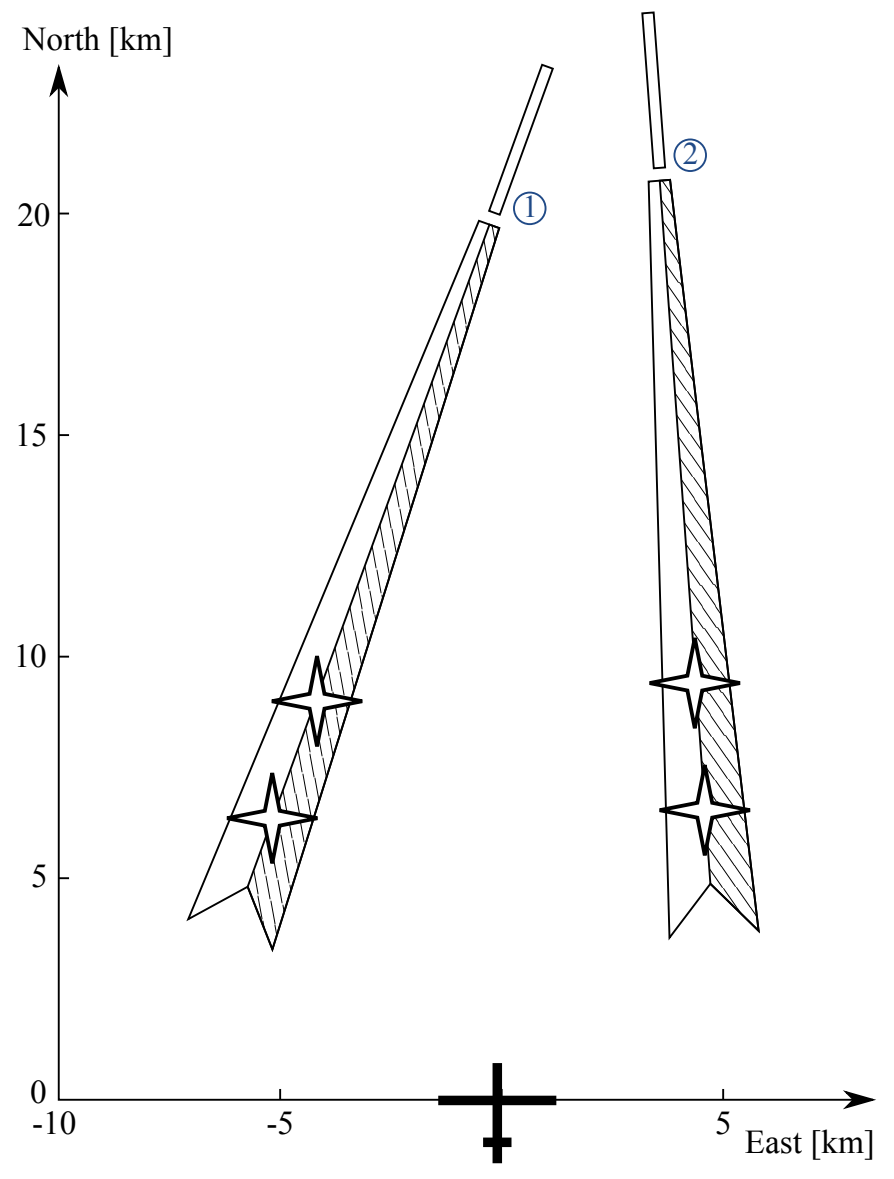

Fig. 12. Scenario used for runway selection task and localizer intercept. The intercept was always performed on runway (1).

1) Decision Quality: Two measures were used for assessing the quality of the decision: the preferred runway and the employed navigation strategy. Comparing the runway selection between the different conditions could indicate whether or not the display condition would have an impact on the preferred runway and the consistency in the preferred runway. The employed navigation strategy was characterized as a 'direct intercept' or an 'indirect intercept'. A direct intercept was defined as a trajectory consisting of two consecutive turns with a total flight time of less than 150 seconds. This strategy was deemed the preferred decision, as it was possible to perform a localizer intercept in this manner within the constraints of the experiment, and the pilots were instructed to employ this approach if they thought it would be feasible. The justification for this is that a direct localizer intercept would in this experiment result in a significantly lower total flight time, which would minimize the probability of sustained damage getting worse over time. For instance, the original investigation of Flight 1862 mentioned that the pilots may have believed that the starboard wing was on fire at the time. An indirect intercept was defined as any strategy resulting in a total flight time of more than 150 seconds.

2) Safety: Safety aspects were evaluated by the following metrics:

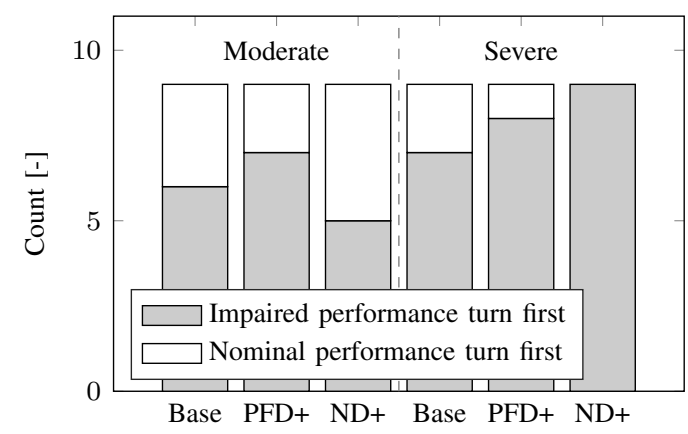

Fig. 13. Preferred runway selected for each condition

- Completion time. Time to completion was measured starting at the beginning of the intercept run until the aircraft crossed a line perpendicular to the localizer at the second waypoint. Lower completion times are more beneficial to safety as failures may propagate.

- Maximum bank angle during the intercept phase. Larger bank angles are closer to the boundaries of the safe flight envelope, leading to aerodynamic stall or insufficient thrust to maintain the current altitude.

- Maximum roll rate during the intercept phase. Higher roll rates cause larger structural loads and require more control action.

3) Performance: Performance was evaluated by the following metrics:

- Lateral localizer error at glide slope

- Heading error at glide slope

- Yaw rate at glide slope

For these metrics, lower values generally indicate that the pilot was more successful in tracking the localizer center line.

4) Workload: Following every localizer intercept phase, the participant was asked to assess his or her workload by completing a NASA TLX form [35]. The TLX calculates an overall workload metric based on ranking and grading the following categories: mental load, physical load, temporal demand, performance, effort, and frustration.

\section{H. Final Questionnaire}

At the end of the experiment, the participants were asked to complete a final questionnaire. The questions related to the pilot's self-assessed ability to infer meaning from the different interface elements. Furthermore, the participants were asked to comment on their concerns regarding clutter and training effects that may have been present during the experiment.

\section{REsults}

\section{A. Decision Quality}

First, the results from the runway selection task will be presented. Afterwards, the employed navigation strategies will be shown. 
1) Runway selection: Figure 13 shows whether the pilots preferred to perform a localizer intercept where the first turn would be in the direction of the slower or the faster roll rate. It can be seen that across all conditions, the pilots generally seemed to prefer to first turn with a slower roll rate. Every subject occasionally preferred the faster turn first without providing a very clear rationale on why this was done.

Cochran's $Q$ test was used to check for significant differences across the conditions with respect to runway preference. The results were not found to be significant $(Q(5,9)=$ $5.56, p=0.352$ ).

The pilots were asked to briefly describe how they would fly the approach, which confirmed these results: in most cases, the subject indicated to 'get the hard part out of the way first', i.e., initiate the first turn into the direction of the lower roll performance. In 51 out of 54 runs, the pilots estimated that they could fly a direct localizer intercept by performing two consecutive turns, while sometimes anticipating the potential need for changing this strategy to a more indirect approach, for instance by flying a so-called "teardrop" maneuver.

What was almost never part of the rationale, was the realization that 'getting the hard part out of the way first' would raise another issue: upon localizer capture, leveling out after the final turn would again require rolling the aircraft at an impaired rate, potentially causing the subject to miss the localizer intercept.

2) Employed navigation strategy: The flown trajectories can be seen in Figure 14. All trajectories started at $(0,0)$. The trials that were presented to the pilot in a mirror image have been transformed back in this figure in order to compare the results. Looking at the moderate failure case, we can see that with both the Baseline and PFD+ configuration, two pilots determined they could not fly the trajectory with a direct approach. With the ND+ configuration, every pilot decided the intercept could be flown directly. Furthermore, it seems that with the ND+ configuration the pilots opted for a larger intercept angle on average, enabling them to intercept in closer proximity to the first waypoint as requested.

Looking at the severe failure case, we can observe that both with the Baseline and PFD+ conditions, many pilots opted for an indirect approach, employing various strategies to intercept the localizer. With the ND+ configuration, pilot behavior was more consistent: only one pilot opted to fly away from the runway first. Cochran's $Q$ test was used for significance with respect to the localizer intercept strategy. The test pointed out that the display level had a significant effect on the chosen approach strategy $(Q(2,9)=8.4, p=0.015)$. However, a posthoc analysis with Bonferroni-corrected pairwise comparisons using McNemar's test did not yield a significant difference.

\section{B. Safety}

The employed strategy can also be observed in the time taken to intercept the glide slope: Figure 15 presents this data. The dotted line at 150 seconds represents the threshold above which an indirect approach was flown. Here, we can also see that as a result of the different indirect approaches in the severe failure case, both the median and spread of the data were higher when the ND+ display condition was not used. Friedman's ANOVA was used to test for significance for all safety and performance measures, as the necessary conditions for conducting parametric tests were not generally met [36].

DISP had no significant effect for the moderate failure case $\left(\chi^{2}(2)=2.667, p=0.264\right)$. A significant effect was found for the severe failure case $\left(\chi^{2}(2)=8.222, p=0.016\right)$. Here, a post-hoc analysis using Wilcoxon's signed-rank test showed that the ranks for the Baseline and PFD+ levels were significantly different $(Z=-2.547, p=0.011)$ at the Bonferroni-corrected significance level of $p=0.0167$.

Figure 16 shows the maximum roll rates that were registered during the intercept task. We can see that with the severe failure case, higher roll rates were commanded. Across the different display levels, the results seem more consistent. In fact, no significant effect was found for either the moderate failure level $\left(\chi^{2}(2)=2.889, p=0.236\right)$ or the severe failure level $\left(\chi^{2}(2)=2.889, p=0.236\right)^{1}$.

Finally, looking at Figure 17, we can see that the maximum bank angle that was obtained during the intercept runs was fairly consistent across the different display levels, especially for the moderate failure case $\left(\chi^{2}(2)=2.889, p=0.236\right)$. In the severe failure case, Figure 17 shows a lower median maximum bank angle for the ND+ level. While there was a significant effect on the maximum bank angle in the severe failure condition $\left(\chi^{2}(2)=6.889, p=0.032\right)$, one should be careful not to interpret this result as the NE causing more conservative steering behavior. Instead, in this scenario the NE made obvious that a left turn at a high bank angle would most likely result in failing to intercept the localizer, suggesting the pilots to execute the turn at a lower bank angle. Wilcoxon's signed-rank tests did not produce a significant effect for any of the pairwise comparisons.

\section{Performance}

The box plots in Figures 18 to 20 aim to explain how well the pilots managed to reach the glide slope in a stable and accurate manner. The three figures tell a similar story as, generally speaking, a large lateral error would typically drive a pilot to command a heading change towards the glide slope, which would also temporarily result in a non-zero yaw rate.

It can be observed that in general, the Baseline display level yielded the best performance in terms of the metrics presented here. This can be explained when we take another look at the trajectories in Figure 14: as the employed navigation strategy was indirect much more often in the Baseline display level, the pilots were able to intercept the localizer further away than when a direct approach was flown. The result of this is that they had more time on their hands to correct for any localizer error. This also explains why the yaw rate at the glide slope was often smaller in the severe failure case: again, in this case an indirect approach was selected more often.

For the lateral error, no significant effects were found for neither the moderate $\left(\chi^{2}(2)=1.556, p=0.459\right)$ or the severe

\footnotetext{
${ }^{1}$ Friedman's ANOVA indeed yielded the same results for both tests
} 

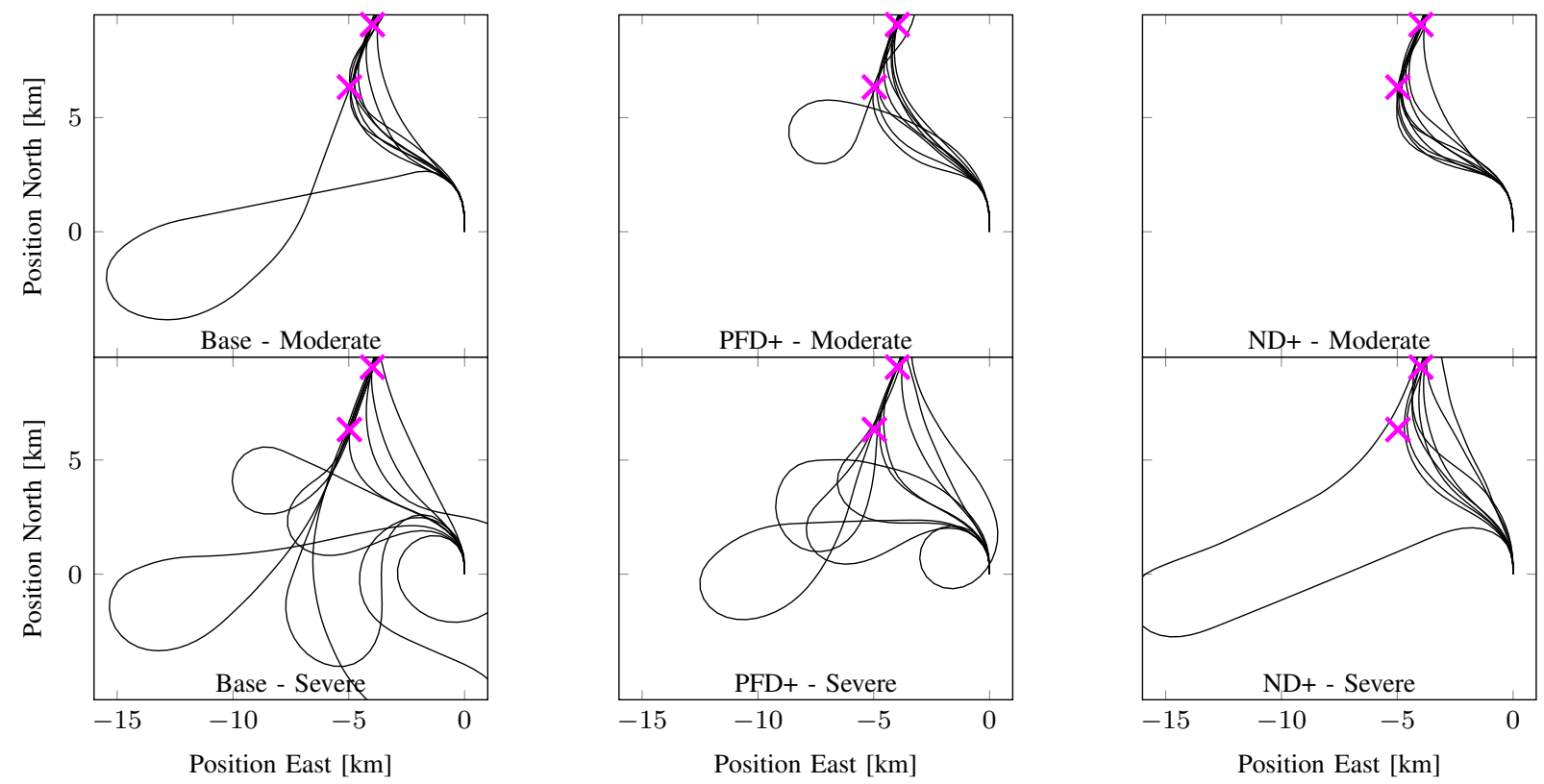

Fig. 14. Flown trajectories for the six experimental conditions

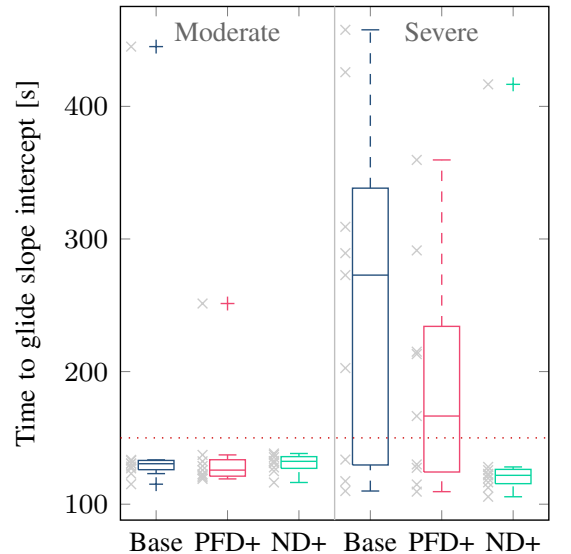

Fig. 15. Completion time for every subject

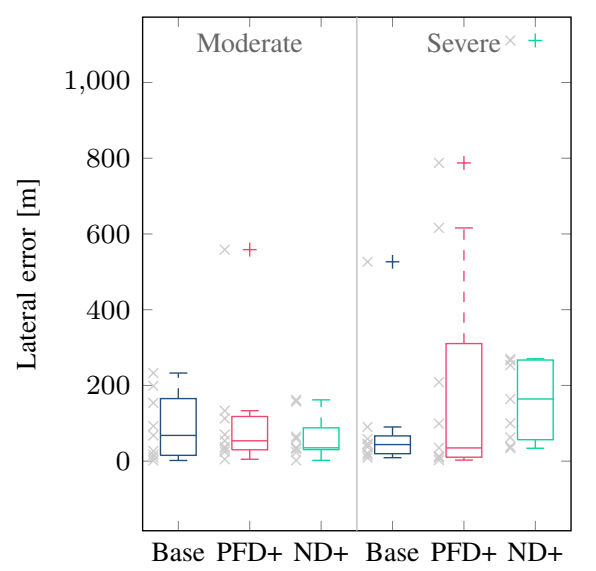

Fig. 18. Lateral error at glide slope

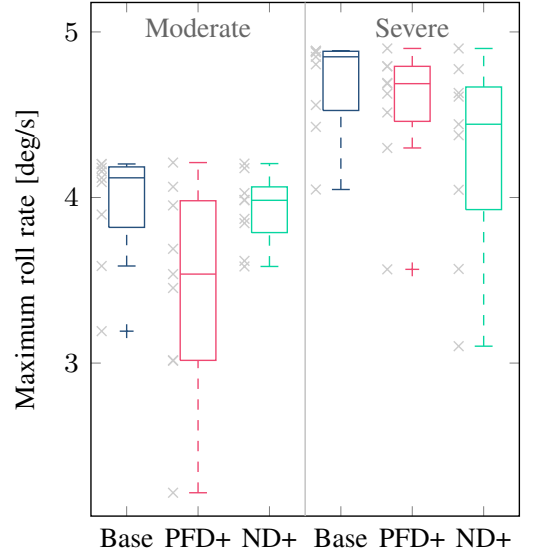

Fig. 16. Maximum roll rate

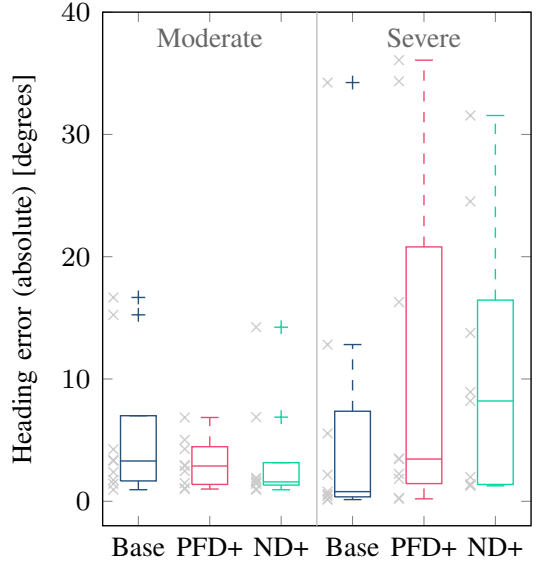

Fig. 19. Heading error at glide slope

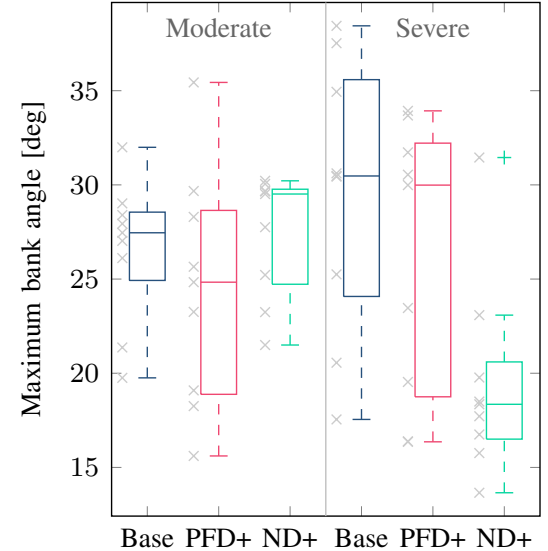

Fig. 17. Maximum bank angle

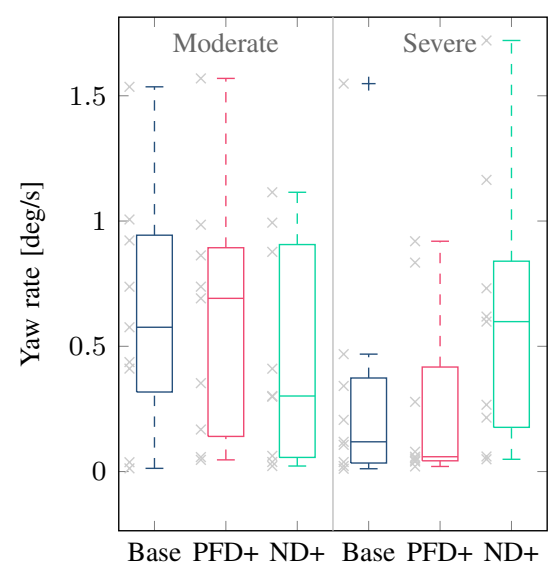

Fig. 20. Yaw rate at glide slope 
$\left(\chi^{2}(2)=0.222, p=0.895\right)$ failure level. The same is true for the heading error in both conditions $\left(\chi^{2}(2)=1.556, p=\right.$ 0.459 and $\left.\chi^{2}(2)=0.667, p=0.717\right)$, and for the yaw rate $\left(\chi^{2}(2)=2.000, p=0.368\right.$ and $\left.\chi^{2}(2)=0.889, p=0.641\right)$.

\section{Workload}

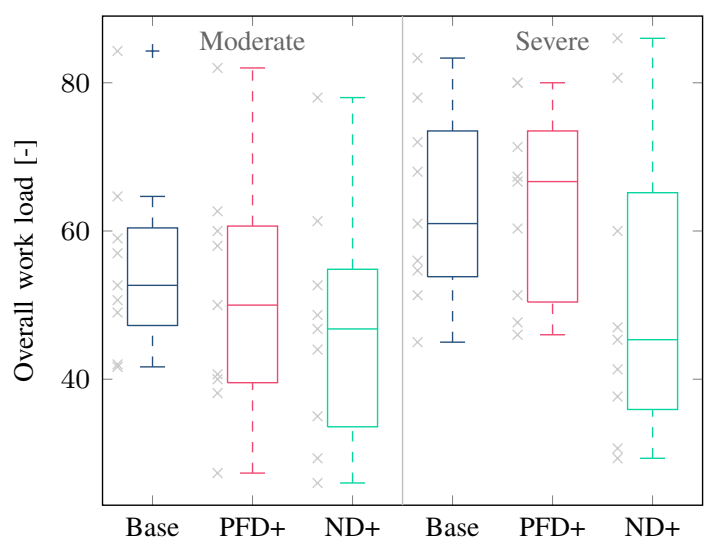

Fig. 21. NASA TLX overall workload as reported by the participants

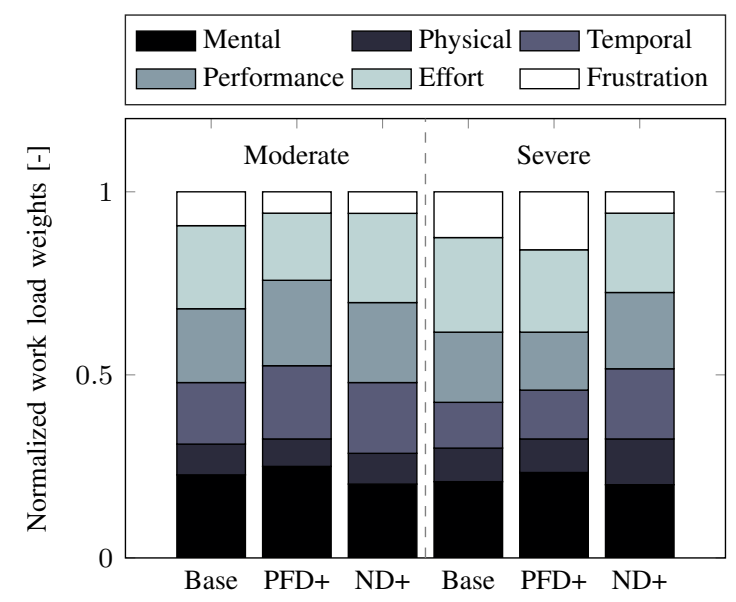

Fig. 22. TLX workload weights

Figure 21 shows the overall workload that was experienced by the participants according to the TLX results. Additionally, the importance weights of the different TLX categories are shown for the different conditions in Figure 22. The weights seem consistent across the experimental conditions, which implies that the nature of the task was not perceived fundamentally different as a result of the condition. We can observe that for both conditions with the ND+ level, both mental load and frustration were ranked slightly lower than in the other conditions, which may indicate that the pilots felt less discouraged and more secure when using the NE.

Looking again at Figure 21, the results show an increase in the median workload with the severe failure condition. This should be expected, as there was a lower margin of error for completing the task in an optimal way. The most obvious outcome is that the median reported workload was lower especially in the ND+ display configuration. This may imply that displaying integrated information indeed lowers the workload for the task.

Furthermore, we can observe that in the severe failure case, the PFD+ condition seemed to increase the reported workload. This could imply that the introduced symbology was creating more clutter than providing useful information. An analysis using Friedman's ANOVA with respect to the TLX overall workload did not yield a significant result $\left(\chi^{2}(5)=10.00, p=\right.$ 0.075).

\section{E. Questionnaire Results}

When asked whether the different interfaces improved or decreased their performance, the participants unanimously reported that mainly the modified ND improved their performance. This confirms that the pilots indeed favored a direct localizer intercept with a slightly larger error in terms of deviation and heading over taking additional time to intercept the localizer at a later point in time.

When asked which feature was the most useful for controlling the aircraft, the pilots found that for the PFD, the most useful feature was the green bar indicating the attainable roll rates. All pilots indicated that they relied on this feature to some extent. The other PFD features were all found to be of very limited use, perhaps introducing more clutter than providing useful information. The NE on the ND on the other hand, was deemed the most useful feature overall.

When asked whether the PFD or ND modifications aided the pilots in understanding the consequences of their control actions, a similar pattern was visible: all pilots found that the PFD modifications helped to some extent, but again the navigation envelope was the main driver of this aspect, with most pilots fully agreeing to the statement.

In terms of the runway selection task, the majority of the pilots thought the PFD modifications were not helpful in the runway selection task, while the majority thought the NE did help in this respect.

Most participants also felt that these or similar interface elements would help them in performing better during a performance-altering emergency, with one pilot making the remark that he thought this was only true with respect to the ND. The majority of the participants did not feel that the additions would introduce too much clutter on the displays.

\section{DISCUSSION}

\section{A. Methodology and Results}

Both the data gathered during the experiment as well as the results from the questionnaires seem to indicate that especially previewing position and heading based information on the ND allows pilots to develop a better understanding of reachable navigational states when they are confronted with off-nominal aircraft performance. Additionally, when the navigation options were harder to estimate (in the severe failure case) the workload assessments may indicate that the pilots felt more secure about the outcome of their actions. Lastly, the workload may decrease. This may be explained by recognizing that the 
Navigation Envelope allows pilots to make decisions using $\mathrm{RBB}$ instead of KBB when planning their actions, requiring a less significant cognitive effort.

While these results seem logical and promising, caution is necessary when interpreting the results from this experiment. One of the limitations of this experiment was the limited number of participants, which made it unfeasible to make bold statements about the population mean and variance of the measured signals. Especially combined with the fact that pilots were free to employ any navigation strategy they deemed appropriate, it is not possible to obtain sound statistical evidence from the obtained results; the results are mainly indicative in that they lend credibility to the idea that integrating aircraft performance parameters all the way up to position and heading based information can be a valuable source of information. Additionally, as the NE was not tested independently of the PFD modifications, one could argue that the interaction of the two displays could explain the improvement in pilot performance. However, based on the questionnaire results and pilots often citing they were barely paying attention to the PFD when the NE was visible, this seems unlikely.

One aspect that was evident both from observing pilot behavior as well as from the questionnaire results, was that not enough training time was provided to the subjects, i.e., the subjects felt that they became more proficient in using the new interfaces while they were performing the experiment. This implies that a larger amount of training time would likely have resulted in a better familiarity with the interface elements and perhaps better pilot performance through better apprehension of the presented symbology.

Additionally, the control task was limited in that the navigation task was purely two-dimensional in nature and the used aircraft model was rudimentary. This was required in order to constrain both the analysis and the experiment, but the question that remains is whether or not the presented interface elements can be modified to 1) provide sufficient and coherent information in the event of a more complex failure mode such as with a high-fidelity model of Flight 1862 and 2) encode three-dimensional position and flight path information. As flight envelopes - especially off-nominal ones - are highly nonlinear in nature, it is a legitimate concern whether or not constraint-based interface symbology can capture the set of all possible control actions in such a way that it is still possible to infer useful information from its representation.

Another concern is simulation realism: in this experiment, the control task was rather simple and well-defined, and the simulator's motion base was not in use. It is obvious that a real-life situation would be vastly more stressful. Hollnagel states that in unfamiliar situations with significant time pressure, operators tend to use a "scrambled" mode of control, where rational decision making does not necessarily occur [37]. If the experiment would have induced higher stress levels through improved realism, increased time pressure or other means, the results may have shown less effective use of the interface symbology.

Lastly, it should be pointed out that the applicability of the NE is limited in terms of the problems it may solve. It is a tool that is useful for short-term tactical planning and execution, i.e., ad hoc planning and navigation with a maximum of approximately two to three minutes or one to two turns. For planning more elaborate trajectories, it is likely that an interface based on this approach would require operator involvement (i.e. defining intermediate waypoints and assessing their feasibility), because given that a destination is within range, a large spectrum of maneuvers exist that will lead to the destination. The Emergency Landing Guidance System by Borst et al. demonstrated that an EID-based approach can work in an all engines inoperative condition [29], but when the work domain is not constrained by a negative energy rate, the set of navigation states is virtually unconstrained and only limited by the fuel available.

\section{B. Pushing the Envelope and Perceiving Uncertainty}

In Section VI it was mentioned that in the moderateND+ experimental condition, the pilots chose to intercept the localizer with a larger initial angle, resulting in both a better realization of the given instructions, but also in slightly more aggressive maneuvers. This behavior is consistent with observations in EID research, where operators decided to operate closer to the visualized limits of the system when these were present [29], [38]. It can be argued that operators presented with EID based interfaces have the tendency to "push the envelope", implying that ecological interfaces invite operators to utilize less safe behavior. However, this would be an oversimplification. Comans et al. have shown that operators are also compelled to honor intentional constraints (as opposed to physical constraints) when these are visualized, indicating that control behavior can be modified by introducing additional 'soft' constraints [38]. In case of the NE present in this study, one could imagine applying a different shading to indicate the bank angles that yield a certain minimum level of passenger comfort. Additionally, one could argue that knowingly operating close to a system's limit is perhaps safer than operating at a safer region in the state space without knowing where the system's limits are: with the NE present, the pilots were more certain that they could execute certain maneuvers, whereas without this information present, they were less certain about the outcomes of their control actions. A good interface should improve operator confidence without making the operator overconfident.

Operating close to system boundaries becomes a more pronounced issue when the system boundaries are less clear: the model that was used in this experiment was time-invariant, deterministic, and its parameters were assumed to be fully known by the system that was producing the interface. In reality, this is a luxury we do not have; safe maneuvering envelopes of aircraft have a high dimensionality and the space of all possible control inputs affects the energy balance of the aircraft, making it hard to visually or mentally chain together series of viable control actions. Furthermore, external factors such as wind direction and wind strength are hard to predict locally in an accurate manner, introducing additional uncer- 
tainties. Here, the interface designer will need to determine whether these uncertainties should be visualized in some form (again inviting operators to demonstrate less safe behavior), or to tighten the constraints (contracting the solution space).

\section{RECOMMENDATIONS}

Given that some data seems to be indicating that it may be worthwhile to present navigation-based constraints to pilots during performance-altering flight conditions, an effort should be made to bridge the gap between these findings and realworld situations. We suggest that follow-up research should be conducted, again using piloted simulations. In order to verify that the preliminary findings that were presented in this paper can be replicated, a suitable platform needs to be found that would enable researchers to perform additional experiments. One such platform could be the GARTEUR RECOVER benchmark, which is widely used in the field of FTFC algorithm development [10], [24]. Pilot performance assessment and interface evaluation may prove to be a natural extension to this program. However, the model's complexity may pose a significant drawback as analyzing and describing the model's flight envelope may prove to be unfeasible at this point in time, due to the high dimensionality and nonlinearity of the model. Alternatively, Lombaerts et al. have been using NASA's Generic Transport Model for maneuvering envelope estimation, which may prove to be a more manageable starting point for the purpose of interface design [18], [39].

Furthermore, it is well known that command-based displays have benefits in time-critical situations as they are less mentally demanding when compared to constraint-based interfaces. Research has been done in the field of (automated) emergency landing planning [7], [6], [40] and these efforts may prove to be a suitable alternative to a constraint-based approach. One could also imagine joining these two concepts, as a visualization of the work domain constraints may allow a pilot to verify action plans that were developed by the automation, thereby leveraging the pilot's expert knowledge to supervise the automation [41]. Such a combined approach could potentially improve the robustness of the combined humanmachine system by enabling the pilot to respond quickly to a performance-altering event while still being able to effectively monitor the automation and respond to automation failures should these occur.

Finally, it is recommended that uncertainty resulting from maneuvering envelope estimation data is to be incorporated in the interface elements. However, this would first require an adequate understanding of how uncertainties in estimated parameters manifest themselves in navigation states. The observed pilot behavior in this experiment seemed consistent with other experiments where EID interfaces were tested in that pilots may be driven to "push the envelope" when presented with an ecological interface. Research suggests that this behavior may be mitigated by visualizing intentional constraints rather than physical constraints [38]. This aspect is worth investigating in the domain of visualizing navigation states.
Relevant in this context is also the connection between (fault tolerant) control and dynamics: in real systems there is a tradeoff between controller robustness and stability. For instance, Airbus aircraft enable pilots to operate its aircraft in different modes depending on aircraft state and sensor data availability, giving pilots more authority when higher performance may be needed. In the context of performancealtering events, a relevant question is whether pilots should be given full control authority with lower stability margins or lower control authority with higher stability margins. This is relevant because the interface plays an important role in this control loop: if we can adequately convey information that aids pilots in deriving a most-likely-to-succeed set of actions, we may give them more authority because it opens up the solution space, potentially uncovering high quality solutions. For instance, in this experiment the maximum bank rates could have been limited such that aircraft performance would be more symmetric. While this could potentially improve the flown trajectories as pilots would be less "surprised" by the asymmetrical performance, it could also have a negative effect on pilot performance as not all available control authority would be available to the pilot. It is worthwhile to investigate this tradeoff while making interface design an integral part of this process.

\section{CONCLUSION}

This study looked into the effect of presenting flight envelope information both in terms of low-level data (e.g. attainable rates) and integrated information (i.e. navigation states) to pilots. A human-in-the-loop experiment was conducted to investigate the effects. While the results bear limited statistical significance, possibly due to the limited sample size of the experiment, the data seem to indicate that especially presenting pilots with information in terms of reachable positions and headings allows them to better estimate off-nominal aircraft navigation performance, thereby improving performance during a localizer intercept task. Furthermore, self-assessed workloads were observed to be slightly lower when this information was present. In future research, it is recommended that emphasis is placed on realism, modeling and representing parameter uncertainty, and mitigating limit-seeking behavior.

\section{REFERENCES}

[1] Y. Tang, E. M. Atkins, and R. M. Sanner, "Emergency flight planning for a generalized transport aircraft with left wing damage," in AIAA Guidance, Navigation, and Control Conference 2007, vol. 5, no. August, Hilton Head, 2007. [Online]. Available: http://www.scopus.com/inward/record.url?eid=2s2.0-37249022821\&partnerID=tZOtx3y1

[2] R. Watts, P. Tsiotras, and E. Johnson, "Pilot feedback for an automated planning aid system in the cockpit," in AIAA/IEEE Digital Avionics Systems Conference - Proceedings, 2009.

[3] R. Watts, H. Claus Christmann, E. Johnson, K. Feigh, and P. Tsiotras, "Development and evaluation of an automated path planning aid," Journal of Aircraft, vol. 49, no. 6, pp. 1774-1785, 2012.

[4] N. Meuleau, C. Plaunt, and D. E. Smith, "Emergency Landing Planning for Damaged Aircraft," in ICAPS-08 Scheduling and Planning Applications Workshop (SPARK), 2008. 
[5] N. Meuleau, C. Plaunt, D. E. Smith, and T. Smith, "An Emergency Landing Planner for Damaged Aircraft," in Proceedings of the Twenty First Innovative Applications of Artificial Intelligence Conference, vol. 21. AIAA Press, 2009, pp. 114-121.

[6] N. Meuleau, C. Neukom, C. Plaunt, D. E. Smith, and T. Smith, "The Emergency Landing Planner Experiment," in ICAPS-11, 2011, pp. 6067.

[7] T. L. Chen and A. R. Pritchett, "Development and Evaluation of a Cockpit Decision-Aid for Emergency Trajectory Generation," Journal of Aircraft, vol. 38, no. 5, pp. 935-943, 2001.

[8] L. Bainbridge, "Ironies of automation," Automatica, vol. 19, no. 6, pp. 775-779, 1983.

[9] L. Skitka, K. Mosier, and M. Burdick, "Does automation bias decisionmaking?" International Journal of Human-Computer Studies, pp. 9911006, 1999.

[10] M. H. Smaili, J. Breeman, T. J. J. Lombaerts, and D. A. Joosten, "A simulation benchmark for integrated fault tolerant flight control evaluation," in Collection of Technical Papers - AIAA Modeling and Simulation Technologies Conference, 2006, vol. 1, 2006, pp. 563-585.

[11] Netherlands Aviation Safety Board, "Aircraft Accident Report - El Al Flight 1862." 1992.

[12] M. Smaili and J. Mulder, "Flight data reconstruction and simulation of the 1992 Amsterdam Bijlmermeer airplane accident," Modeling and Simulation Technologies Conference, no. August, 2003. [Online]. Available: http://arc.aiaa.org/doi/abs/10.2514/6.2000-4586

[13] J. M. Urnes, "Dynamic Flight Envelope Assessment and Prediction," in AIAA Guidance, Navigation and Control Conference and Exhibit, no. 8 , Honolulu, Hawaii, 2008.

[14] J. Keller, R. McKillip, and S. Kim, "Aircraft flight envelope determination using upset detection and physical modeling methods," AIAA Guidance, Navigation, and Control Conference, pp. 1-21, 2009. [Online]. Available: http://arc.aiaa.org/doi/pdf/10.2514/6.2009-6259

[15] L. Tang, M. Roemer, J. Ge, A. Crassidis, J. Prasad, and C. Belcastro, "Methodologies for Adaptive Flight Envelope Estimation and Protection," in AIAA Guidance, Navigation, and Control Conference, no. August, 2009, pp. 1-14.

[16] R. Pandita, "Dynamic Flight Envelope Assessment with Flight Safety Applications," Ph.D. dissertation, University of Minnesota, 2011.

[17] C. C. De Visser, "Global Nonlinear Model Identification with Multivariate Splines," Ph.D. dissertation, Delft University of Technology, Zutphen, 2011

[18] T. Lombaerts, S. Schuet, K. Wheeler, D. Acosta, and J. Kaneshige, "Robust maneuvering envelope estimation based on reachability analysis in an optimal control formulation," 2013 2nd International Conference on Control and Fault-Tolerant Systems (Systol), pp. 318-323, 2013.

[19] D. Asadi, M. Sabzehparvar, and H. A. Talebi, "Damaged airplane flight envelope and stability evaluation," Aircraft Engineering and Aerospace Technology, vol. 85, no. 3, pp. 186-198, 2013. [Online]. Available: http://www.emeraldinsight.com/10.1108/00022661311313623

[20] P. Lu, L. Van Eykeren, E. J. van Kampen, C. de Visser, and Q. Chu, "Double-model adaptive fault detection and diagnosis applied to real flight data," Control Engineering Practice, vol. 36, pp. 39-57, 2015.

[21] P. Lu, E.-J. van Kampen, C. de Visser, and Q. Chu, "Nonlinear aircraft sensor fault reconstruction in the presence of disturbances validated by real flight data," Control Engineering Practice, vol. 49, pp. 112-128, 2016.

[22] Y. Zhang, C. C. de Visser, and Q. P. Chu, "Online Safe Flight Envelope Prediction for Damaged Aircraft: A Database-driven Approach," AIAA Modeling and Simulation Technologies Conference, no. January, pp. 1-14, 2016. [Online]. Available: http://arc.aiaa.org/doi/10.2514/6.20161189
[23] J. M. Maciejowski and C. N. Jones, "MPC Fault-tolerant Fligh Control Case Study: Flight 1862." [Online]. Available: http://wwwcontrol.eng.cam.ac.uk/Homepage/papers/cued control 7.pdf

[24] C. Edwards, T. Lombaerts, and H. Smaili, "Fault tolerant flight control," Lecture Notes in Control and Information Sciences, vol. 399, 2010.

[25] H. Smaili, J. Breeman, T. Lombaerts, and O. Stroosma, "A benchmark for fault tolerant flight control evaluation," in 4th European Conference for Aerospace Systems (EUCASS), 2011. [Online]. Available: http://www.eucass-proceedings.eu/10.1051/eucass/201306333

[26] G. J. J. Ruijgrok, Elements of Airplane Performance. Delft University Press, 2009.

[27] J. A. Mulder, Q. P. Chu, J. K. Sridhar, J. H. Breeman, and M. Laban, "Non-linear aircraft flight path reconstruction review and new advances," Progress in Aerospace Sciences, vol. 35, no. 7, pp. 673-726, 1999.

[28] J. Rasmussen and K. K. J. Vicente, "Coping with Human Errors through System-Design - Implications for Ecological Interface Design," International Journal of Man-Machine Studies, vol. 31, no. 5, pp. 517534, 1989.

[29] C. Borst, F. A. Sjer, M. Mulder, M. M. Van Paassen, and J. A. Mulder, "Ecological Approach to Support Pilot Terrain Awareness After Total Engine Failure," Journal of Aircraft, vol. 45, no. 1, pp. 159-171, 2008.

[30] C. Borst, M. Mulder, and M. M. Van Paassen, "Design and Simulator Evaluation of an Ecological Synthetic Vision Display," Journal of Guidance, Control, and Dynamics, vol. 33, no. 5, pp. 1577-1591, 2010.

[31] S. B. J. van Dam, M. Mulder, and M. M. van Paassen, "Ecological Interface Design of a Tactical Airborne Separation Assistance Tool," Ieee Transactions on Systems Man and Cybernetics Part a-Systems and Humans, vol. 38, no. 6, pp. 1221-1233, 2008.

[32] G. A. Mercado, M. Mulder, and M. M. van Paassen, "Analysis of Air Traffic Controller Workload Reduction Based on the Solution Space for the Merging Task," in AIAA Guidance, Navigation, and Control Conference, 2010

[33] J. Rasmussen, "Skills, rules, and knowledge; signals, signs, and symbols, and other distinctions in human performance models," IEEE Transactions on Systems, Man, and Cybernetics, vol. SMC-13, no. 3, pp. 257266, 1983.

[34] A. Cao, K. K. Chintamani, A. K. Pandya, and R. D. Ellis, "NASA TLX: software for assessing subjective mental workload." Behavior research methods, vol. 41, no. 1, pp. 113-117, 2009.

[35] S. G. Hart and L. E. Staveland, "Development of NASA-TLX (Task Load Index): Results of Empirical and Theoretical Research," Advances in Psychology, vol. 52, no. C, pp. 139-183, 1988.

[36] A. P. Field and G. Hole, How to design and report experiments, 2003.

[37] E. Hollnagel, "Context, cognition and control," Co-operative proces. management, cognition and information technology, pp. 27-52, 1998.

[38] J. Comans, C. Borst, M. M. van Paassen, and M. Mulder, "Risk Perception in Ecological Information Systems," Proceedings of 17th International Symposium of Aviation Psychology, no. July 2015, pp. 436-441, 2013.

[39] T. Lombaerts, S. Schuet, D. Acosta, J. Kaneshige, K. Shish, and L. Martin, "Piloted simulator evaluation of maneuvering envelope information for flight crew awareness," in AIAA Guidance, Navigation, and Control Conference, 2015

[40] E. M. Atkins, I. A. Portillo, and M. J. Strube, "Emergency Flight Planning Applied to Total Loss of Thrust," Journal of Aircraft, vol. 43 no. 4, pp. 1205-1216, 2006

[41] C. Borst, J. M. Flach, and J. Ellerbroek, "Beyond Ecological Interface Design: Lessons From Concerns and Misconceptions," IEEE Transactions on Human-Machine Systems, vol. 45, no. 2, pp. 164-175, 2015. 


\section{Part II}

\section{Thesis Book of Appendices}





\section{Appendix A}

\section{Literature Study}

This literature study will commence with an introduction of El Al flight 1862, the fatal flight that will be used as a case study throughout this research project. Then, a brief introduction will be given on fault tolerant flight control. Afterwards, the concept of a flight envelope will be made along with analysis methods.

As this research project employs the EID framework, the literature study will continue with a description of the EID framework. Lastly, it was found that this research project would benefit from a survey that should indicate which efforts are underway in order to see what body of knowledge already exists on the subject of interface design for emergency landing planning. This short survey concludes the literature study of this preliminary thesis.

\section{A-1 El Al flight 1862, a Case Study}

Because of the relevance to the research that is being conducted and detailed knowledge of the aircraft's dynamics both pre- and post-failure, El Al flight 1862 is being used as a case study. This section will describe the events that occurred, followed by a description of the failure that occurred and possible argumentation for the flight crew's decision making strategy.

\section{A-1-1 Sequence of Events}

Source: (Netherlands Aviation Safety Board, 1992). On October 4th 1992, cargo flight El Al 1862 departed from Amsterdam Schiphol airport (AMS). Shortly after takeoff, around 17:27:30, while the aircraft was climbing through $6,500 \mathrm{ft}$, the flight crew noticed a sudden tendency of the aircraft to bank and turn right. Within the first minute, a mayday call was issued by the flight crew, fuel was being dumped, and a fire on engine 3 and loss of thrust on engines 3 and 4 were reported. The flight crew requested a direct return to AMS.

For about eight minutes, the crew struggled to keep the Boeing 747-200 under control as they flew an approach to runway 27 (see Figure A-1). This runway was requested by the crew; 
Literature Study

runway 06 was the runway in use because of the wind condition at the time. The crew tried to follow the vectors provided by air traffic control, but did not fully succeed: during the approach, flap problems were reported and a little while later, 'control problems' were also mentioned.

On 17:35:42, El Al 1862 crashed into an eleven-story apartment building in the Bijlmermeer area, taking the lives of the captain, first officer, flight engineer, one non-revenue passenger, and 43 people on the ground.

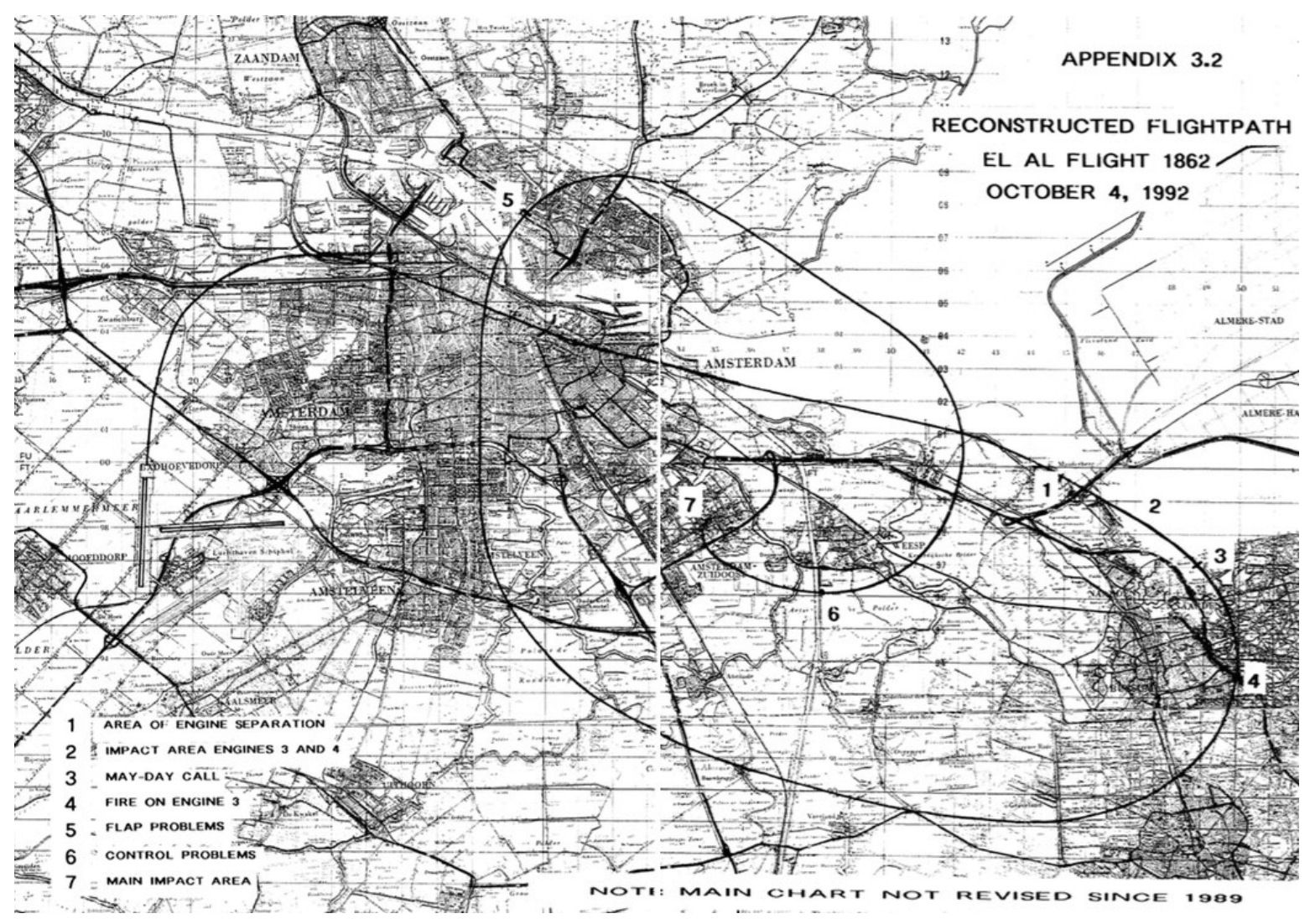

Figure A-1: The trajectory of flight 1862 (Netherlands Aviation Safety Board, 1992)

\section{A-1-2 Failure Description}

Subsequent analysis showed that during ascent, engine number 3 (inboard right) had detached from the right wing. In the process, the engine hit the leading edge of the right wing as well as engine number 4 (outboard right), which then also detached. The new force and moment equilibrium for steady straight flight can be seen in Figure A-2. The impact also affected hydraulics systems 3 and 4 in the wing, rendering multiple control surfaces unusable (see Figure A-3).

The original investigation mentioned that at higher angles of attack, the difference in lift between the left and right wing would increase as a result of the right wing leading edge damage. Therefore, increasing the angle of attack would generate a roll moment (right wing down) (Netherlands Aviation Safety Board, 1992). 


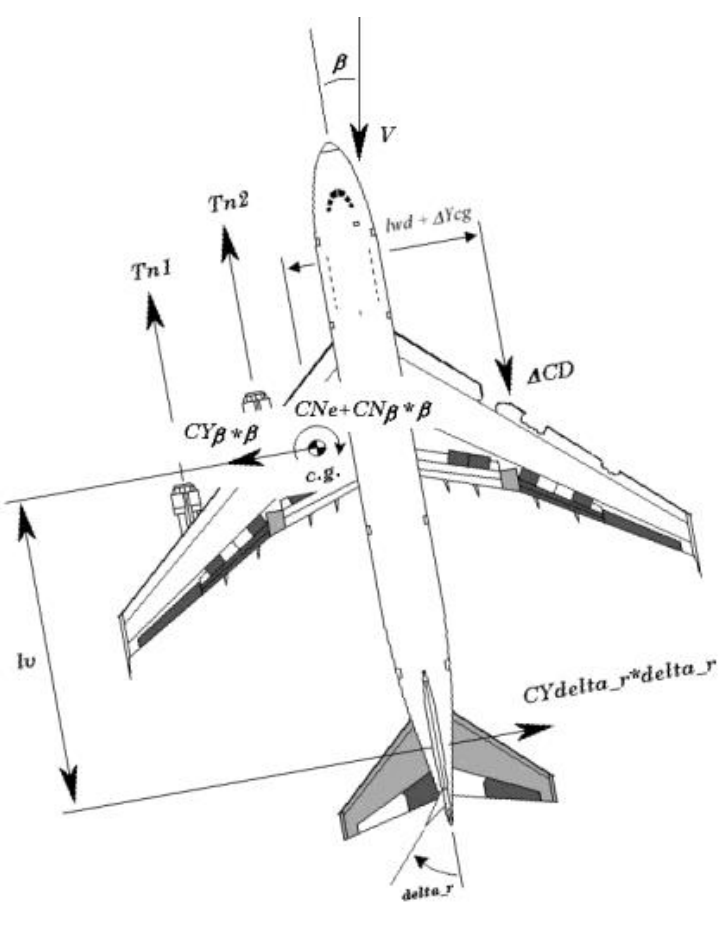

(a) with sideslip, no bank

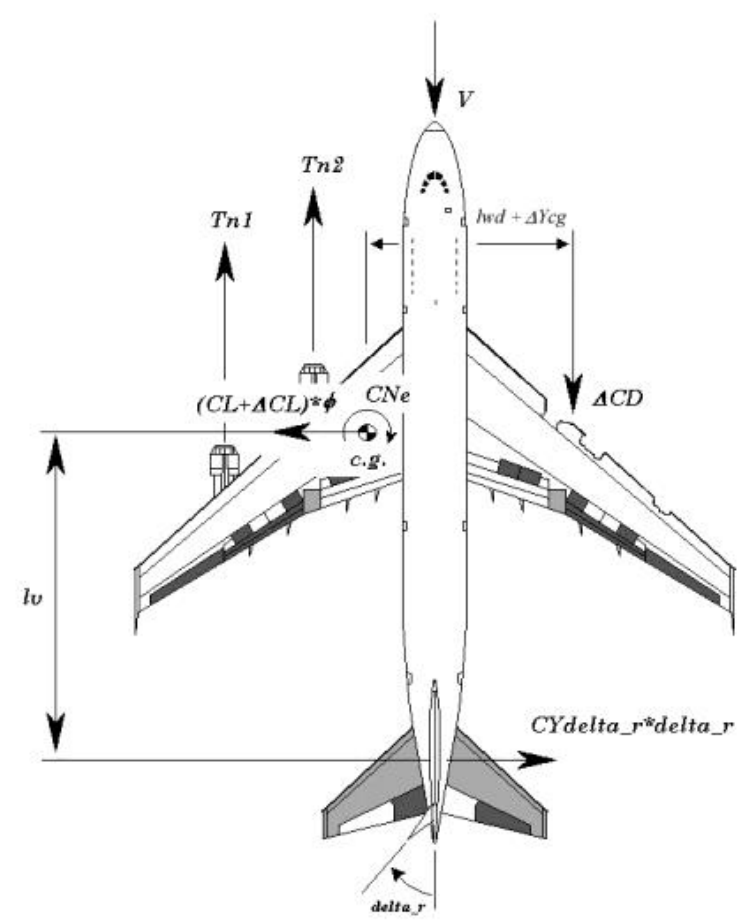

(b) with bank, no sideslip

Figure A-2: Force and moment equilibrium of Flight 1862 in straight flight with wing damage and engine separation (M. Smaili \& Mulder, 2003)

At the final stage of the flight, the angle of attack was increased, probably in order to reduce the descent rate. This led to a further increase in drag on top of the higher than nominal drag caused by the sideslip angle (see Figure A-2) and an additional decrease in speed. This probably caused the pilots to increase the engine thrust. This series of events led to a high roll moment (right wing down) due to asymmetric lift, asymmetric thrust, and reduced right hand inboard aileron efficiency. At this point, control of the aircraft was lost (Netherlands Aviation Safety Board, 1992).

\section{A-1-3 Decision Making}

Also relevant to this research are the considerations that served as inputs to the decision making process, as they were hypothesized by the NASB. One of the decisions in the emergency landing planning that stood out was that the crew decided to land on runway 27 with a tail/crosswind approach instead of the runway in use, runway 06 , which offered a headwind approach. While there is no evidence at all that these factors were indeed contributing to the decision making process at the time, it is not hard to imagine they may have been or that they may have been factors in comparable emergency situations. Together, these aspects give a good indication of the complexity of the decision making process.

The following factors may have caused the flight crew to decide to land on runway 27 (Netherlands Aviation Safety Board, 1992): 


\begin{tabular}{|l|}
\hline \multicolumn{1}{|c|}{ El Al Flight 1862 Failure Mode Configuration } \\
\hline Aircraft Systems \\
\hline Hydraulic systems 3 and 4 off \\
\hline Engine 1 and 2 thrust asymmetry \\
\hline Lower rudder lag \\
\hline \\
\hline Mass Properties \\
\hline Engine no. 3 and 4 weight loss, $4,014 \mathrm{~kg}$ each \\
\hline Pylon no. 3 and 4 weight loss, $\pm 1,000 \mathrm{~kg}$ each \\
\hline Lateral center of gravity displacement \\
\hline Total weight loss: $10,0028 \mathrm{~kg}$ \\
\hline \multicolumn{2}{|c|}{ Control surface lost } \\
\hline Aerodynamics \\
\hline Lift loss due to wing damage $\left(\Delta \mathrm{L}_{\text {wing }}\right)$ \\
\hline Rolling moment due to wing damage $\left(\Delta \mathrm{L}_{\text {wing }}\right)$ \\
\hline Drag due to wing damage $\left(\Delta \mathrm{D}_{\text {wing }}\right)$ \\
\hline Yawing moment due to wing damage $\left(\Delta \mathrm{N}_{\text {wing }}\right)$ \\
\hline Pitching moment due to wing damage $\left(\Delta \mathrm{M}_{\text {wing }}\right)$ \\
\hline $\begin{array}{l}\text { Right inboard aileron and spoiler } 10 \text { and } 11 \\
\text { aerodynamic efficiency loss }\end{array}$ \\
\hline
\end{tabular}

Figure A-3: Damage configuration of flight 1862 (M. H. Smaili et al., 2006)

- the possiblity of being hit by a missile, which would increase the likelihood of increasing damage over time

- the diagnosis of engine fire, which would also increase urgency as the fire could potentially burn the wing, increasing damage over time

- the assumption that the now failed aircraft was too heavy to sustain straight and level flight as the aircraft had just taken off

- runway 27 was the longer runway, which would increase the (perceived) probability of not overshooting the runway upon landing ${ }^{1}$.

Most of the factors that were mentioned would require a landing that would be as fast as possible. The NASB therefore concluded that the decision to land on runway 27 was understandable given the circumstances.

The NASB also made several recommendations, one of which had to do with the aircraft being directed over the densely populated city of Amsterdam. The board recommended that the safety of people on ground should also be taken into consideration when planning. This recommendation was mainly intended for ATC, but it is easy to see how the pilots could potentially have factored this information into their decision making process.

\footnotetext{
${ }^{1}$ It is not necessarily a given that this would be a good decision, but research has demonstrated that pilots have are biased to prefer longer runways over stronger headwinds in emergency conditions (Meuleau, Neukom, Plaunt, Smith, \& Smith, 2011), which may not be a good call but will nevertheless enter the decision making process.
} 
In an independent joint effort between TU Delft the Dutch Aerospace Laboratory (NLR), a simulation model was developed based on the original Digital Flight Data Recorder (DFDR) data (M. Smaili \& Mulder, 2003). Based on this simulation model, it has been stated that from a technical point of view, it would have been possible to land the aircraft safely (Edwards, Lombaerts, \& Smaili, 2010; M. Smaili \& Mulder, 2003). Maciejowksi and Jones demonstrated that given perfect knowledge of the failure by means of an FDI system and a model predictive control (MPC) system with the authority to actuate the individual control surfaces and remaining engines of the aircraft, it was possible to execute a safe descent to ground level (Maciejowski \& Jones, n.d.). However, a precision approach to one of Schiphol Airport's runways was not part of the simulation.

In hindsight, the question that remains is whether the pilots, even when equipped with a fault tolerant controller, would have been able to derive a navigation strategy that would result in a safe landing. Part of this problem lies in effectively communicating the reduced flight envelope limits and its relation to the environment to the flight crew and helping them understand the challenges they are facing.

\section{A-2 Fault Tolerant Flight Control}

The main goal of fault tolerant flight control is to improve survivability and recoverability after system faults and/or aircraft damage have occurred. In order to achieve this, FTFC systems aim to utilize the authority of the remaining control effectors (i.e. control surfaces and engines) to control the aircraft in all degrees of freedom (Edwards et al., 2010).

With the exception of passive FTFC systems, fault knowledge is required. In the development of FTFC algorithms, it is typically assumed that this fault information can be provided by a Fault Detection and Isolation (FDI) or Fault Detection and Diagnosis (FDD) module (see Figure A-4). This information is then passed to the FTC module, that optimizes the model parameters and perhaps reconfigures the controller based on this data. One important aspect to keep in mind is that no matter how 'smart' a controller is, there is no magic involved: when an aircraft suffers from a performance-altering fault or failure, performance will, by definition, be altered. A good controller may improve system stability and make it easier for the aircraft to be controlled, but the flight envelope will nevertheless be reduced.

Flight 1862 has served as the basis for the GARTEUR RECOVER benchmark (Edwards et al., 2010). This benchmark features a high fidelity Boeing 747 simulation model, which includes a model of the Flight 1862 failure and additional failure modes. The benchmark itself specifies a scenario based on the event and it serves to design, test, and compare different FDD modules, on-line model identification methods, and fault tolerant control systems. The approach profile of the GARTEUR FM-AG(16) benchmark and the corresponding phases of flight are shown in Figure A-5.

Many FDD and FTFC systems that are being developed and tested mainly try to serve the pilot by presenting him or her with an interface to the control effectors that behaves as though the mapping from control input to effector action is linear, thereby simplifying the control task (Maciejowski \& Jones, n.d.). One aspect that should be stressed is that as long as pilots are part of the equation (which they are as long as emergency flight planning and execution are not fully autonomous endeavours), their skills related to strategic thinking, planning, and 


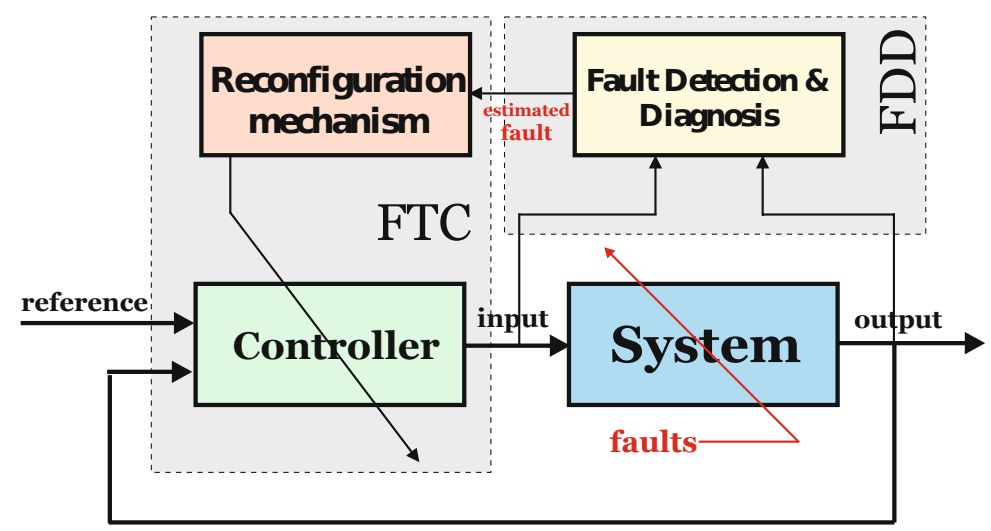

Figure A-4: Components required for an active fault tolerant control system (Edwards et al., 2010)

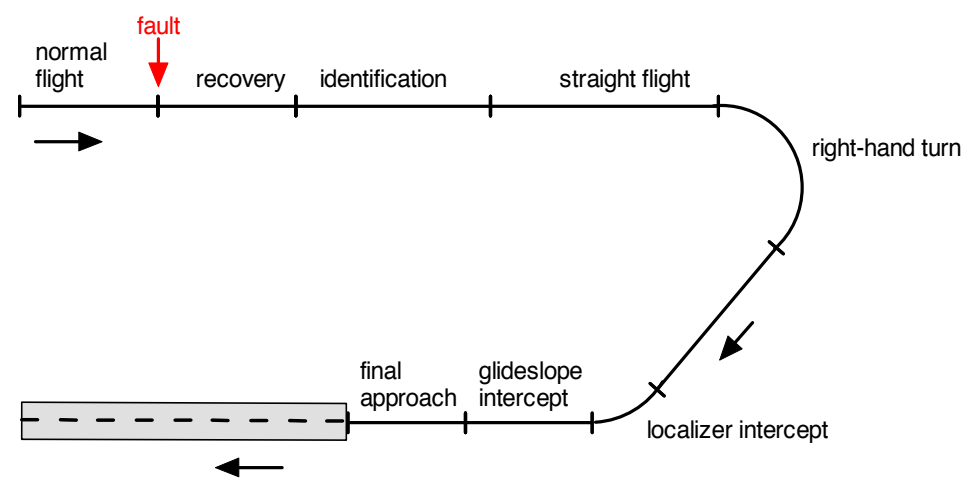

Figure A-5: GARTEUR FM-AG(16) benchmark approach profile (H. Smaili et al., 2011)

reasoning should be put to good use. This can not be done by algorithm development only: on the contrary, adding automation typically introduces more complexity. An effort should be made to keep pilots in the loop and to provide them with information that is relevant to the problem being solved. An analysis of the work domain based on the EID framework should indicated what this information entails.

Fault tolerant controllers may improve aircraft controllability, envelope protection systems may keep pilots and/or lower level control systems from flying outside the flight envelope, but these aspects by themselves do not guarantee that an aircraft will be navigated to a safe landing area and a safe landing will be executed. More specifically, in a detailed analysis of El Al flight 1862 conducted by TU Delft and the Netherlands National Aerospace Laboratory NLR, it was recommended to improve pilot awareness of performance and controllability limits as this may increase aircraft survivability (M. Smaili \& Mulder, 2003).

\section{A-3 Flight envelopes}

A well-known narrow definition of the flight envelope is that is consists of the combination of airspeeds and altitudes within which an aircraft can operate (Ruijgrok, 2009). In this 
definition, 'to operate' is typically defined as being able to maintain level flight. The equally well-known representation of this concept is the so-called 'doghouse plot' or $V-h$ diagram in which this relation is visualized.

An alternative means of defining the flight envelope's boundary is with the $V-N$ diagram, which shows the relation between the equivalent airspeed and the load factor that can be sustained by the aircraft as a function of the given airspeed. An example of such a diagram is presented in Figure A-6. The figure demonstrates that at low velocities, the maximum load factor is defined by the angle of attack at which stall occurs, whereas at higher velocities (beyond $V^{*}$ in the figure), structural limits dictate the load factor that should not be exceeded. The contained area is sometimes referred to as the maneuvering envelope of the aircraft.

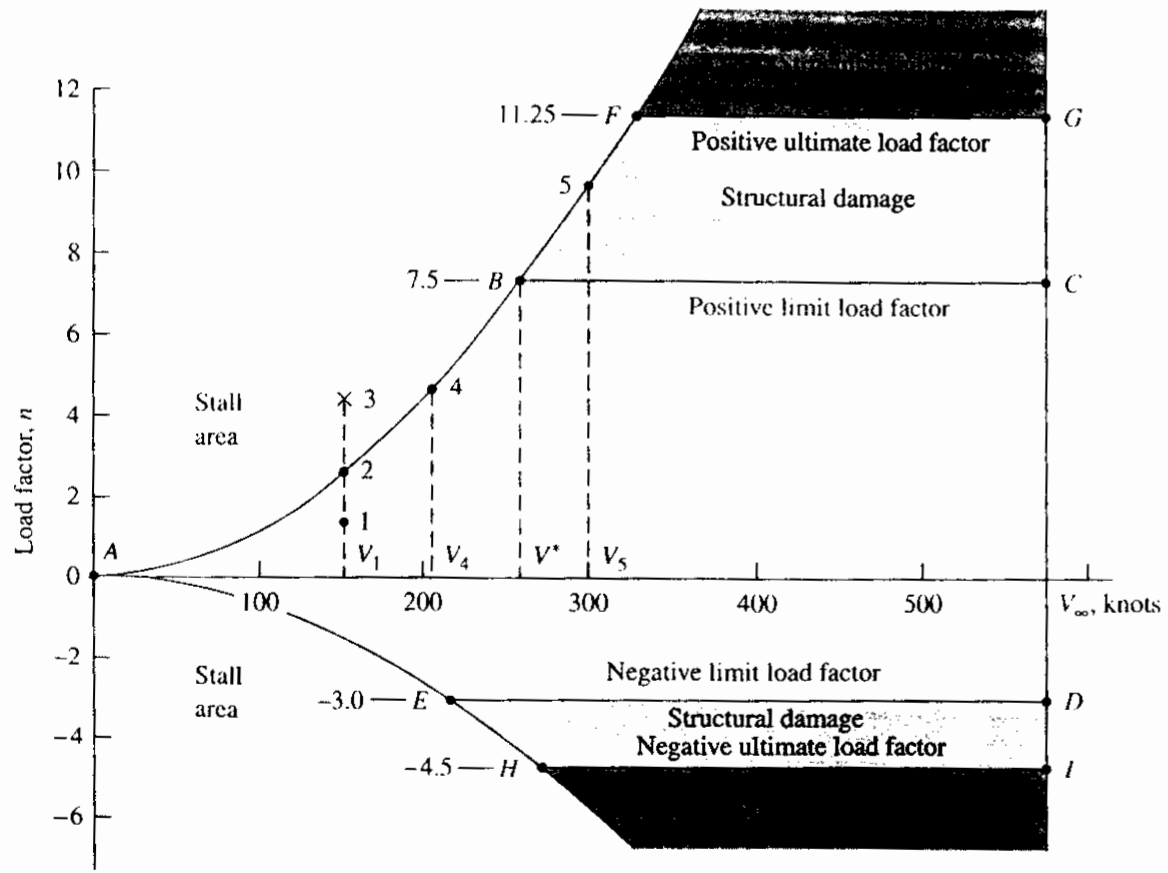

Figure A-6: V-N diagram for a typical jet trainer aircraft (Anderson, 1999)

We now have seen two concepts that aim to capture the flight envelope of an aircraft. The $V-h$ diagram defines the regime in which steady, straight, and level flight is possible. On the other hand, the $V-n$ diagram defines the relation between airspeed and load factor and whether or not an aircraft can sustain flight at this operating point without stalling or failing. Unfortunately, these analysis methods do not explain the dynamic behavior of aircraft as it transitions from one state to the next as a result of manipulating the control inputs. As a result, these methods fall short when it comes to describing the short-term navigation capabilities of aircraft in general. Neither do they provide insight into how the maneuvering capabilities are affected by changes in control surface effectiveness or dynamics. A different analysis method is thus needed to describe these capabilities.

One approach to describe the flight envelope of an aircraft is by identifying all possible trim states. Trim states describe states of non-accelerating flight. More precisely, a state is a trim 
state when all linear and angular accelerations are zero, i.e.:

$$
\begin{aligned}
& \dot{u}=\dot{v}=\dot{w}=0 \\
& \dot{p}=\dot{q}=\dot{r}=0
\end{aligned}
$$

Trim states can be calculated for different aircraft configurations (e.g., flap setting, gear setting) and flight conditions (e.g., altitude, mass, bank angle). Solving the aircraft equations of motion along these different configurations and flight conditions yields a trim database, which can then be used to describe the flight envelope - or, more precisely, the trim envelope - of the aircraft.

In trim calculations, dynamic behavior is not considered. In order to broaden the extent of a flight envelope, Van Oort, Chu, and Mulder have defined a useful categorization of flight regimes (Oort, Chu, \& Mulder, 2011). They defined a trim envelope and a safe flight envelope $^{2}$. The trim envelope is a subset of the safe flight envelope. It contains all the states in which the (state) derivatives of the linear and angular velocities can be controlled to zero. That is, there exists a set of control inputs that, when kept constant, causes the linear and angular velocities to remain constant. The safe flight envelope consists of the intersection of a backwards reachable set or survivable flight envelope and a forwards reachable set. The backwards reachable set is the set of states from which the initial set can be reached within a predefined time interval, and the forwards reachable set is the set of states the aircraft can be directed to with a state inside the initial set as initial state, again within a predefined time interval. This is shown graphically in Figure A-7. It should be noted that the trim envelope is a logical candidate for the initial set.

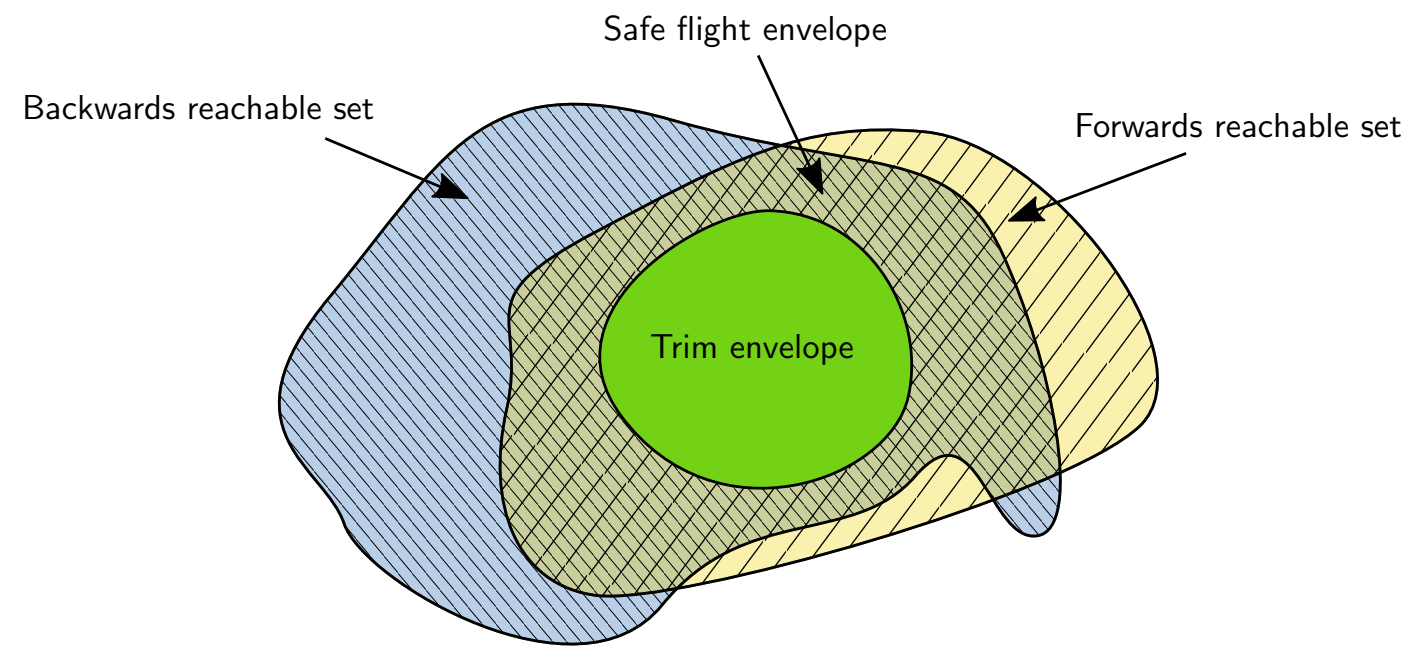

Figure A-7: Safe flight envelope representation, based on (Oort et al., 2011; Lombaerts et al., 2013)

\footnotetext{
${ }^{2}$ Actually, they defined a safe maneuver(ing) envelope. Subsequent research by Lombaerts et al. adopted the term safe flight envelope (Lombaerts, Schuet, Wheeler, Acosta, \& Kaneshige, 2013). Van Oort et al. defined the safe maneuver envelope as the intersection of the dynamic flight envelope, the structural and comfort envelope (including load limits), and the environmental envelope (including terrain and no-fly zones). In their paper the latter two envelopes were not included in the analysis. In this document, the term 'safe flight envelope' will be used to indicate the dynamic flight envelope in order to adopt the terminology used by Lombaert et alii.
} 
The key aspect here is that the safe flight envelope contains states that are not trim states, i.e., it is possible to safely maneuver the aircraft into these states, but they can not be maintained for extended amounts of time. For instance, a small but finite pitch rate can be maintained for a couple of seconds - in fact, it is required to realize a change in pitch attitude - but a state with a finite pitch rate is not a trim state as this would ultimately result in a loss of lift.

This illustrates that aircraft locomotion can not be fully explained by trim conditions only. Unfortunately, the process of analyzing safe non-trim states is harder and computationally intensive than calculating trim states. It remains an open question whether or not a representation of the full set of trim conditions can serve as a proxy for the true maneuvering capabilities and, by extension, the navigation strategies a pilot can develop based on this information in case of an emergency condition. Previous research on emergency flight planning does suggest that limiting the emergency flight planning problem to sequences of trim states is not overly restrictive (Tang et al., 2009; Strube, 2005; Atkins, Portillo, \& Strube, 2006).

\section{A-4 Ecological Interface Design}

Ecological Interface Design is the name of a framework that was developed by Jens Rasmussen and Kim Vicente (Rasmussen \& Vicente, 1989). Its goals are to support different levels of cognitive control (skill-based, rule-based, and knowledge-based behavior) and to not force cognitive processing to a higher level than necessary. EID was specifically developed to support complex work domains.

While EID originally emerged in the field of nuclear process control, the Control \& Simulation division at Delft University of Technology, the Netherlands, has adopted the framework in order to support pilots (Borst, Sjer, Mulder, Van Paassen, \& Mulder, 2008; Borst, Mulder, \& Van Paassen, 2010; Dam, Mulder, \& Paassen, 2008) and air traffic controllers (Mercado, Mulder, \& Paassen, 2010).

The EID framework identifies three different but overlapping categories of events that the operators and designers of a complex system might encounter during operations (Vicente \& Rasmussen, 1992):

1. Familiar events,

2. Unfamiliar, but anticipated events, and

3. Unfamiliar and unanticipated events.

By its very definition, events in the last category could not have been anticipated by the designers of the system. As the consensus is that unanticipated events will always keep surfacing in complex systems, EID embraces this notion and instead tries to present the work domain - the ecology - in such a way that a skilled operator can better interpret information and use his or her cognitive capabilities to manage the unanticipated event.

As such, EID tries to "make visible the invisible" by visualizing the abstract properties of a process that are required to adequately control the system (Rasmussen \& Vicente, 1989).

Since EID is a framework, it is not supposed to provide an answer to the question of what the interface to be designed should look like. Instead, EID provides guidance on how to approach 
the problem of designing the interface. As such, it hands the designer two main 'tools' for realizing the task at hand: the abstraction hierarchy $(\mathrm{AH})$ providing a domain representation formalism, and the Skills, Rules, and Knowledge (SRK) taxonomy that captures how human operators deal with complexity. In other words, the $\mathrm{AH}$ is used to define what information needs to be presented, and the SRK taxonomy can provide clues on how information can be presented (Naikar, 2011).

\section{A-4-1 The Abstraction Hierarchy}

The abstraction hierarchy was developed as a means to represent the constraints of the work domain. The notion is that higher levels of abstraction relate to a system's purpose, whereas the lower levels disclose more information about the physical implementation details of the system. Links between elements are means-end links, enabling one to search the abstraction hierarchy from top to bottom in order to find issues and to get an understanding of the inner workings of a system, and traversing from bottom to top to obtain an increasingly wider view on the purpose of the system. Moving down in the AH therefore provides an answer to the question of how something is done, whereas moving up answers why something is done. Each level of the abstraction hierarchy provides a different view of the same system (Bisantz \& Vicente, 1994), and each level comes with a different set of constraints that affect the system's performance. The relation to EID comes from the notion that explicitly visualizing the constraints on different levels of the hierarchy should allow an operator to understand where contraints may be violated, thereby aiding in the analysis of unanticipated events.

There is a distinction to be made between the goals and the purpose of a system. Naikar et al. (Naikar, Hopcroft, \& Moylan, 2005) suggest that it is perhaps more appropriate to speak of the purpose of a system, as the abstraction hierarchy is supposed to be event-independent and instead seeks to describe the work domain itself. While the goal of an agent inside this domain may change over time due to the occurrence of a certain event, the purpose of a system generally does not.

An abstraction hierarchy for terrain awareness was developed by Borst et al. It is presented in Figure A-8 to demonstrate how an abstraction hierarchy can be mapped onto the domain of air transportation (Borst et al., 2008).

\section{A-4-2 Skills, Rules, and Knowledge}

Rasmussen's taxonomy of cognitive control describes three different levels of cognitive control (Rasmussen, 1983):

1. Skill-based behavior (SBB)

2. Rule-based behavior (RBB)

3. Knowledge-based behavior (KBB)

Operators employ skill-based behavior for simple, familiar tasks that require little to no mental effort, e.g., the simple tracking task of keeping the wings level while flying in mild turbulence. 


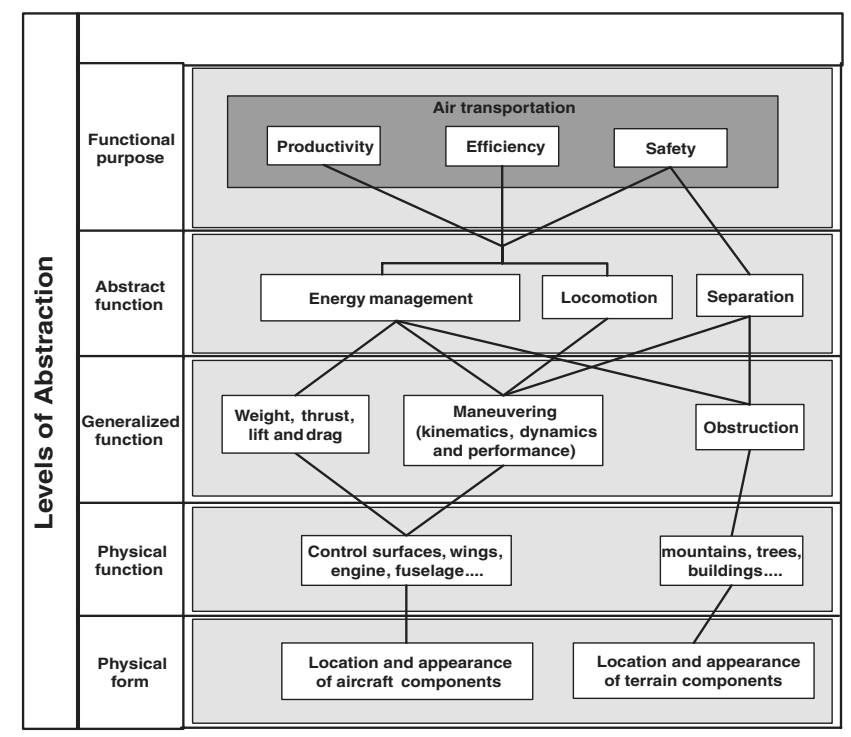

Figure A-8: Abstraction hierarchy for terrain awareness (Borst et al., 2008)

Rule-based behavior is employed when familiar solutions exist to familiar problems. For instance, when executing a standard turn, the aircraft's bank angle should be increased until the turn and slip indicator points out the predefined values. Knowledge-based behavior is triggered when neither skill nor rule-based behavior can solve a problem. For instance, when the standard rate turn can not be properly executed because the desired rate can not be achieved, a pilot will start to reason about what may have happened to the aircraft that is causing this observation: maybe the indicator is broken, or perhaps there is something wrong with the flight controls.

Employing knowledge-based behavior requires more cognitive effort than rule-based behavior, which in its turn takes more effort than skill-based behavior. Vicente and Rasmussen state that human controllers prefer to employ lower levels of cognitive control as these require less effort. However, which level is used depends on operator experience and familiarity with the situation at hand (Vicente \& Rasmussen, 1992). Complex tasks generally require the use of all three levels of cognition. The logical consequence of this is that 1) in order for an interface for a complex system to be effective, it should support the operator in using these three different levels of cognition as well and 2) if it is possible for an interface to transform a task such that it can be controlled using a lower level of cognition, it should do so. The latter aspect is an alternative way of stating that cognitive processing should not be forced to a higher level than necessary, explicating the opportunity to create rule-based shortcuts for KBB based efforts.

More concretely, the three principles of EID are directly tied to the three levels of cognitive control. These are listed below (Vicente \& Rasmussen, 1992):

1) SBB - To support interaction via time-space signals, the operator should be able to act directly on the display, and the structure of the displayed information should be isomorphic to the part-whole structure of movements.

2) RBB - Provide a consistent one-to-one mapping between the work domain 
constraints and the cues or signs provided by the interface.

3) KBB - Represent the work domain in the form of an abstraction hierarchy to serve as an externalized mental model that will support knowledge-based problem solving.

Skill, Rule, and Knowledge-based behavior can be identified by means of a Control Task Analysis, which attempts to describe the mental process of an operator as he tries to achieve a certain goal. The decision ladder is a tool that can be used to perform this analysis (Naikar, 2011). Contrary to the abstraction hierarchy, the concept of the decision ladder revolves around the notion of a specific goal (which is still subjected to the constraints of the work domain as laid out in the work domain analysis).

A representation of the decision ladder is shown in Figure A-9. In this figure, it can be seen how the human decision maker transitions from knowledge state to knowledge state. Also note the shaded areas that indicate how the SRK taxonomy ties in with the DL. In the DL model, it is assumed that this transition never occurs without an intermediate process. Important in the DL is that it recognizes that shortcuts can occur. This is exactly how training works; familiarization with procedures can cause an operator to skip past the knowledge-based decision making loop (see figure) and directly plan and execute instead. For instance, pilots learn during training that when they suffer a complete engine failure, the first thing to do is to attain the optimal glide angle (Borst et al., 2008).

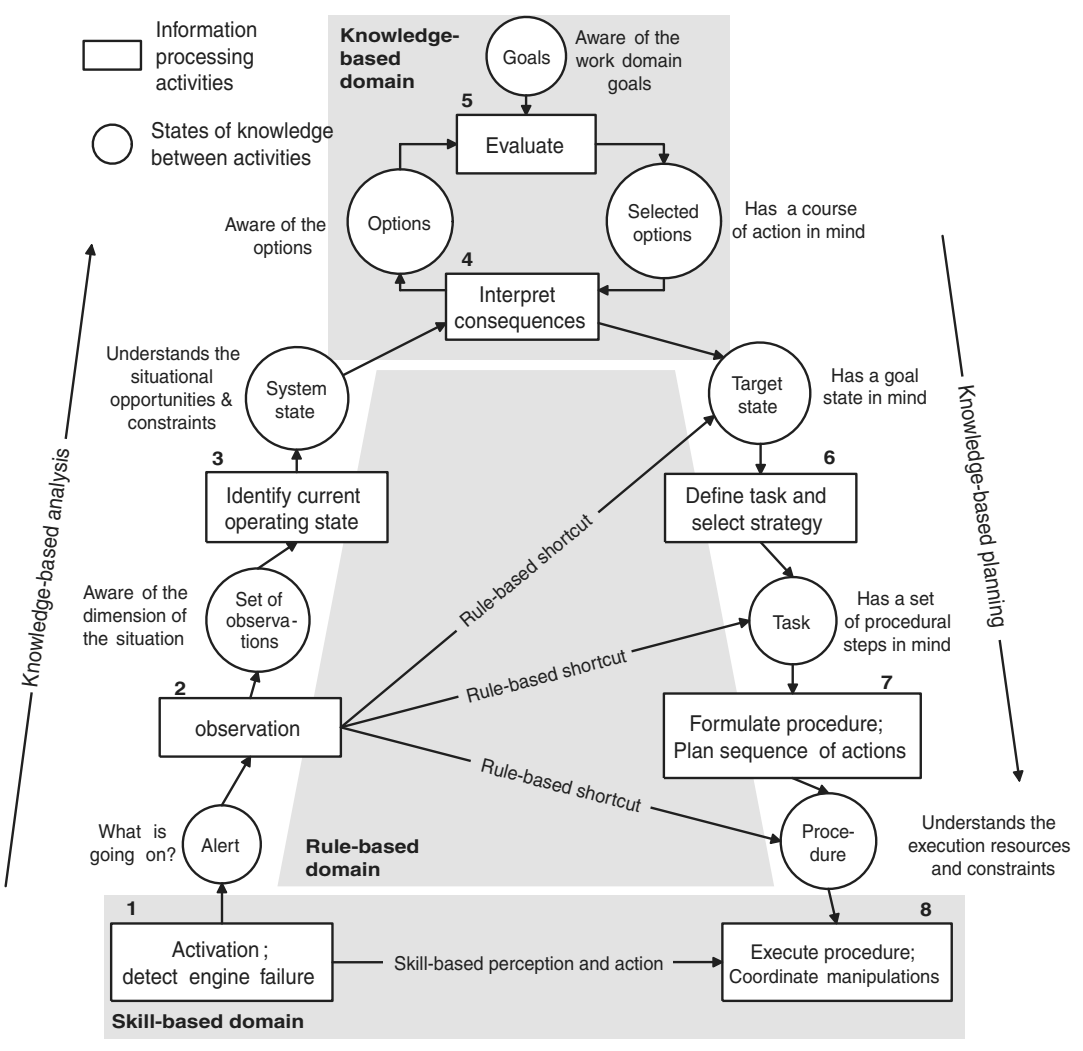

Figure A-9: The Decision Ladder (Borst et al., 2008) 


\section{A-5 A survey of existing emergency flight planning aids}

A number of emergency flight planning aids have been proposed. It should be noted that at the present time, none of these systems are commercially available. A common factor between all these aids, including the one to be developed as a result of this research, is that these rely on highly accurate fault detection and isolation modules in conjunction with sophisticated on-line flight envelope estimation modules. On-line flight envelope estimation is a subject of ongoing research, and the designers of emergency flight planning aids assume that eventually such modules will be available as an on-line data source for commercial flight applications.

\section{A-5-1 Emergency Flight Planner}

Research was conducted by Chen and Pritchett with the aim of investigating how pilots generate and follow a four-dimensional trajectory to a runway during emergencies (Chen \& Pritchett, 2001). Additionally, they examined what functionalities could support pilots in their decision making process and designed a prototype system in order to provide these functionalities. It was stated that at the time of writing, no cockpit aids existed for the purpose of generating emergency landing trajectories. At the time, the tools that flight crews would have available included charts and approach plates, which are mainly suitable for nominal flight conditions. Additionally, glass cockpit tools could provide flight path vectors and other related state-based information. However, these tools were found to be of limited use as these did not support planning or provide answers concerning which sets of actions would yield a desirable outcome.

They explicitly opted for a design aid that employed a procedure to convey information. Two reasons for this were the famliarity of pilots with flight path following procedures and because procedures are a common representation in tasks that feature a high workload in a complex environment. Another explicit design choice was the intention for the aid to be used in a separate planning phase and was intended to be used by the pilot not flying, thereby making the distinction between a planning and an execution phase. This choice was made in an effort to reduce the cognitive load on the pilots during the execution of the flight plan.

The Emergency Flight Planner (EFP) pilot interface is shown in Figure A-10. Note that pilots were expected to interact with the EFP through the Command Display Unit (CDU).

Failures were modeled by reconfiguring the model's aerodynamic coefficients. Stability and control contraints were modeled as limits on the pitch and bank angle, and the aircraft's velocity.

The minimum requirements to the interface were described as accepting input from the pilot and visualizing results such that a pilot can assess and execute the plan.

The visual component of the interface consisted of a plan view display based on the Boeing 747-400 Electronic Horizontal Situation Indicator (EHSI). It was decided not to combine the display with the actual EHSI as these screens are smaller and the authors were afraid of creating a cluttered interface. The visual interface also contained a vertical profile display for which multiple view modes were available. Additionally, the required aircraft state for every part of the path could be provided on a PFD-like display. A list of actions to be taken completed the interface. 


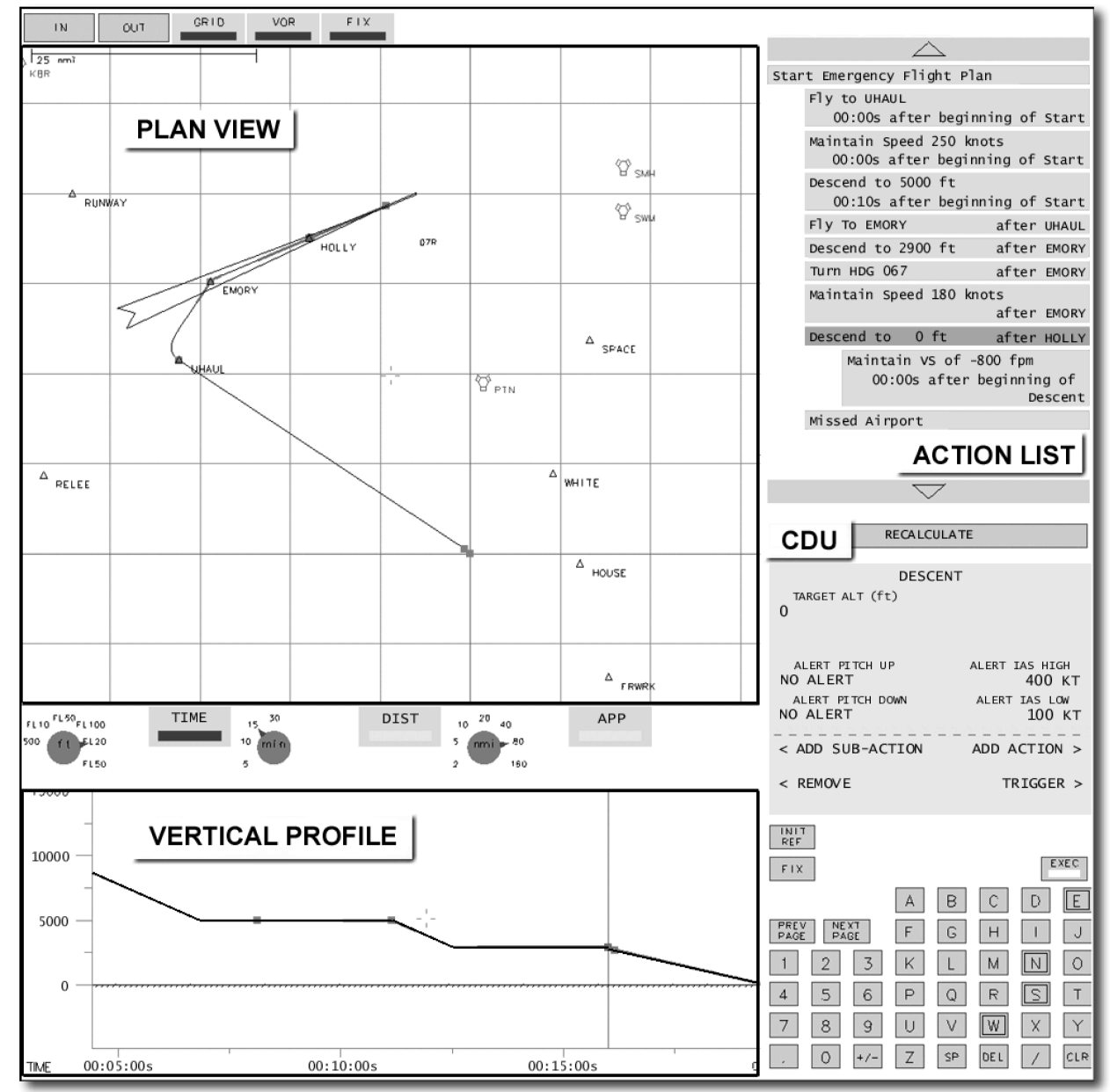

Figure A-10: The EFP pilot interface (Chen \& Pritchett, 2001)

Two alternative implementations were tested: an implementation in which pilots could build flight trajectories from scratch, and an implementation that was preloaded with suggested trajectories.

The results and conclusions drawn from an experiment with this display can be summarized as:

- With EFP Guidance, pilots engaged less often in aggresive rapid descent maneuvers.

- Interfacing with the EFP by means of the CDU was found to be cumbersome and, at times, confusing. Especially given the fact that the system needs to be operated in case of an emergency, a more streamlined control interface was deemed necessary.

- Significantly higher times to land were found when the EFP was used.

- When erroneous information was introduced, overreliant behavior was observed.

- The pilot's decision making process was found to be highly dependent on the type of malfunction. A tool that aids decision making should follow a similar rationale in order 
to properly support pilots, or otherwise pilots will disagree with the proposed plans, which nullify the usefulness of that tool.

\section{A-5-2 Emergency Landing Guidance System}

In 2008, Borst et al. published research that related to the development of an Emergency Landing Guidance System (ELGS) to support pilot terrain awareness with the goal of identifying terrain and selecting potential landing sites in the event of a total engine failure (Borst et al., 2008). The EID framework was used as a design approach.

The main motivation for the research was that there existed no system that supported the selection of and guidance to a landing location in the event of a total engine failure.

The chosen approach was to translate aircraft performance into energy based quantities relative to the terrain in order to define a 'glide mesh' that described the reachable and unreachable locations as projected onto the terrain. The result was a plan view display that mapped the excess energy onto the terrain. Suitable landing locations, defined as relatively smooth and flat areas, were also identified on the display. Both a conceptual representation and the actual Terrain Awareness Display can be seen in Figure A-11.
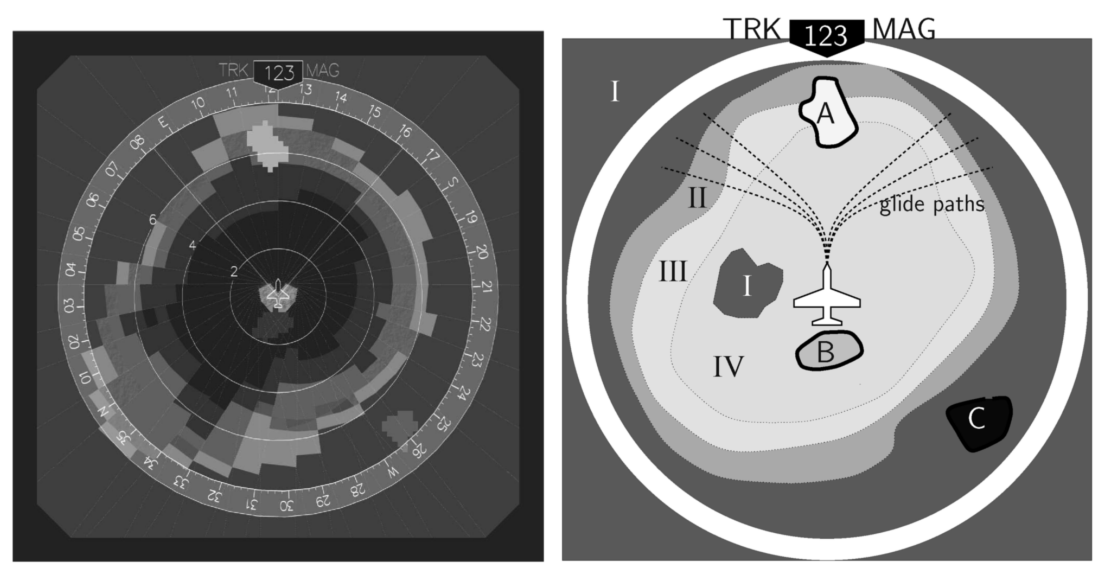

Figure A-11: Terrain Awareness Display, conceptual (right) and actual representation (Borst et al., 2008)

In an experiment, performance, situation awareness, workload, and safety data were gathered. In terms of performance, landing location selection and touchdown position were scored.

The main conclusions of the research can be summarized as:

- Higher levels of situation awareness (projection and metacognition, i.e., the ability to predict future states and assess one's own situation awareness, respectively) of pilots were significantly improved.

- Pilots made better landing location choices with the ELGS active.

- Pilots navigated closer to terrain, implying reduced safety. An argument was made that this behavior was observed because the ELGS enabled pilots to behave in this way and 
that operating closer to terrain is not necessarily less safe given the improved situation awareness.

It should be emphasized that, as opposed to other planning aids described here, that the ELGS did not specifically consider the case of landing on a runway.

\section{A-5-3 Automated Path Planning Aid}

In 2012, an Automated (Path) Planning Aid (APA) was developed by Watts et al. (Watts, Claus Christmann, Johnson, Feigh, \& Tsiotras, 2012). The research consisted of a human factors study regarding the development of this aid. The emphasis was placed on the aspects related to the human interface design. The task that was considered was selection of a suitable emergency landing site and subsequent formulation of an "expedient and safe trajectory".

The aid was built with the understanding that it would be operated by the pilot not flying. As such, an experiment was set up in a limited simulation environment where the pilot not flying was played back an emergency diagnosis from the pilot flying. With knowledge of the emergency, the pilot not flying was supposed to use the APA to find and select a suitable landing area. The APA provided ratings for every landing site, which were provided by an expert.

Both familiar and unfamiliar emergency scenarios were evaluated in different flight phases, i.e., the takeoff, cruise, and landing phase.

A visual representation can be seen in Figure A-12. A modified Navigation Display Control Panel was implemented using a touch screen interface and served as the main input source for the pilot (Figure A-13). The APA also made use of the CDU to interact with the pilots. It seems that in this research, every airport was given one suggested flight path and the aid did not consider different runways on one given airport, thereby focusing on flight regimes where the airport selection is dominant over the exact landing condition. It was mentioned that aspects such as runway length were considered in the aid's rating metrics.

One of the outcomes of the experiment was that it was observed that pilots made faster selections in familiar scenarios as opposed to unfamiliar scenarios. It was found that there was no single way in which every of the eight pilots operated the display, as it was stated that "each participant used his own method to make the APA most useful". Participants indicated that the most useful features were the ability to filter out unnecessary information, (i.e. suboptimal trajectories) and the ability to get extended information on the landing sites.

\section{A-5-4 Emergency Landing Planner}

An Emergency Landing Planning (ELP) algorithm was developed by Meuleau et al. for the purpose of landing damaged aircraft (Meuleau, Plaunt, Smith, \& Smith, 2009). The approach taken was to score a generated trajectory based on the associated risk imposed by distance to the landing site, en route weather, path geometry, ceiling, visibility and winds, instrument approaches available, danger to the population along the flight path and runway properties and services available (Meuleau et al., 2009, 2011). The path planning algorithm was based on Hybrid $\mathrm{A}^{*}$, which is a well known (graph-based) planning algorithm. The initial research 


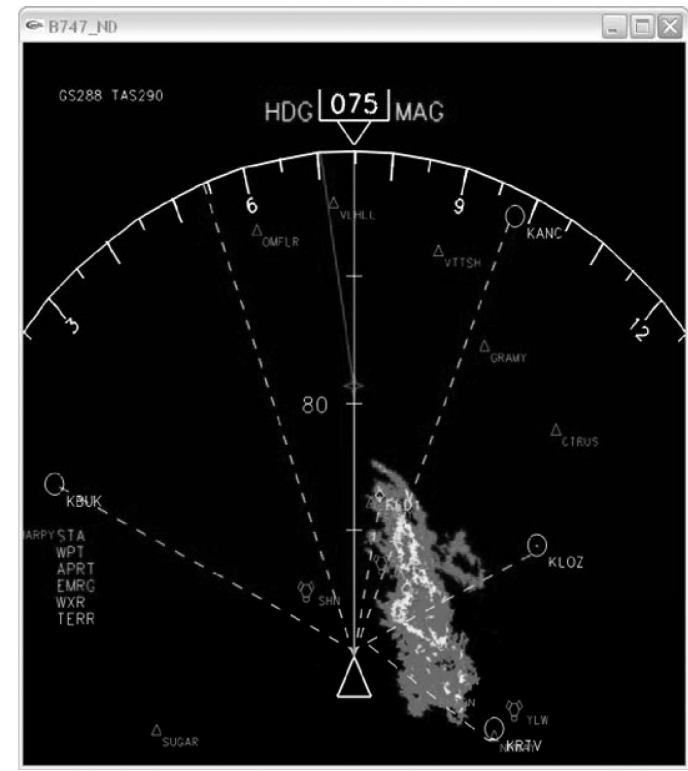

Figure A-12: Navigation Display of the APA with suggested routes represented as dashed lines

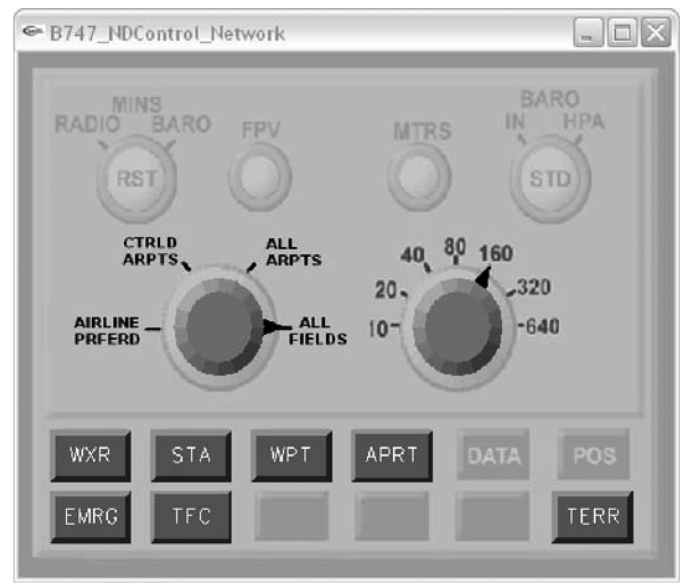

Figure A-13: Modified Navigation Display Control Panel of the APA

described that the planning algorithm was not optimal due to the predetermined placement of the graph vertices, which limited the amount of ways obstacles could be avoided. It was also described that the algorithm ignored aircraft dynamics to a large extent. Later on the planner was modified to include a 'trajectory planner' module, which would verify that a (sub)trajectory calculated by the algorithm would indeed fall within the safe flight envelope of the aircraft.

The planner was subsequently integrated into the avionics systems of a flight simulator, where a pilot would interface with the planner by means of the CDU. The planner output was visualized on the ND. One representation of the ND with the planner active can be seen in Figure A-14.

In an experiment executed in a 6 DOF flight simulator, crews of two were tasked with performing an emergency landing using either the ELP, a basic list of nearby airports and their characteristics, or an intermediate aid that performed a partial evaluation of the risk associated with each runway. An adaptive controller was used to facilitate the task of controlling the aircraft. Because previous research had shown that crew awareness of the state of the control surfaces was reduced in case of adaptive control, an additional display was used to indicate their state (see Figure A-16). In order to visualize the current flight envelope limits, a modified PFD was used that indicated limits on speed and roll (Figure A-15).

Five teams of professional pilots participated in the experiment. It was found that the pilots generally chose to land on the runway that was rated highest by the ELP. In some cases, evaluation of the possible flight plans took too long, which resulted in a new set of plans that had to be evaluated. In general, decisions were made faster when the ELP was used. The ELP outperformed the other options mainly when weather was severe, because many different flight plans would have to be evaluated at a time. 


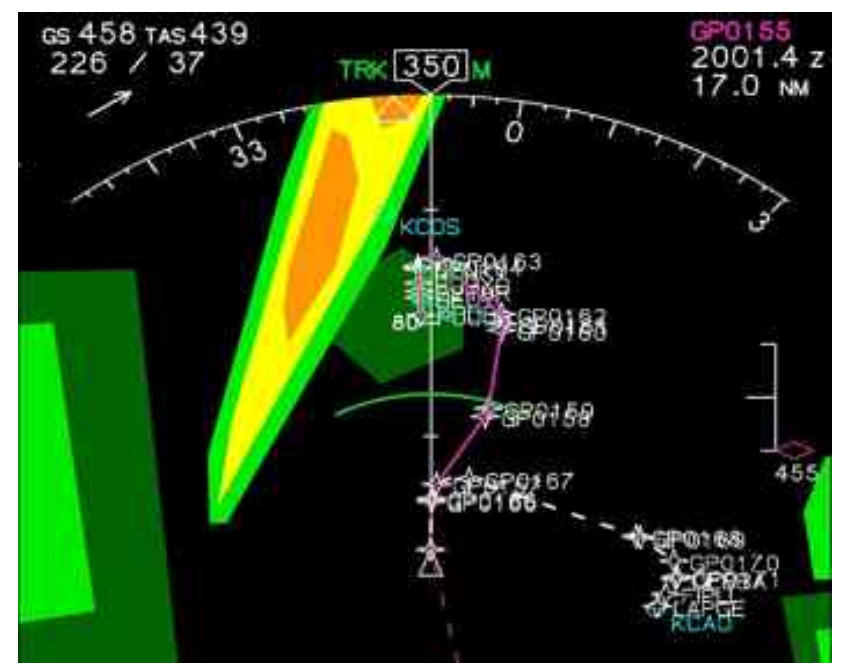

Figure A-14: Navigation Display implementation of the ELP, showing the current flight plan (magenta) and an emergency trajectory (dashed white) (Meuleau et al., 2011)

The most interesting result from the experiment is that damage severity was not a factor: while pilots always preferred to have the ELP available as they felt it reduced workload, the damage severity did not make the pilots more likely to adhere to the presented flight plan. It was mentioned that "a direct route to a point about 10 miles out on the final approach was appropriate and was relatively easy for them to construct" (Meuleau et al., 2011). The authors speculate that this was due to the use of the adaptive controller as it facilitated the task of flying so much that the crew had enough time to plan a trajectory by themselves. It was mentioned that without the controller, pilots would probably not have had the time to consider multiple trajectories or to plan a path manually.

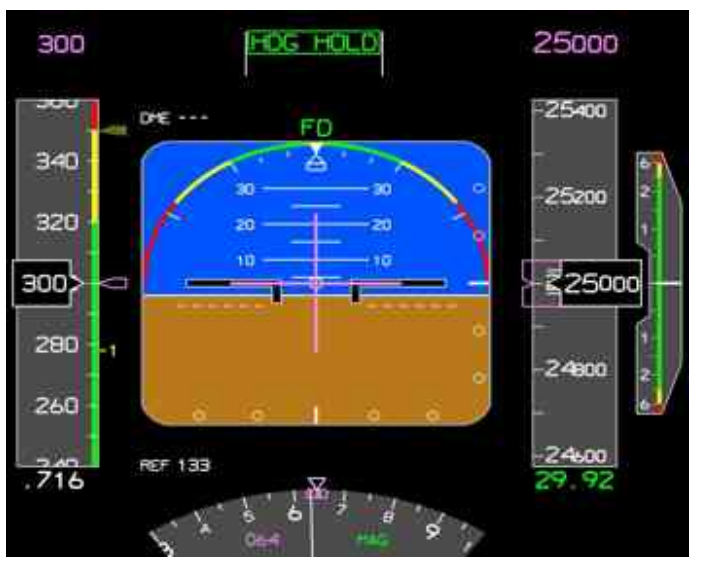

Figure A-15: PFD with flight envelope limits visualized (Meuleau et al., 2011)

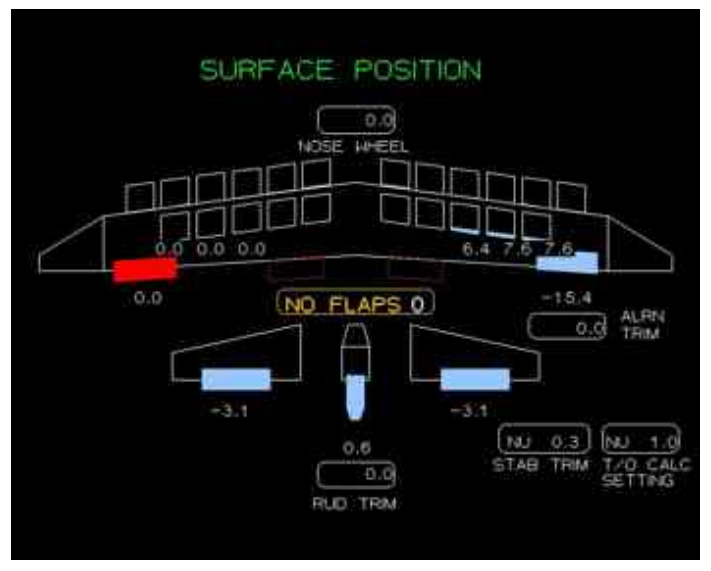

Figure A-16: Auxiliary interface displaying damage and control surface deflections and saturations (Meuleau et al., 2011) 


\section{A-5-5 Discussion}

A number of interesting lessons can be learned from this brief survey. The most important aspect may very well be that there is a very broad spectrum of events that can occur that require an emergency response. Events requiring an emergency response can be categorized in performance altering and non-performance altering, and pilots have been observed to show different behavior in these conditions (Chen \& Pritchett, 2001). This is just one categorization, though. Depending on the exact cause of the emergency, landing will be either more or less urgent and flying will need to proceed with more or less caution. These parameters influence first of all the destination and second of all the chosen trajectory. When the discrepancy between a suggested plan and the plan a pilot has in mind is too large, the suggested plan may be discarded. In the experiments that pilots follow a trajectory, this has indeed happened (Chen \& Pritchett, 2001; Meuleau et al., 2011). This means that there is a need for planning algorithms to capture, reason with, and display all - or more - information pilots have available when making decisions.

Second of all, planning takes time. Generating plans automatically may save time, but this may not always be the case. In the performed experiments it was observed that viewing the available candidates and interfacing with the aids in general did not always save time. It was generally observed that the CDU was not a very suitable candidate for interfacing with an emergency planning aid (Chen \& Pritchett, 2001; Meuleau et al., 2011; Watts et al., 2012). It is important that the required planning time is reduced to a minimum because there may be hard time constraints, but also because a window of opportunity for navigating to a specific place may be closing while planning.

When introducing faulty information, overreliance was also observed in pilots (Chen \& Pritchett, 2001). This is a recurring theme in automation and this is one of the reasons why EID can be effective as a design strategy. Especially when algorithm complexity increases and its inner workings become less clear to a flight crew, it becomes progressively harder to decide whether to accept or reject a proposed solution.

A difference can be observed between the EFP and ELP on the one hand, and the APA on the other. The former two did include a PFD that represented flight envelope limits. This would enable a pilot, at least from trajectory segment to trajectory segment, whether or not the segment would indeed be flyable. The APA lacked such a visualization, perhaps because this was not a consideration in the experiment as the pilots were not expected to fly on of the suggested paths, but just to commit to one of them.

Finally, replanning may be required because of the occurrence of new events such as an additional failure. This may imply that the best candidate trajectory may suddenly become less feasable or infeasible. If replanning is not supported in an effective way, additional time may be lost. Approaching the planning problem in terms of reachable and not-reachable space as was done in the ELGS may be an interesting alternative. 


\section{Appendix B}

\section{Model Properties}

The model was created by performing the following consecutive steps:

1. Create a set of trim points for the given configuration (see Table B-1)

2. Linearize the model at the trim points

3. Simplify the dynamics, retaining only the roll subsidence eigenmode and assuming only the inboard ailerons are available for controlling the aircraft's roll angle

The configuration that was used to linearize the model is shown in Table B-1.

Figure B-1 shows how the velocity affects the parameters $K_{p}$ and $\tau_{p}$. Figure B-2 shows this effect in terms of an aileron step input on the roll rate.

For the airspeed value of VTAS=170 kts, the responses of the nonlinear, linearized, and simplified linearized model along with its DUECA implementation are shown in Figure B3. It can be observed that especially with respect to the roll rate behavior the shift from the linearized model to the simplified linear model is quite an oversimplification. However, this effect does not seem as pronounced with respect to the more integrated states on the

Table B-1: Configuration of RECOVER model

\begin{tabular}{lrl}
\hline Parameter & Value & \\
\hline Mass & 200,000 & $\mathrm{~kg}$ \\
Failure mode & 0 (none) & \\
Gear & 1 (down) & \\
Flaps & 20 & $\circ$ \\
Altitude & 2000 & $\mathrm{ft}$ \\
Speed & $150-200$ & $\mathrm{kts}$ \\
Flight path angle & 0 & $\circ$ \\
\hline
\end{tabular}



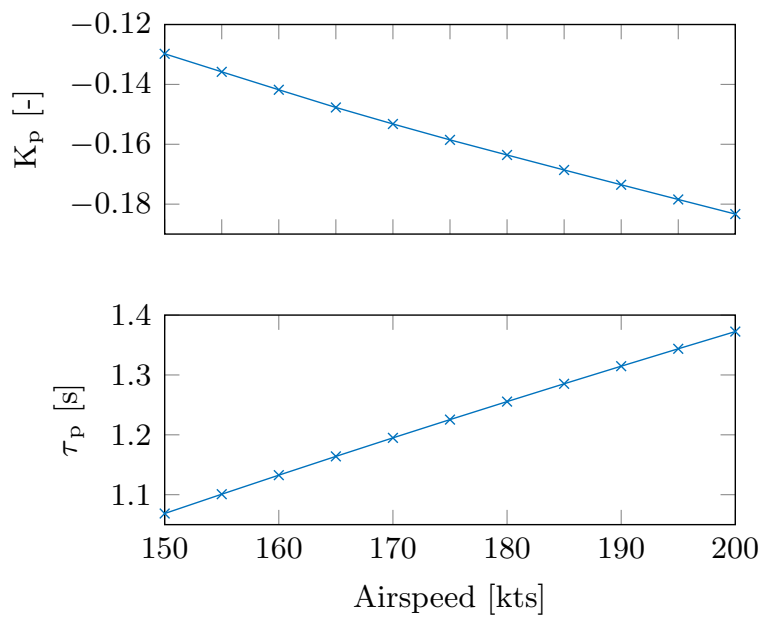

Figure B-1: Speed-dependent parameters for the model

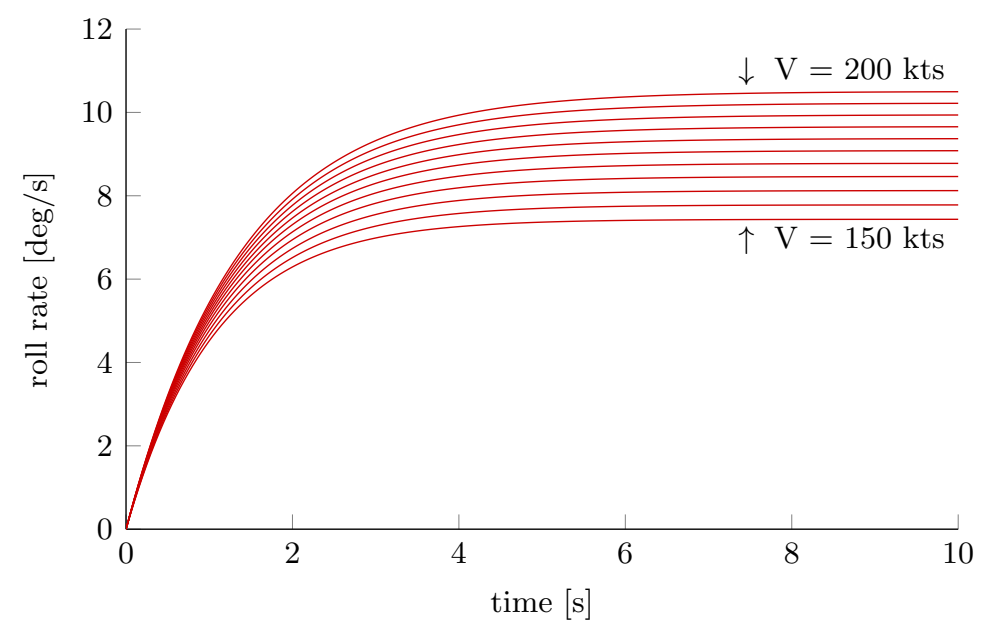

Figure B-2: Comparison of the step responses of the simplified model for a range of airspeeds

time scale shown. The response of the DUECA implementation which uses a Forward Euler integration scheme is a very close match to the MATLAB implementation of the model.

In the accompanying paper, the choice for a model with simplified dynamics was justified from the perspective of fault-tolerant control; an effective fault-tolerant (roll rate) controller is expected to simplify the control task in a manner that is similar to the simplified behavior shown here. The responses shown here lend credibility to the idea that the roll dynamics used in the experiment bare resemblance to a 747-type aircraft - in the off-nominal configuration described in the paper - with a fault-tolerant rate controller present.

It is therefore believed that in terms of the primary focus of this study, i.e., aiding pilots in estimating reachable navigation states, the simplifications made can be justified. 

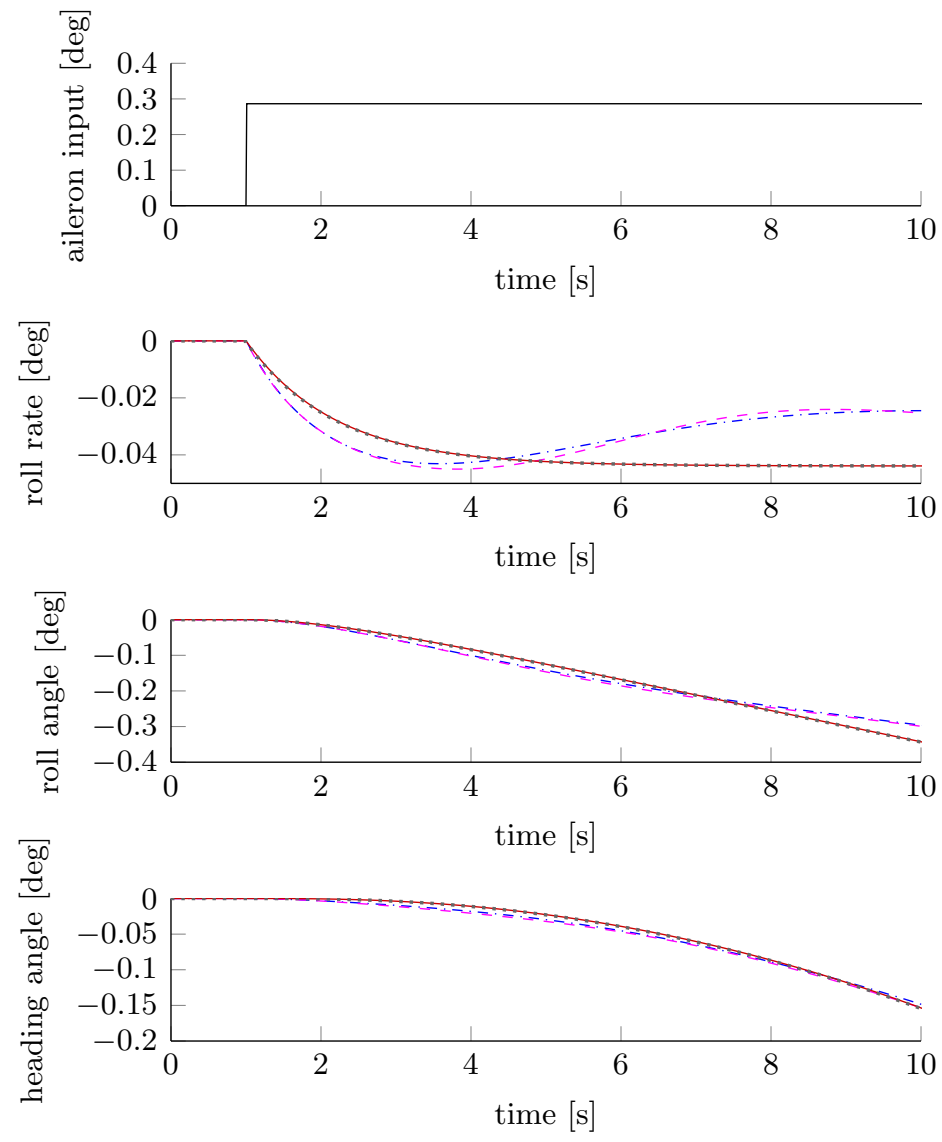

- - Nonlinear model $-\ldots$ Linearized model
— Simplified linear ...... DUECA simplified

Figure B-3: Comparison of the step responses of the various models 


\section{Appendix C}

\section{DUECA Simulation Architecture}

\section{Essential information}

- DUECA project name: FlightEnvelopeVisualization

- Tested on: Mac OSX 10.11 El Capitan (DUECA V2) and SIMONA (DUECA V1)

- Additional libraries: HMIlib (for EFIS displays), SDL2 (for OSX joystick support), GTK2 (for ECI), GTKMM, AirportDB (for ILS, ND, outside visual), TinyXML (for AirportDB) (see Makefile)

- Primary contacts: Tom Rijndorp, Olaf Stroosma

\section{Overview of Modules}

Figure C-1 lists the different modules that exist in the project. Also shown in the diagram are the DUECA channels that are used to pass information between the modules.

Most DUECA modules are simple facade classes that read and write DUECA channel data. The actual calculations and visualizations are typically done in a class that is being instantiated by the module, e.g., the DUECA module PFDModule passes information to B747PFDModule which takes care of showing the PFD. This creates modularity by removing DUECA dependencies. For instance, a simple command-line application navEnvCalc was written that calculates the Navigation Envelope (see paper) and writes the results to a text file, using only the FEVModel class inside the vis-model folder.

The remainder of this appendix will briefly introduce the classes that are being used in the simulation environment. 


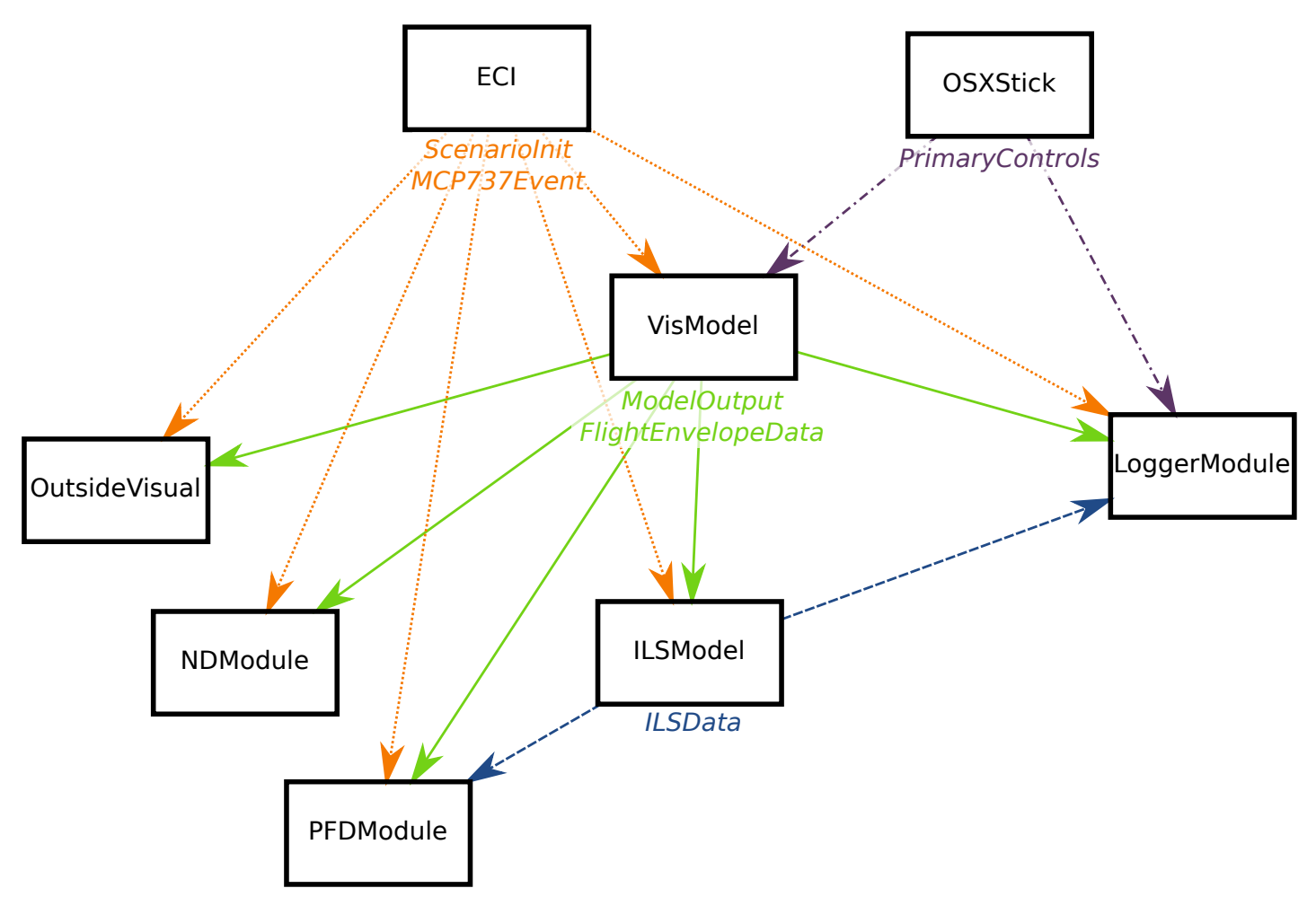

Figure C-1: Interaction between the different DUECA simulation modules along with channel names

ECI Experiment Control Interface (ECI) designed in Glade. Can send preconfigured experimental conditions (using JSON files) to the other modules when the simulation is in "hold current" mode. Alternatively, model and display configuration can be edited directly from the ECI. The ECI is shown in Figure C-2.

OSXStick The simulation experiment was developed on Mac OSX. This class provides basic minimal joystick support using the SDL library (you will need to install and compile this library yourself). When actice, the module searches for connected joysticks and connects to the first one it finds. At least the Logitech Wingman Extreme 3D is supported. Not tested with multiple joysticks connected.

VisModel This module contains the dynamical model. Additionally, it calculates the flight envelope data that feeds the interfaces. This module is a facade class for the FEVModel class that runs the actual model.

ILSModel Calculates localizer error using the scenario information (for the airport/runway configuration and selected runway) and the dynamical model output using simple trigonometry. This module uses Clark Borst's AirportDB class to read airport configurations stored in run/run-data/. 


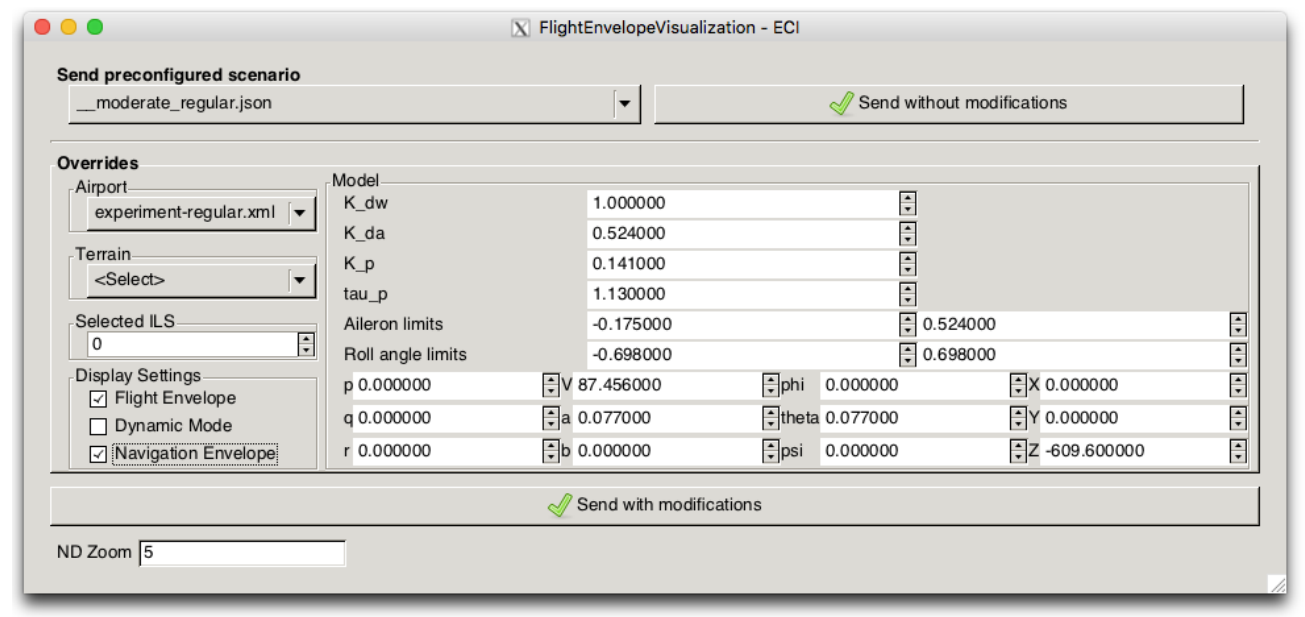

Figure C-2: Experiment Control Interface

PFDModule Boeing-style PFD implementation. Developed by Joost Ellerbroek, modified to incorporate the PFD interface additions described in this document.

NDModule Boeing-style ND implementation. Developed by Joost Ellerbroek, modified to incorporate the ND interface addition described in this document.

OutsideVisual Simple outside visual using both Clark Borst's AirportDB (for visualizing runways) and AC3DLoader (for visualizing scenery).

LoggerModule Module that logs simulation data and stores it into ASCII text files. Internally based on Herman Damveld's LoggerInterface class. 


\section{Appendix D}

\section{RECOVER Trim Database Documentation}

In the earlier stages of this study, an attempt was made to directly work with the GARTEUR RECOVER model. The general idea was to build a trim database out of sets of feasible trim states, using the specific "El Al" failure mode, and to design navigation state representations based primarily on trim state information, placing an emphasis on the slower dynamics and larger preview times. Unfortunately, it turned out that the process of defining a trim database was problematic as a consequence of the model and especially the failure mode definition. However, a significant effort was made to automate the process of calculating trim states, storing these in a database, and performing elementary operations (e.g. selection) on the data.

For students interested in continuing down this road of building a trim database for the RECOVER model, it may be advisable to look into employing different trim routines or analyzing a different failure mode than the $\mathrm{El} \mathrm{Al}$ failure mode. Either way, if one would want to calculate families of trim states, it may be a good idea to read through this document and work with the accompanying MATLAB code if applicable. At the very least this document will provide some pointers as to how a trim database could be constructed based on the RECOVER (Simulink) model and help in understanding the potential challenges in terms of performing batch operations on the model.

\section{D-1 Compatibility Notes}

This document describes the RECOVER model version v2.3_Matlab R13SP1. This model was confirmed to work flawlessly (i.e. the model compiles and runs) with MATLAB R2015b on Mac OS X 10.11 El Capitan. Running the Simulink model will trigger some warnings unless the code snippet below is executed before evaluating the model.

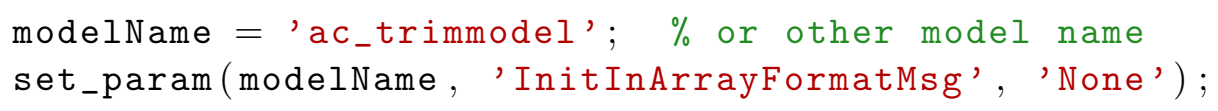




\section{D-2 Background}

RECOVER consists of a MATLAB interface and a Simulink model, where it should be noted that almost all model data is stored in several Simulink .mdl files ${ }^{1}$. Perhaps to most useful resource to get a better understanding of RECOVER is the documentation of Delft University Aircraft Simulation Model and Analysis Tool (DASMAT), as RECOVER is implemented in this framework (Linden, 1996).

It may be suitable to directly work with the functionality as it was provided in the release. In order to do this, only a small number of functions need to be called. For this, see Table D-1.

Table D-1: Functionality and corresponding $m$-files

\begin{tabular}{ll}
\hline Action & Corresponding m-file \\
\hline Initialize model parameters & ac_init.m \\
Initialize autopilot parameters & init_auto.m \\
Select failure mode & select_failure.m \\
Trim the model & trim_ac.m \\
Trim cost function & trimcost.m \\
\hline
\end{tabular}

When running or inspecting these files, note that these are pure scripts; as a consequence, all defined variables will end up in the base work space. This was most likely done because Simulink / MATLAB interaction is most easily done by sharing the base workspace. Unfortunately, this practice pollutes the base workspace, especially since structs were never used to reduce the amount or improve the structure of the variables.

\section{D-3 Non-interactive Automation}

Running trim_ac.m, the user is presented with an 'interactive' MATLAB command-line interface that will request input variables in an interactive fashion. Once all the required information has been entered, the model is configured. Then, the trim routine will run and the script will save the result in the base workspace and ask if this single trim point should be saved to disk ${ }^{2}$.

Obviously, this approach does not work for calculating large sets of trim families. Therefore, code was written to simplify this process.

Word of caution: As the RECOVER model was abandoned about midway through the thesis project, the code is in a state where I'd rather not hand it off to anybody; if you read on or check my code, you will be confronted with some hardcoded values, sparse comments, and other things that will significantly reduce your appetite to carry on. However, I can guarantee that going through this yourself from the start will be worse. While RECOVER is

\footnotetext{
${ }^{1}$ If you are to modify and save a file, you may have to save the file as .slx file instead. This is a binary format, whereas the older Simulink .mdl files are stored as text.

${ }^{2}$ Note that DASMAT has defined several file extensions: .tri for trim states, .inp for input sequences, and more. All of these files are actually plain .mat files with a different file extension. This may cause MATLAB to reject loading the data inside the file. If so, just rename the file.
} 
very significant from an engineering perspective, from a code perspective it is atrocious and interacting with it is a pain. If you are looking to automate trimming this model in some way, I would suggest to see my work as a starting point and not as a ready-made project. With that said, my additions are in the trim folder. These are described below.

\section{D-3-1 Function createTrimDatabase()}

This function contains the code to calculate a trim database. This function should really be modified to accept a struct with the desired configuration, but this is easily done. This function is essentially a wrapper for trim_ac.m: it takes a predefined set of trim states and executes trim_ac.m for every state. Except that it does not call trim_ac.m, as this script requires keyboard interaction that is unfortunately not easily bypassed. As a result, this function includes the actual code of trim_ac.m, while stripping all user interaction and substituting the preconfigured values.

Note: It will take a bit of an effort to make this function suited for all flight conditions. These are listed in Table D-2. As the only interest at the time of this study was trimming turning flight states, only flight condition 3 is implemented in this function. Do note that contrary to the name given, it is actually possible to trim at any given flight path angle. If, however, one is interested in trimming in straight and level flight only, flight condition 1 was implemented in createTrimDatabaseLevelStraight () for this purpose. This code was also used to calculate the trim points that were used in Appendix B.

Table D-2: Functionality and corresponding m-files

\begin{tabular}{ll}
\hline Flight Condition ID & Trim method \\
\hline 1 & straight-and-level trim \\
2 & pushover-pullup \\
3 & level turn \\
4 & thrust-stabilized turn \\
5 & beta trim \\
6 & specific power turn \\
\hline
\end{tabular}

For every trim point that is calculated, the trim variables are placed as fields in a struct, and stored in a .mat file, along with the result of the cost function, the aircraft configuration, and whether the engine could be trimmed for this state or not.

Note that as trimming takes up to a minute for one state, the process of calculating many trim points takes time. However, you can stop the process at any point; when you run the function again with the same parameters, the function will check which trim states already exist on disk (a new file is created for every trim point) and pass over these (notice the definitions of trimStateExists() and trimConfigurationsEqual() in createTrimDatabase.m as these may need to be modified, depending on future implementation).

\section{D-3-2 Class TrimDatabase()}

So far, createTrimDatabase() resulted in a folder full of trim states. How should these files be operated on? This is why TrimDatabase.m was written. It is a MATLAB class that 
encapsulates functionality for performing actions on the database. Most importantly, it can load the .mat files that are stored by createTrimDatabase(). Instructions for loading a previously created trim database:

$\mathrm{TDB}=$ createTrimDatabase ('path/to/mat/files/');

TDB. load ();

Under the hood, this class calculates properties (index vectors, really) that allow the user to quickly sort through the database by different parameters. For instance, one could call

trimset $=$ TDB.filterTrimPoints ('gear', true);

to obtain all trim points where the gear is down. The result of these filtering operations is a vector of TrimPoint objects.

\section{D-3-3 Class TrimPoint}

TrimPoint is a simple class that aims to simplify working with trim data. This class is used by TrimDatabase for storing its data. Furthermore, it can create a new TrimPoint from the structs that are stored by createTrimDatabase(). The class defines 'getters' for obtaining the trim state information. For instance, calling myTrimPoint.d_e yields the elevator deflection, myTrimPoint. $\mathrm{V}$ yields the airspeed, and myTrimPoint.mass yields the mass at which the trim point was defined.

\section{D-4 Known Issues with Trimming RECOVER}

- The main concern is that there are undocumented issues with the trim routines. Also, aspects of the trim routine itself are not documented at all, leaving the users guessing as to what some variables or calculations represent. For flight condition 3 (the steady turn), the code from trim_ac.m was compared against the documentation from NASA's LINEAR program (Antoniewicz, Duke, \& Patterson, 1988), and it was found that some calculations deviate. The RECOVER trim implementation crashes at load factors close to 1.0. Replacing the (undocumented) RECOVER trim equations with the LINEAR equations solves this problem. While there must have been reasons for the original implementation, as these are undocumented, it is unclear why these equations are different and what they represent.

- The built-in trim routine trim_ac.m does not make a distinction between left and right banking turns, which is an issue in case of failures resulting in an asymmetric flight envelope such as the ' $\mathrm{El} \mathrm{Al}$ ' failure mode. This can be corrected by implementing equations from LINEAR.

- It was found that especially for the 'El Al' failure mode, very few states can actually be trimmed, even when it should be expected that a state should indeed be a trim state ${ }^{3}$.

\footnotetext{
${ }^{3}$ In many cases, states between two trim states can not obtain a sufficiently low cost function value (i.e. the accelerations are not zero for this state). The DASMAT documentation recommends to consider a state with a cost function smaller than $10^{-10}$ as a trim state, which seems reasonable.
} 
One hypothesis (that was not verified) is that this occurs due to the use of lookup tables in the Simulink diagram, which does not necessarily produce smooth derivatives that are suitable for running optimization algorithms against. If this is indeed the case, it may not be possible to create a trim database at all for this model. The issue described here also manifests itself for the failure-free configuration, but to a far lesser extent. 


\section{Appendix E}

\section{Justification for a Single Pilot Operated Experiment}

The experiment was performed with a single pilot at a time, as opposed to performing the experiment using a flight crew consisting of both a captain and a first officer. Below follows a justification for this design choice.

One of the topics of interest in the aviation industry is the possibility of transitioning to singlepilot operations, i.e. operating under conditions that currently require two or more pilots. The largest motivator for this transition are potential cost savings (Comerford, Brandt, \& Mogford, 2013). Over the course of a technical interchange meeting at NASA Ames Research Center, it became apparent that more enabling technologies and decision support tools are necessary. It was also mentioned that there is a particular concern about off-nominal circumstances, as these significantly increase the mental workload (Comerford et al., 2013). Allocating this workload to a single pilot may be unsafe given the current flight deck design.

Various possibilities exist with respect to the re-allocation of tasks and responsibilities of the second pilot, such as delegating these to a ground-based support team that oversees multiple flights at the same time. Another logical option is replacing the second pilot by more capable avionics systems (Comerford et al., 2013). It has been stated that single-flight operations are generally desired when critical issues - safety - can be accounted for (Schutte et al., 2007).

The interface that is being developed may be one of these systems that can improve the capabilities of a single pilot by providing him or her with more integrated information. It is hypothesized that this will to some extent reduce the workload experienced by the pilot. As this is exactly in line with the need for a reduction in workload during off-nominal circumstances, it was decided to execute this experiment as a single-pilot operation. 


\section{Appendix F}

\section{Experiment Briefing}

The following briefing was read out to the participant by the researcher. The end of the briefing contains screen shots of the interface adjustments (see Figure F-1). Explanations on all interface elements were given until the researcher was confident that the participant had a deep enough understanding of the information presented to continue to the training phase. 
You are going to be flying a number of localizer intercepts in our SIMONA research flight simulator using a 747-like aircraft.

"Unfortunately", shortly after takeoff, your aircraft sustained damage and as a result, you would like to land as quickly as possible on a suitable runway.

\section{Structure}

In order to gather quantifiable data and to make good effective use of your time, this scenario will be simulated as follows:

\section{Familiarization}

You will first be given the opportunity to make yourself familiar with the failure case. During this phase of flight, your only task will be to get an understanding of the failure case. This will last a few minutes.

\section{Align with runway}

Then, your aircraft will be positioned in the vicinity of an airport. Initially, two localizers will be visible and you will be asked to explain on which runway you would like to land. Regardless of your choice, a predetermined runway will be selected for you, which is either a more appropriate, less appropriate, or similar choice. The corresponding ILS will be activated and you will fly the scenario. It will be your task to intercept the localizer at or before the first waypoint indicated on the ND both as timely as possible (because your aircraft sustained unknown damage) and as far ahead as possible (because you will need to prepare for a stable landing), or whatever combination of these two factors you see fit given the situation. A second marker is placed where the glide slope intercepts your current altitude. The simulation will be halted when you pass this second marker.

\section{Limitations}

You are not to exceed the visualized bank limits. If you do, the aircraft will stall and simulation will be halted. The scenario will then be flown from the beginning.

\section{Repetition}

When the scenario is completed, you will be flying a similar scenario (i.e. another round of familiarization and localizer intercept) with a different configuration. 


\section{Aircraft Model Properties}

The aircraft has been modeled with an aileron failure, the specifics of which are for you to identify. Please note that the specifics of this failure will change from one scenario to the next! In order to facilitate the flying task (and in order to constrain the experiment), the model is fitted with an autothrottle, the reference speed of which will be fixed. A yaw damper is present, too. Furthermore, the autopilot mode will be fixed to altitude hold, i.e., all stages of flight in this experiment will be horizontal.

The aircraft is preconfigured in an approach configuration, which is also fixed.

\section{Flight Deck Interface}

- You will be flying the aircraft with a side stick only. This side stick will only respond to lateral inputs.

During the experiment, you will fly the aircraft using three different interface combinations:

\section{Baseline}

This flight deck interface will present you with a combination of a basic Boeing-style PFD and ND. Differences with respect to the standard PFD you are comfortable with may be:

- bank angle limits are visualized by means of a red 'barber pole' on the bank angle scale.

Differences with respect to the standard ND you are comfortable with may be:

- Runways are visible. The runway with the selected ILS is depicted in magenta, others are gray. Additionally, a vector is drawn from the selected runway threshold outward, representing the localizer that is to be intercepted. Two markers are also visible on this line, the one furthest away from the runway representing where the localizer should be intercepted and the nearest one representing where the glide slope intersects your current altitude.

- A trend vector representing your predicted flight path with a preview time of 30 seconds is visible.

\section{PFD Additions}

The PFD will be augmented with additional symbology.

- Attainable roll rates presented as a five-second bank angle, as well as the current roll rate, are visualized on the bank angle scale. Examples will be provided below.

- Symbols indicating the maximum bank angles you can reach from your current angle within a 10 -second time window.

- An additional symbol, from now on referred to as roll-out indicator (ROI) will be visible on the bank angle scale. 


\section{PFD + ND Additions}

Besides the additions under (2), the following modification is present on the ND:

- A fan-shaped interface element representing a preview of all possible future states for the next 60 seconds will be visible. From now on, we will call this the 'navigation envelope' (NE). This shape represents all the possible combinations of heading and position your aircraft can attain over the preview time. Examples will be provided below.
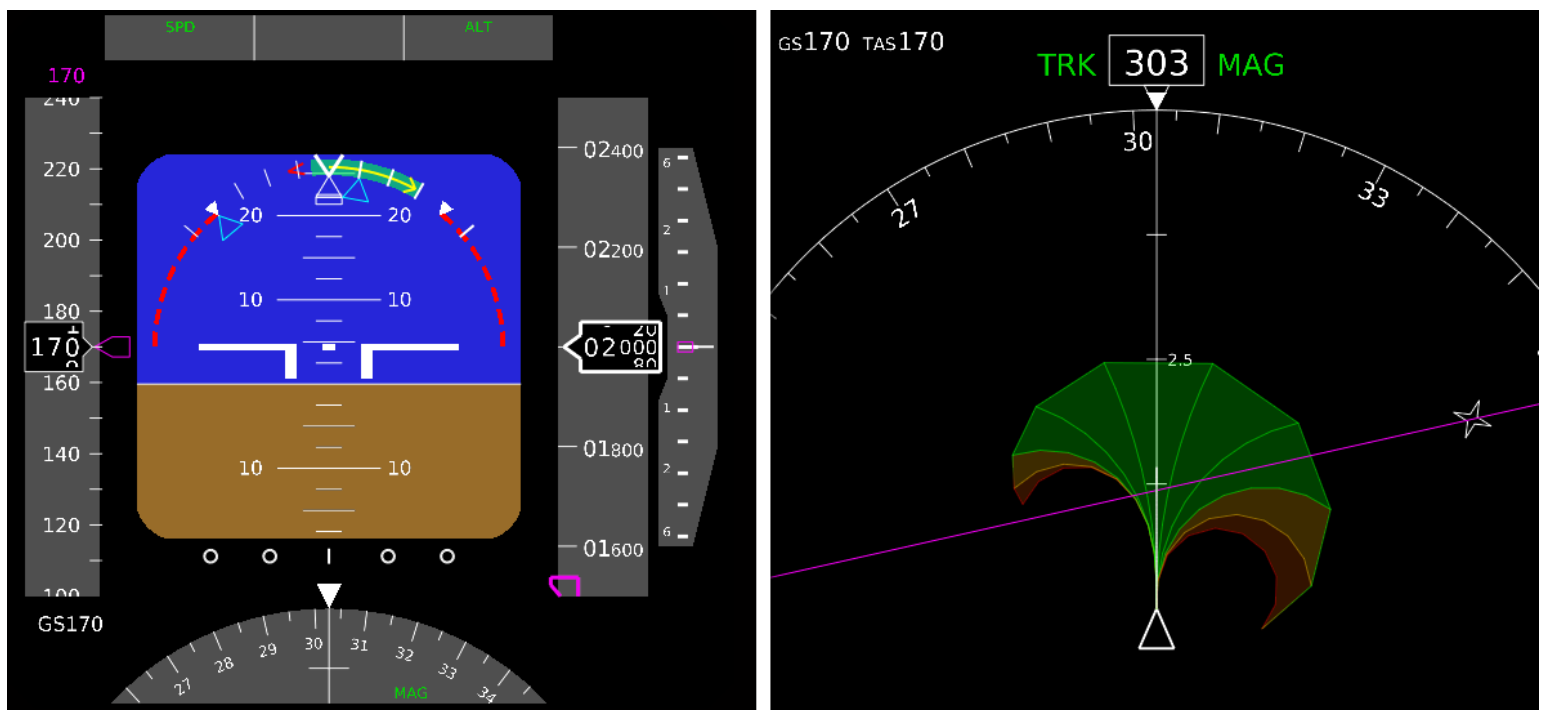

Figure F-1: Screen shots of the modified PFD and ND for explaining the additional interface elements 


\section{Appendix G}

\section{Questionnaire Results}

All subjects were asked to fill in a digital questionnaire at the end of the experiment. Below follow the results of the questionnaire. As one response failed to be recorded due to a technical error, $n=8$.

How would you assess your performance with respect to the interface in use?

Please select the most appropriate answer.

o My performance was constant regardless of the interface presented

o Especially the additional PFD information improved my performance

o Especially the additional ND information improved my performance

o Especially the additional PFD information reduced my performance

o Especially the additional ND information reduced my performance

All respondents answered "Especially the additional ND information reduced my performance".

Usefulness of features

Please rate the listed features in terms of how useful they were for the tasks you performed in the experiment.

"In control of the aircraft with the failure present, I relied on the information from the following feature..."

The attainable roll rate limits (the green bar on the PFD) 


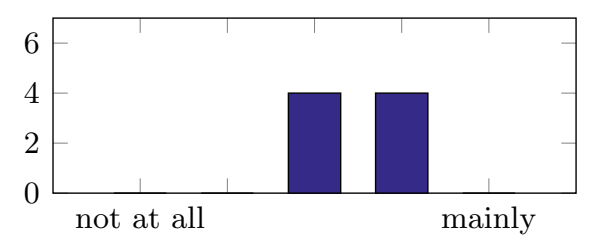

Figure G-1: The attainable roll rate limits

The current roll rate preview (the yellow arrow on the PFD)

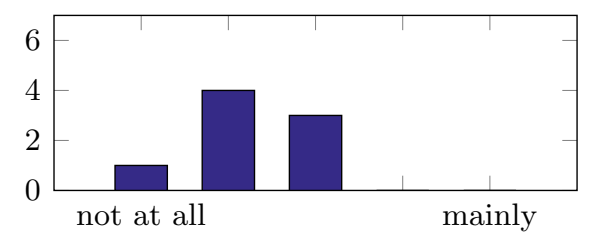

Figure G-2: The current roll rate preview

The bank angle preview (the blue triangles on the PFD)

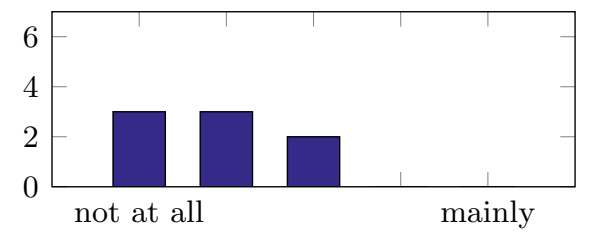

Figure G-3: The bank angle preview

The roll-out indicator (the red open triangle on the PFD)

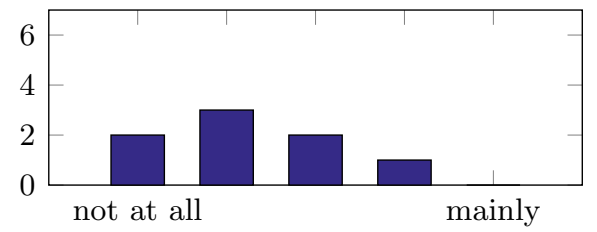

Figure G-4: The roll-out indicator

The navigation envelope (the fan-shaped element on the ND)

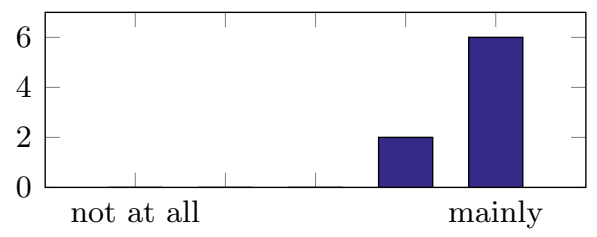

Figure G-5: The navigation envelope 
The trend vector (the white position preview element on the ND)

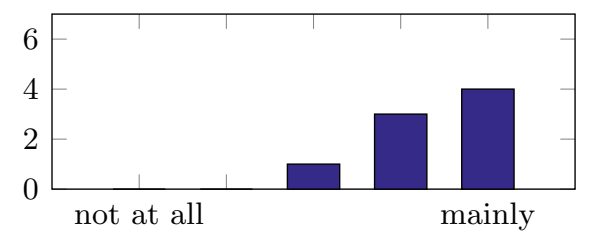

Figure G-6: The trend vector

Please describe to what extent you agree with the following statement.

The PFD modifications helped me in understanding the consequences of my control actions.

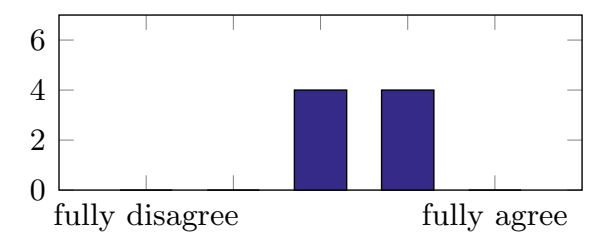

Figure G-7: The PFD helped in understanding consequences of actions

With respect to the baseline configuration, the PFD modifications made me more certain that the runway I selected was the optimal choice.

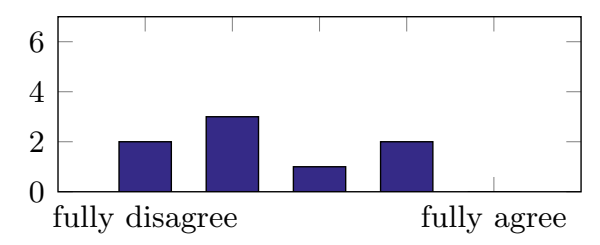

Figure G-8: The modified PFD clarified runway selection

The navigation envelope (on the ND) helped me in understanding the consequences of my control actions.

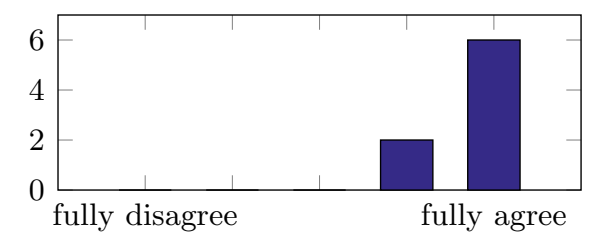

Figure G-9: The ND helped in understanding consequences of actions

With respect to the baseline configuration, the navigation envelope (on the ND) made me more certain that the runway I selected was the optimal choice. 


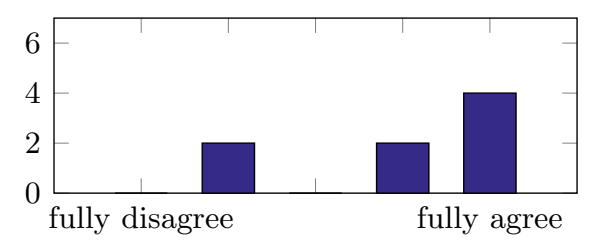

Figure G-10: The modified ND clarified runway selection

I feel that display additions like the ones presented would help me perform better during a performance-altering emergency.

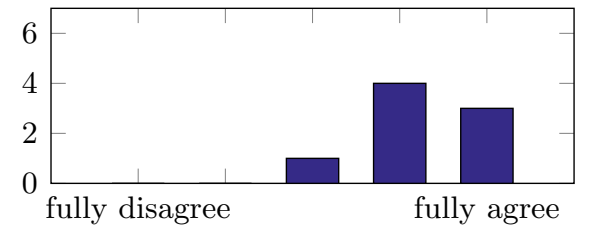

Figure G-11: Display additions like these are helpful

I feel that display additions like the ones presented would introduce too much clutter on the displays to be useful.

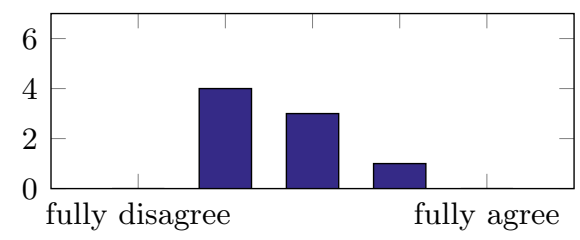

Figure G-12: Display additions like these introduce too much clutter

Did you feel you became more proficient in using the new interfaces during the experiment?

In other words, when the initial training was completed, do you think your performance still kept improving towards the end of the experiment?

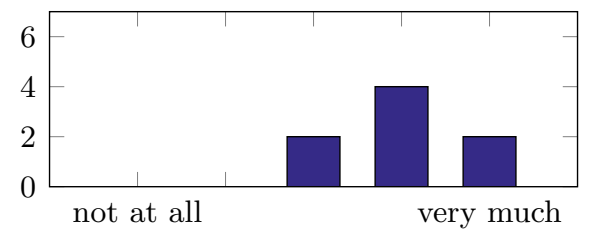

Figure G-13: Training effect during experiment 


\section{Appendix $\mathrm{H}$}

\section{Latin Square Distribution}

As there were two independent variables (FAIL and DISP) with two and three levels, respectively, six different experimental conditions existed. Nine pilots each completed one repetition, two different Latin square distributions were made: one for pilots one through six, and another one for pilots seven through nine. 


\begin{tabular}{|c|c|c|c|c|c|c|c|c|c|}
\hline Trial & \multicolumn{3}{|c|}{1} & \multicolumn{3}{|c|}{2} & \multicolumn{3}{|c|}{3} \\
\hline 1 & Moderate & Regular & Baseline & Severe & Regular & ND+ & Moderate & Mirrored & PFD+ \\
\hline 2 & Severe & Mirrored & PFD+ & Moderate & Regular & Baseline & Severe & Regular & $\mathrm{ND}+$ \\
\hline 3 & Moderate & Mirrored & $\mathrm{ND}+$ & Severe & Mirrored & PFD+ & Moderate & Regular & Baseline \\
\hline 4 & Severe & Regular & Baseline & Moderate & Mirrored & ND+ & Severe & Mirrored & PFD+ \\
\hline 5 & Moderate & Mirrored & PFD+ & Severe & Regular & Baseline & Moderate & Mirrored & ND+ \\
\hline 6 & Severe & Regular & $\mathrm{ND}+$ & Moderate & Mirrored & PFD+ & Severe & Regular & Baseline \\
\hline
\end{tabular}

\begin{tabular}{r||lll|lll|lll|}
\multicolumn{1}{|c|}{ Subject } \\
Trial & \multicolumn{2}{c|}{$\mathbf{4}$} & \multicolumn{3}{c|}{$\mathbf{5}$} & \multicolumn{2}{c|}{$\mathbf{6}$} \\
\hline 1 & Severe & Regular & Baseline & Moderate & Mirrored & ND+ & Severe & Mirrored & PFD+ \\
2 & Moderate & Mirrored & PFD+ & Severe & Regular & Baseline & Moderate & Mirrored & ND+ \\
3 & Severe & Regular & ND+ & Moderate & Mirrored & PFD+ & Severe & Regular & Baseline \\
4 & loderate & Regular & Baseline & Severe & Regular & ND+ & Moderate & Mirrored & PFD+ \\
5 & Severe & Mirrored & PFD+ & Moderate & Regular & Baseline & Severe & Regular & ND+ \\
6 & Moderate & Mirrored & ND+ & Severe & Mirrored & PFD+ & Moderate & Regular & Baseline \\
\hline
\end{tabular}

\begin{tabular}{|c|c|c|c|c|c|c|c|c|c|}
\hline Trial & & 7 & & & 8 & & & 9 & \\
\hline 1 & Moderate & Regular & PFD+ & Severe & Regular & Baseline & Moderate & Mirrored & ND+ \\
\hline 2 & Severe & Mirrored & $\mathrm{ND}+$ & Moderate & Regular & PFD+ & Severe & Regular & Baseline \\
\hline 3 & Moderate & Mirrored & Baseline & Severe & Mirrored & ND+ & Moderate & Regular & PFD+ \\
\hline 4 & Severe & Regular & PFD+ & Moderate & Mirrored & Baseline & Severe & Mirrored & ND+ \\
\hline 5 & Moderate & Mirrored & ND+ & Severe & Regular & PFD+ & Moderate & Mirrored & Baseline \\
\hline 6 & Severe & Regular & Baseline & Moderate & Mirrored & $\mathrm{ND}+$ & Severe & Regular & PFD+ \\
\hline
\end{tabular}

\section{Subject}

\begin{tabular}{|c|c|c|c|c|c|c|c|c|}
\hline \multicolumn{3}{|l|}{ Trial } & \multicolumn{3}{|c|}{11} & \multicolumn{3}{|c|}{12} \\
\hline 1 Severe & Regular & PFD+ & Moderate & Mirrored & Baseline & Severe & Mirrored & $\mathrm{ND}+$ \\
\hline 2Moderate & Mirrored & ND+ & Severe & Regular & PFD+ & Moderate & Mirrored & Baseline \\
\hline 3/Severe & Regular & Baseline & Moderate & Mirrored & ND+ & Severe & Regular & PFD+ \\
\hline 4 Moderate & Regular & PFD+ & Severe & Regular & Baseline & Moderate & Mirrored & ND+ \\
\hline 5 Severe & Mirrored & $\mathrm{ND}+$ & Moderate & Regular & PFD+ & Severe & Regular & Baseline \\
\hline 6 Moderate & Mirrored & Baseline & Severe & Mirrored & ND+ & Moderate & Regular & PFD+ \\
\hline
\end{tabular}

Figure H-1: Latin Square Distribution 


\section{Appendix I}

\section{Example Experiment Data Summary}

The sheet in this appendix shows how notes were taken during the experiment for one subject. For each trial, the log start time was recorded and the experimental condition was verified. The runway selection was noted together with the subject's description of the proposed navigation strategy. Additional notes were taken if potentially relevant. 


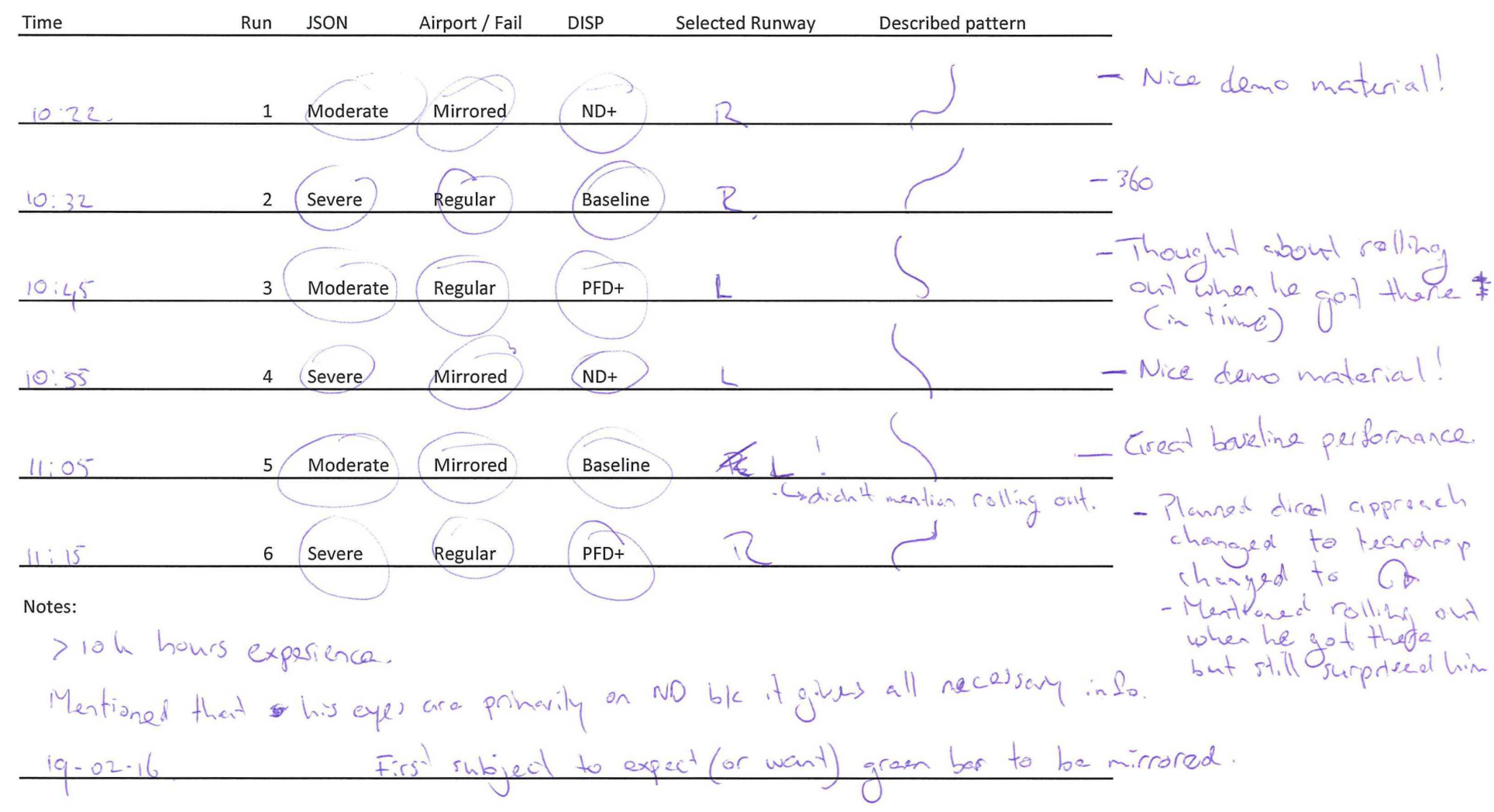


Appendix J Example TLX Sheet

The included file shows how the TLX sheets were expected to be filled in by the participants after every trial; hence, every participant filled in six TLX sheets. 


\begin{tabular}{|c|c|c|c|}
\hline \multicolumn{4}{|c|}{ NASA Task Load Index (TLX) rating sheet } \\
\hline \multicolumn{2}{|l|}{ Subject: 9} & \multicolumn{2}{|r|}{ Run: $\quad$ } \\
\hline \multicolumn{3}{|c|}{ Step 1 Sources of load } & Step 2 Magnitude of load \\
\hline & $\square$ Physical demand & $\begin{array}{l}\text { Mental demand } \\
\llcorner,|,|,|,|,|,||,|,|,|, \mid\end{array}$ \\
\hline \multicolumn{2}{|c|}{ Mental demand } & $\square$ Temporal demand & \multirow{3}{*}{$\begin{array}{l}\text { Low } \\
\text { Physical demand } \\
\begin{array}{l}\lfloor,|,|,|,|,|,|,|,|,|,| \\
\text { Low }\end{array}\end{array}$} \\
\hline \multicolumn{2}{|c|}{$\square$ Mental demand } & $\triangle$ Performance & \\
\hline \multicolumn{2}{|l|}{ Q Mental demand } & $\square$ Frustration level & \\
\hline \multirow{2}{*}{\multicolumn{2}{|c|}{$\begin{array}{l}\square \text { Physical demand } \\
\text { 四-Physical demand }\end{array}$}} & \multirow{2}{*}{$\begin{array}{l}\square \text { Temporal demand } \\
\square \text { Performance }\end{array}$} & \multirow{3}{*}{ 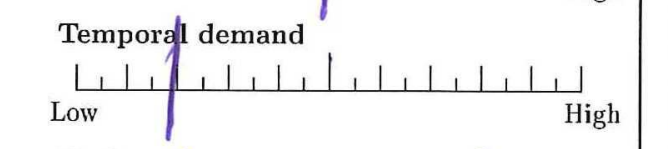 } \\
\hline & & & \\
\hline \multicolumn{2}{|c|}{$\begin{array}{l}\square \text { Physical demand } \\
\square \text { Physical demand }\end{array}$} & Effort & \\
\hline \multirow{2}{*}{\multicolumn{2}{|c|}{$\begin{array}{l}\text { 12. Temporal demand } \\
\square \text { Temporal demand } \\
\text { Temporal demand }\end{array}$}} & $\square$ Performance & $\left\llcorner, 1,||,,||,, \mid, 1, \frac{9}{4}, 1,1\right.$ \\
\hline & and & $\begin{array}{l}\text { ए Performance } \\
\text { ब Effort }\end{array}$ & Good $\mid$ \\
\hline \multirow{2}{*}{\multicolumn{2}{|c|}{$\begin{array}{l}\square \text { Performance } \\
\text { Performance }\end{array}$}} & 因 Effort & $\lfloor, 1,1,1,1,||,,||,, \mid, 1$ \\
\hline & & $\square$ Frustration level & \\
\hline \multicolumn{2}{|l|}{ 因-Effort } & $\square$ Frustration level & $\begin{array}{l}\text { Frustratipn level } \\
L,|,|,|,|,|,|,|,|, \mid \text { High }\end{array}$ \\
\hline \multicolumn{4}{|c|}{ NASA TLX rating descriptions } \\
\hline Title & Endpoints & \multicolumn{2}{|l|}{ s Description } \\
\hline Mental demand & Low, High & \multicolumn{2}{|c|}{$\begin{array}{l}\text { How much mental and perceptual activity was required? (e.g. thinking, deciding, } \\
\text { calculating, remembering, looking, searching, etc.) Was the task easy or demanding, } \\
\text { simple or complex, exacting or forgiving? }\end{array}$} \\
\hline Physical demand & Low, High & \multicolumn{2}{|c|}{$\begin{array}{l}\text { How much physical activity was required (e.g. pushing, pulling, turning, controlling, } \\
\text { activating, etc.)? Was the task easy or demanding, slow or brisk, slack or } \\
\text { strenuous, restful or laborous? }\end{array}$} \\
\hline Temporal demand & Low, High & $\begin{array}{l}\text { How much time pres: } \\
\text { task elements occure }\end{array}$ & $\begin{array}{l}\text { ou feel due to the rate or pace at which the tasks or } \\
\text { he pace slow and leisurely or rapid and frantic? }\end{array}$ \\
\hline Effort & Low, High & $\begin{array}{l}\text { How hard did you ha } \\
\text { of performance? }\end{array}$ & rk (mentally or physically) to accomplish your level \\
\hline Performance & Good, Poor & $\begin{array}{l}\text { How successful do yo } \\
\text { by the experimenter } \\
\text { performance in accon }\end{array}$ & $\begin{array}{l}\text { ou were in accomplishing the goals of the task set } \\
\text { elf)? How satisfied were you with your } \\
\text { these goals? }\end{array}$ \\
\hline Frustration Level & Low, High & $\begin{array}{l}\text { How insecure, discou } \\
\text { content, relaxed and }\end{array}$ & $\begin{array}{l}\text { itated, stressed and annoyed versus secure, gratified, } \\
\text { int did you feel during the task? }\end{array}$ \\
\hline
\end{tabular}




\section{Appendix K}

\section{Statistical Results}

This appendix presents the statistical data that was used to obtain the results listed in the accompanying paper. For discerning between statistical significance and non-significance, a $p$-value of 0.05 was used as threshold.

\section{K-1 Runway Selection}

The resultant data type from the runway selection was dichotomous, as the result was registered as either preferring a turn in the direction of the impaired turn performance or the nominal turn perforance. Therefore, Cochran's $Q$ test was used to compare for a significant difference between the runway selection as a function of the independent variable levels. A significant result was not found: $Q(5,9)=5.56, p=0.352)$, see Tables K-1 and K-2.

Table K-1: Frequencies

\begin{tabular}{lrr}
\hline & \multicolumn{2}{c}{ Value } \\
\hline & 0 (nominal) & 1 (impaired) \\
\hline M_Base & 3 & 6 \\
M_PFD & 2 & 7 \\
M_ND & 4 & 5 \\
S_Base & 2 & 7 \\
S_PFD & 1 & 8 \\
S_ND & 0 & 9 \\
\hline
\end{tabular}


Table K-2: Test Statistics

\begin{tabular}{lr}
\hline N & 9 \\
Cochran's Q & $5.556^{\mathrm{a}}$ \\
df & 5 \\
Asymp. Sig. & .352 \\
\hline
\end{tabular}

a. 1 is treated as a success.

\section{K-2 Intercept Strategy}

The resultant data type from the localizer intercept strategy was dichotomous, as the result was registered as either a direct or an indirect localizer intercept was performed. Therefore, Cochran's $Q$ test was used to compare for a significant difference between the runway selection as a function of the independent variable levels. A significant result was found: $Q(2,9)=$ $8.4, p=0.015)$, see Tables K-3 and K-4.

Table K-3: Frequencies

\begin{tabular}{lrr}
\hline & \multicolumn{2}{c}{ Value } \\
\hline & 0 (direct) & 1 (indirect) \\
\hline S_Base & 3 & 6 \\
S_PFD & 4 & 5 \\
S_ND & 8 & 1 \\
\hline
\end{tabular}

Table K-4: Test Statistics

\begin{tabular}{lr}
\hline N & 9 \\
Cochran's Q & $8.400^{\mathrm{a}}$ \\
df & 2 \\
Asymp. Sig. & .015 \\
\hline
\end{tabular}

a. 0 is treated as a success.

Results of the post-hoc analysis using McNemar's test are shown in Table K-5. The Bonferroni correction requires the $p$-values to be less than $0.05 / 3=0.0167$. Therefore, we can see that none of the pairwise comparisons yields a significant result.

Table K-5: Test Statistics (McNemar's Test)

\begin{tabular}{lrrr}
\hline & S_Base \& S_PFD & S_Base \& S_ND & S_PFD \& S_ND \\
\hline $\mathrm{N}$ & 9 & 9 & 9 \\
Exact Sig. (2-tailed) & $1.000^{\mathrm{b}}$ & $.063^{\mathrm{b}}$ & $.125^{\mathrm{b}}$ \\
\hline
\end{tabular}

b. Binomial distribution used. 


\section{K-3 Analysis of Work Load}

NASA TLX (Hart \& Staveland, 1988) forms were used to perform a self-assessment of the experienced work load during the different trials within the last phase (intercept) of the experiment. The resulting overall work load statistic is a weighted sum of different (ranked) categories $^{1}$, which is calculated using Equation K-1.

$$
\frac{1}{15} \sum_{i=1}^{6} w_{i} s_{i}
$$

In this equation, $w_{i}$ represents the weight for a category, and $s_{i}$ represents the score for that category. Each weight has a value from 0 and 5, and each score has a value from 0 to 100. The sum of all weights is 15, resulting in the final score being between 0 and 100 .

As the both the scores and their variance may vary from subject to subject, the resulting data set was treated as ordinal data. As a result, a regular ANOVA can not be used for the statistical analysis. Instead, Friedman's ANOVA was used, which expects the data to be ordinal instead. The test results are showin in Tables K-6 and K-7.

Table K-6: Ranks

\begin{tabular}{lr}
\hline & Mean Rank \\
\hline M_Base & 3.56 \\
M_PFD & 3.11 \\
M_ND & 2.39 \\
S_Base & 4.50 \\
S_PFD & 4.56 \\
S_ND & 2.89 \\
\hline
\end{tabular}

Table K-7: Test Statistics (Friedman)

\begin{tabular}{lr}
\hline $\mathrm{N}$ & 9 \\
Chi-Square & 10.000 \\
df & 5 \\
Asymp. Sig. & .075 \\
\hline
\end{tabular}

It can be seen that the different levels do not produce statistically significant results $\left(\chi^{2}(5)=\right.$ $10.00, p=0.075)$.

\section{K-4 Analysis of Safety Metrics}

For each of these metrics, two Friedman's ANOVAs were performed (one for each level of the FAIL independent variable). This makes it possible to perform fewer pairwise comparisons in a

\footnotetext{
${ }^{1}$ These categories are mental load, physical load, temporal demand, performance, effort, and frustration.
} 
post-hoc analysis, which allows us to make a smaller correction on the $p$-values, at the expense of not being able to detect a significant result between the different levels of FAIL. If the result was significant, Wilcoxon's Signed-Rank test was used to perform paired comparisons. The Bonferroni-corrected significance level is $0.05 / 3=0.0167$ for all comparisons.

\section{Completion Time (Table K-8 through K-13)}

This section provides the results of the statistical analysis of the time until glide slope capture. Table K-11 shows a significant influence of the display level for the severe case. Table K-13 reveals that rankings of Baseline and PFD+ levels are significantly different $(p<0.0167)$.

Table K-8: Ranks

\begin{tabular}{lr}
\hline & Mean Rank \\
\hline M_Base & 1.78 \\
M_PFD & 1.78 \\
M_ND & 2.44 \\
\hline
\end{tabular}

Table K-9: Test Statistics (Friedman)

\begin{tabular}{lr}
\hline $\mathrm{N}$ & 9 \\
Chi-Square & 2.667 \\
$\mathrm{df}$ & 2 \\
Asymp. Sig. & .264 \\
\hline
\end{tabular}

Table K-10: Ranks

\begin{tabular}{lr}
\hline & Mean Rank \\
\hline S_Base & 2.78 \\
S_PFD & 1.67 \\
S_ND & 1.56 \\
\hline
\end{tabular}

Table K-11: Test Statistics (Friedman)

\begin{tabular}{lr}
\hline $\mathrm{N}$ & 9 \\
Chi-Square & 8.222 \\
df & 2 \\
Asymp. Sig. & .016 \\
\hline
\end{tabular}


Table K-12: Ranks

\begin{tabular}{rrrrr}
\hline & N & Mean Rank & Sum of Ranks \\
\hline S_PFD - S_Base & Negative Ranks & 8 & 5.50 & 44.00 \\
& Positive Ranks & 1 & 1.00 & 1.00 \\
Ties & 0 & & \\
Total & 9 & & \\
S_ND - S_Base & Negative Ranks & 8 & 5.25 & 42.00 \\
& Positive Ranks & 1 & 3.00 & 3.00 \\
& Ties & 0 & & \\
Total & 9 & & 33.00 \\
S_ND - S_PFD & Negative Ranks & 5 & 6.60 & 12.00 \\
& Positive Ranks & 4 & 3.00 & \\
& Ties & 0 & & \\
& Total & 9 & & \\
\hline
\end{tabular}

Table K-13: Test Statistics (Wilcoxon Signed Ranks)

\begin{tabular}{lrrr}
\hline & S_PFD - S_Base & S_ND - S_Base & S_ND - S_PFD \\
\hline Z & -2.547 & -2.310 & -1.244 \\
Asymp. Sig. (2-tailed) & .011 & .021 & .214 \\
\hline
\end{tabular}

\section{Maximum Bank Angle (Table K-14 through K-19)}

This section provides the results of the statistical analysis of the maximum bank angle. Table K-17 shows a significant influence of the display level for the severe case. Table K-19 shows that at the corrected significance level $(p<0.0167)$, no rankings are significantly different.

Table K-14: Ranks

\begin{tabular}{lr}
\hline & Mean Rank \\
\hline M_Base & 2.11 \\
M_PFD & 1.56 \\
M_ND & 2.33 \\
\hline
\end{tabular}

Table K-15: Test Statistics (Friedman)

\begin{tabular}{lr} 
N & 9 \\
Chi-Square & 2.889 \\
df & 2 \\
Asymp. Sig. & .236 \\
\hline
\end{tabular}

Table K-16: Ranks

\begin{tabular}{lr}
\hline & Mean Rank \\
\hline S_Base & 2.56 \\
S_PFD & 2.11 \\
S_ND & 1.33 \\
\hline
\end{tabular}

Table K-17: Test Statistics (Friedman)

\begin{tabular}{lr}
\hline N & 9 \\
Chi-Square & 6.889 \\
df & 2 \\
Asymp. Sig. & .032 \\
\hline
\end{tabular}


Table K-18: Ranks

\begin{tabular}{rrrrr}
\hline & N & Mean Rank & Sum of Ranks \\
\hline S_PFD - S_Base & Negative Ranks & 7 & 5.71 & 40.00 \\
& Positive Ranks & 2 & 2.50 & 5.00 \\
Ties & 0 & & \\
Total & 9 & & \\
S_ND - S_Base & Negative Ranks & 7 & 6.00 & 42.00 \\
& Positive Ranks & 2 & 1.50 & 3.00 \\
& Ties & 0 & & \\
Total & 9 & & \\
S_ND - S_PFD & Negative Ranks & 8 & 5.00 & 50.00 \\
& Positive Ranks & 1 & 5.00 & 5.00 \\
& Ties & 0 & & \\
& Total & 9 & & \\
\hline
\end{tabular}

Table K-19: Test Statistics (Wilcoxon Signed Ranks)

\begin{tabular}{lrrr}
\hline & S_PFD - S_Base & S_ND - S_Base & S_ND - S_PFD \\
\hline Z & -2.073 & -2.310 & -2.073 \\
Asymp. Sig. (2-tailed) & .038 & .021 & .038 \\
\hline
\end{tabular}

\section{Maximum Roll Rate (Table K-20 through K-23)}

No significant effect was found for this metric.

Table K-20: Ranks

\begin{tabular}{lr}
\hline & Mean Rank \\
\hline M_Base & 2.44 \\
M_PFD & 1.67 \\
M_ND & 1.89 \\
\hline
\end{tabular}

Table K-21: Test Statistics (Friedman)

\begin{tabular}{lr} 
N & 9 \\
Chi-Square & 2.889 \\
df & 2 \\
Asymp. Sig. & .236 \\
\hline
\end{tabular}

Table K-22: Ranks

\begin{tabular}{lr}
\hline & Mean Rank \\
\hline S_Base & 2.44 \\
S_PFD & 1.89 \\
S_ND & 1.67 \\
\hline
\end{tabular}

Table K-23: Test Statistics (Friedman)

\begin{tabular}{lr}
\hline N & 9 \\
Chi-Square & 2.889 \\
df & 2 \\
Asymp. Sig. & .236 \\
\hline
\end{tabular}




\section{K-5 Analysis of Performance Metrics}

For each of these metrics, two Friedman's ANOVAs were performed (one for each level of the FAIL independent variable). This makes it possible to perform fewer pairwise comparisons in a post-hoc analysis, which allows us to make a smaller correction on the $p$-values, at the expense of not being able to detect a significant result between the different levels of FAIL. If the result was significant, Wilcoxon's Signed-Rank test was used to perform paired comparisons. The Bonferroni-corrected significance level is $0.05 / 3=0.0167$ for all comparisons.

\section{Lateral Position Error at Glide Slope (Table K-24 through K-27)}

No significant effect was found for this metric.

Table K-24: Ranks

\begin{tabular}{lr}
\hline & Mean Rank \\
\hline M_Base & 2.33 \\
M_PFD & 1.89 \\
M_ND & 1.78 \\
\hline
\end{tabular}

Table K-25: Test Statistics (Friedman)

\begin{tabular}{lr}
\hline $\mathrm{N}$ & 9 \\
Chi-Square & 1.556 \\
$\mathrm{df}$ & 2 \\
Asymp. Sig. & .459 \\
\hline
\end{tabular}

Table K-26: Ranks

\begin{tabular}{lr}
\hline & Mean Rank \\
\hline S_Base & 1.89 \\
S_PFD & 2.11 \\
S_ND & 2.00 \\
\hline
\end{tabular}

Table K-27: Test Statistics (Friedman)

\begin{tabular}{lr}
\hline $\mathrm{N}$ & 9 \\
Chi-Square & .222 \\
$\mathrm{df}$ & 2 \\
Asymp. Sig. & .895 \\
\hline
\end{tabular}

Heading Error at Glide Slope (Table K-28 through K-31)

No significant effect was found for this metric. 
Table K-28: Ranks

\begin{tabular}{lr}
\hline & Mean Rank \\
\hline M_Base & 2.22 \\
M_PFD & 1.67 \\
M_ND & 2.11 \\
\hline
\end{tabular}

Table K-29: Test Statistics (Friedman)

\begin{tabular}{lr}
\hline $\mathrm{N}$ & 9 \\
Chi-Square & 1.556 \\
$\mathrm{df}$ & 2 \\
Asymp. Sig. & .459 \\
\hline
\end{tabular}

Table K-30: Ranks

\begin{tabular}{lr}
\hline & Mean Rank \\
\hline S_Base & 2.11 \\
S_PFD & 2.11 \\
S_ND & 1.78 \\
\hline
\end{tabular}

Table K-31: Test Statistics (Friedman)

\begin{tabular}{lr}
\hline N & 9 \\
Chi-Square & .667 \\
df & 2 \\
Asymp. Sig. & .717 \\
\hline
\end{tabular}

\section{Yaw Rate at Glide Slope (Table K-32 through K-35)}

No significant effect was found for this metric.

Table K-32: Ranks

\begin{tabular}{lr}
\hline & Mean Rank \\
\hline M_Base & 1.67 \\
M_PFD & 2.33 \\
M_ND & 2.00 \\
\hline
\end{tabular}

Table K-33: Test Statistics (Friedman)

\begin{tabular}{lr}
\hline $\mathrm{N}$ & 9 \\
Chi-Square & 2.000 \\
df & 2 \\
Asymp. Sig. & .368 \\
\hline
\end{tabular}

Table K-34: Ranks

\begin{tabular}{lr}
\hline & Mean Rank \\
\hline S_Base & 1.78 \\
S_PFD & 2.22 \\
S_ND & 2.00 \\
\hline
\end{tabular}

Table K-35: Test Statistics (Friedman)

\begin{tabular}{lr}
\hline N & 9 \\
Chi-Square & .889 \\
df & 2 \\
Asymp. Sig. & .641 \\
\hline
\end{tabular}




\section{References}

Anderson, J. D. (1999). Aircraft Performance and Design. Boston: McGraw-Hill. Available from http://www.sciencedirect.com/science/article/pii/B0122274105009121

Antoniewicz, R. F., Duke, E. L., \& Patterson, B. B. (1988). User's manual for Interactive LINEAR, a FORTRAN program to derive linear aircraft models. Edwards, CA: NASA, Scientific and Technical Information Division.

Atkins, E. M., Portillo, I. A., \& Strube, M. J. (2006). Emergency Flight Planning Applied to Total Loss of Thrust. Journal of Aircraft, 43(4), 1205-1216.

Bisantz, a. M., \& Vicente, K. J. (1994). Making the abstraction hierarchy concrete (Vol. 40).

Borst, C., Mulder, M., \& Van Paassen, M. M. (2010). Design and Simulator Evaluation of an Ecological Synthetic Vision Display. Journal of Guidance, Control, and Dynamics, 33(5), 1577-1591.

Borst, C., Sjer, F. A., Mulder, M., Van Paassen, M. M., \& Mulder, J. A. (2008). Ecological Approach to Support Pilot Terrain Awareness After Total Engine Failure. Journal of Aircraft, 45(1), 159-171.

Chen, T. L., \& Pritchett, A. R. (2001). Development and Evaluation of a Cockpit Decision-Aid for Emergency Trajectory Generation. Journal of Aircraft, 38(5), 935-943.

Comerford, D., Brandt, S. L., \& Mogford, R. (2013). NASA / CP - 2013 - 216513 NASA's Single -Pilot Operations Technical Interchange Meeting : Proceedings and Findings (Tech. Rep. No. April). Moffett Field: NASA.

Dam, S. B. J. van, Mulder, M., \& Paassen, M. M. van. (2008). Ecological Interface Design of a Tactical Airborne Separation Assistance Tool. Ieee Transactions on Systems Man and Cybernetics Part a-Systems and Humans, 38(6), 1221-1233.

Edwards, C., Lombaerts, T., \& Smaili, H. (2010). Fault tolerant flight control. Lecture Notes in Control and Information Sciences, 399.

Hart, S. G., \& Staveland, L. E. (1988). Development of NASA-TLX (Task Load Index): Results of Empirical and Theoretical Research. Advances in Psychology, 52(C), 139-183.

Linden, C. A. A. M. van der. (1996). DASMAT-Delft University Simulation Model and Analysis Tool. Delft: Delft University of Technology. 
Lombaerts, T., Schuet, S., Wheeler, K., Acosta, D., \& Kaneshige, J. (2013). Robust maneuvering envelope estimation based on reachability analysis in an optimal control formulation. 2013 2nd International Conference on Control and Fault-Tolerant Systems (Systol), 318-323.

Maciejowski, J. M., \& Jones, C. N. (n.d.). MPC Fault-tolerant Flight Control Case Study: Flight 1862. Cambridge. Available from http://www-control.eng.cam.ac.uk/Homepage/papers/cued_control_7.pdf

Mercado, G. A., Mulder, M., \& Paassen, M. M. van. (2010). Analysis of Air Traffic Controller Workload Reduction Based on the Solution Space for the Merging Task. In Aiaa guidance, navigation, and control conference.

Meuleau, N., Neukom, C., Plaunt, C., Smith, D. E., \& Smith, T. (2011). The Emergency Landing Planner Experiment. In Icaps-11 (pp. 60-67).

Meuleau, N., Plaunt, C., Smith, D. E., \& Smith, T. (2009). An Emergency Landing Planner for Damaged Aircraft. In Proceedings of the twenty first innovative applications of artificial intelligence conference (Vol. 21, pp. 114-121). AIAA Press.

Naikar, N. (2011). Cognitive Work Analysis : Foundations, Extensions, and Challenges. (1986).

Naikar, N., Hopcroft, R., \& Moylan, A. (2005). Work Domain Analysis : Theoretical Concepts and Methodology. Available from http://www.dtic.mil/dtic/tr/fulltext/u2/a449707.pdf

Netherlands Aviation Safety Board. (1992). Aircraft Accident Report - El Al Flight 1862.

Oort, E. R. van, Chu, Q. P., \& Mulder, J. A. (2011). Maneuver Envelope Determination through Reachability Analysis. In Advances in aerospace guidance, navigation and control (pp. 91-102). Berlin: Springer Berlin Heidelberg. Available from http://link. springer.com/chapter/10.1007/978-3-642-19817-5_8

Rasmussen, J. (1983). Skills, rules, and knowledge; signals, signs, and symbols, and other distinctions in human performance models. IEEE Transactions on Systems, Man, and Cybernetics, SMC-13(3), 257-266.

Rasmussen, J., \& Vicente, K. K. J. (1989). Coping with Human Errors through System-Design - Implications for Ecological Interface Design. International Journal of Man-Machine Studies, 31(5), 517-534.

Ruijgrok, G. J. J. (2009). Elements of Airplane Performance. Delft University Press.

Schutte, P. C., Goodrich, K. H., Cox, D. E., Jackson, E. B., Palmer, M. T., Pope, A. T., et al. (2007). The Naturalistic Flight Deck System: An Integrated System Concept for Improved Single-Pilot Operations (Tech. Rep. No. December). Hampton, Virginia: NASA Langley Research Center.

Smaili, H., Breeman, J., Lombaerts, T., \& Stroosma, O. (2011). A benchmark for fault tolerant flight control evaluation. In 4 th european conference for aerospace systems (eucass). Available from http://www.eucass-proceedings.eu/10.1051/eucass/201306333

Smaili, M., \& Mulder, J. (2003). Flight data reconstruction and simulation of the 1992 Amsterdam Bijlmermeer airplane accident. Modeling and Simulation Technologies Conference(August). Available from http://arc.aiaa.org/doi/abs/10.2514/6.2000-4586

Smaili, M. H., Breeman, J., Lombaerts, T. J. J., \& Joosten, D. A. (2006). A simulation benchmark for integrated fault tolerant flight control evaluation. In Collection of 
technical papers - aiaa modeling and simulation technologies conference, 2006 (Vol. 1, pp. 563-585).

Strube, M. J. (2005). Post-failure trajectory planning from feasible trim state sequences (Vol. 1431397). Unpublished doctoral dissertation. Available from http://ezproxy.net.ucf.edu/login?url=http://search.proquest.com/docview/ 304994997?accountid=10003\$\delimiter"026E30F\$nhttp://sfx.fcla.edu/ ucf?url_ver=Z39.88-2004\&rft_val_fmt=info: of i/fmt:kev: $\mathrm{mtx}$ :

dissertation\&genre=dissertations+\&+theses\&sid=ProQ:

ProQuest+Dissertations+\&+The

Tang, L., Roemer, M., Ge, J., Crassidis, A., Prasad, J., \& Belcastro, C. (2009). Methodologies for Adaptive Flight Envelope Estimation and Protection. In Aiaa guidance, navigation, and control conference (pp. 1-14).

Vicente, K. J., \& Rasmussen, J. (1992). Ecological interface design: Theoretical foundations. IEEE Transactions on Systems, Man and Cybernetics, 22(4), 589-606. Available from http://ieeexplore. ieee.org/xpls/abs_all.jsp?arnumber $=156574$

Watts, R., Claus Christmann, H., Johnson, E., Feigh, K., \& Tsiotras, P. (2012).

Development and evaluation of an automated path planning aid. Journal of Aircraft, $49(6), 1774-1785$. 